\title{
On Two-Phase Flow Solvers in Irregular Domains with Contact Line
}

\author{
Mathieu Lepilliez ${ }^{\mathrm{a}, \mathrm{b}, \mathrm{c},}$, Elena Roxana Popescu ${ }^{\mathrm{a}}$, Frederic Gibou ${ }^{\mathrm{d}, \mathrm{e}}$, Sébastien Tanguy ${ }^{\mathrm{a}}$ \\ ${ }^{a}$ Institut de Mécanique des Fluides de Toulouse, 2bis allée du Professeur Camille Soula, 31400 Toulouse, France \\ ${ }^{b}$ Centre National d'Etudes Spatiales, 18 Avenue Edouard Belin, 31401 Toulouse Cedex 9, France \\ ${ }^{c}$ Airbus Défence \& Space, 31 Avenue des Cosmonautes, 31402 Toulouse Cedex 4, France \\ ${ }^{d}$ Department of Mechanical Engineering, University of California, Santa Barbara, CA 93106-5070, United States \\ ${ }^{e}$ Department of Computer Science, University of California, Santa Barbara, CA 93106-5110, United States
}

\begin{abstract}
We present numerical methods that enable the direct numerical simulation of two-phase flows in irregular domains. A method is presented to account for surface tension effects in a mesh cell containing a triple line between the liquid, gas and solid phases. Our numerical method is based on the level-set method to capture the liquid-gas interface and on the single-phase Navier-Stokes solver in irregular domain proposed in [34] to impose the solid boundary in an Eulerian framework. We also present a strategy for the implicit treatment of the viscous term and how to impose both a Neumann boundary condition and a jump condition when solving for the pressure field. Special care is given on how to take into account the contact angle, the no-slip boundary condition for the velocity field and the volume forces. Finally, we present numerical results in two and three spatial dimensions evaluating our simulations with several benchmarks.
\end{abstract}

Keywords: Sharp Interface Methods, Irregular domains, Implicit Viscosity, Contact lines, Level Set Method, Ghost-Fluid Method

\section{Introduction}

Multiphase flows are ubiquitous in most modern processing technology and are critical to the understanding of a wide range of physical and biological phenomena. Consequently, the design of numerical methods enabling the simulation of multiphase flows is the subject of intense research. When considering solid boundaries, explicit descriptions of the geometry have demonstrated results of superior accuracy in the case of one-phase flows, when compared to implicit representations of the geometry. Indeed, body-fitted grids can be constructed to enable the accurate treatment of boundary conditions and to capture the rapid variations of the solution near walls, e.g. the turbulent boundary layer in high speed flows. In the case of multiphase flows, however, body-fitted approaches are less practical since conforming mesh must be reconstructed several time during the course of a simulation. This is, for example, the case of the simulation of bubbly flows or sprays, where the fluid interface can be strongly deformed and stretched. The automatic meshing necessary in such cases is a difficult task and in turn, the resulting mesh could lack the quality needed to guarantee accurate numerical results.

Email address: mathieu.lepilliez@imft.fr (Mathieu Lepilliez)

Preprint submitted to Journal of Computational Physics

March 18, 2016

(C) 2016. This manuscript version is made available under the Elsevier user license

http://www.elsevier.com/open-access/userlicense/1.0/ 
Since the pioneer work of Peskin [37], introducing the immersed boundary method, an important research effort has been devoted to the development of Eulerian fluid solvers in irregular domains. These methods have the advantage that no remeshing is necessary during the course of a simulation in the case of uniform grids. If adaptive grids are employed, then the remeshing is straightforward since the free boundary is embedded in the grid. Two classes of methods have emerged in the Eulerian framework: 'delta' formulations, where the Dirac distribution representing surface forces is approximated by a regularized function [52] (and the references therein) and 'sharp' approaches, where jump conditions at the free boundary are enforced at the discrete level $[15,19,39,50,51$, $62,64]$ (and the references therein). Formulations based on regularized Dirac distributions provide a pathway to easily extend single-phase to multiphase solvers $[8,31]$. On the other hand, these methods suffer from drawbacks that limit their use in some applications due to the inherent smearing of the solution near interfaces. Although efficient adaptive mesh refinement techniques $[2,20]$ can help reduce the extent of the smearing by imposing increased resolution where needed, 'sharp' numerical methods are still desired in some applications. These methods, however, are difficult to design due to the difficulty of imposing boundary conditions implicitly.

While several works have been presented in the last decade on immersed boundary methods for incompressible one-phase flows $[4,24,30,32,33,34,54,55,57,63]$, only a few $[28,35,56]$ have been dedicated to incompressible two-phase flows, especially in the case where a contact line of the liquid-gas interface is formed on the embedded solid boundary. In such a situation, two different interfaces must be managed in a computational cell, and the numerical solver for the pressure must impose both a jump condition between the liquid and the gas to account for surface tension effects and a Neumann boundary condition between the fluid and the solid phase to impose the no-slip boundary condition.

An attractive immersed boundary method for one-phase flows, based on a finite volume discretization of the Laplace operator, has been proposed in [34]. It enables to impose solid boundary conditions on the pressure and on the velocity with a second-order spatial accuracy and to maintain the symmetric definite positiveness of the resulting linear system. Consequently, standard methods such as the preconditioned conjugate gradient can be used to invert efficiently the linear system.

Contribution and outline of the paper: we extend the work of [34] to the more complex situation of two-phase flows. Indeed, to our knowledge, only a few methods are dedicated to the numerical simulation of multiphase flows in irregular domains. As two-phase flows are involved, surface tension has to be accounted for, even in the case where solid, liquid and gas are present in the same computational cell. To that extent, we have developed a new numerical scheme to account for both a pressure jump condition due to the surface tension and a Neumann condition on the solid domain in the same cells, as described in section 3.2. This spatial discretization enables the treatment of volume forces inside the mesh cells that are crossed by the contact line. In section 3.3, specific details are given on how to perform an implicit temporal discretization of the viscous terms with a coupled linear system involving all the velocity components, while imposing the jump condition on viscosity. In section 3.4, we describe how to enforce the Dirichlet boundary condition in computational cells crossed by the solid boundary, in order to ensure the no-slip boundary condition. To our knowledge, solving such a coupled system with an immersed boundary condition has never been addressed in the existing literature. Finally, we discuss in section 3.5 how to impose the appropriate contact angle by extrapolating the liquid-gas level-set function inside the solid domain with an iterative PDE. .

Several benchmarks are proposed in section 4. In the first part of section 4, one-phase flow 
simulations are presented in order to compare two possible approaches to compute the viscous terms in two and three spatial dimensions. Next, test-cases involving two-phase flows in a complex geometry (droplet deposited on a slanted wall, half-filled rotating spherical tank) are presented and compared to theoretical solutions in order to highlight the behavior of the proposed numerical methods for the computation of the surface tension and the volume forces in the grid cells that are both crossed by the liquid-gas interface and the solid boundary. Since orders of accuracy are difficult to determine for such simulations, grid sensitivity studies are presented for all the benchmarks in order to ascertain that all the computations converge to the correct solution.

\section{Equations and Standard Projection Methods}

The Navier-Stokes equations describe the motion of fluids at the continuum level. However, their formulations and approximations depend on how surface forces are represented. Nevertheless, standard state-of-the-art numerical approximations are based on the projection method for singlephase flows, introduced by Chorin [11].

\subsection{Single-Phase Flows}

Consider a domain $\Omega=\Omega_{l} \cup \Omega_{s}$ with boundary $\partial \Omega$. The regions $\Omega_{l}$ and $\Omega_{s}$ represent the fluid and solid regions, respectively. The boundary between the fluid and the solid is denoted by $\Gamma_{s}$. The incompressible Navier-Stokes equations for one-phase Newtonian flows are written as:

$$
\begin{gathered}
\nabla \cdot \mathbf{u}=0 \quad \text { in } \Omega_{l}, \\
\rho\left(\frac{\partial \mathbf{u}}{\partial t}+(\mathbf{u} \cdot \nabla) \mathbf{u}\right)=-\nabla p+\mu \Delta \mathbf{u}+\rho \mathbf{g} \quad \text { in } \Omega_{l},
\end{gathered}
$$

where $t$ the is time, $\rho$ the fluid density, $\mathbf{u}=(u, v, w)$ the velocity field, $\mu$ the viscosity assumed constant, $p$ the pressure and $\mathbf{g}$ the acceleration due to gravity.

In the case of single-phase flows, Chorin used the Hodge decomposition of vector fields, to design a three-stage projection method to solve the Navier-Stokes equations [11]: first, given a velocity $\mathbf{u}^{n}$ at time $t^{n}=n \Delta t$, an intermediate velocity $\mathbf{u}^{*}$ can be computed for a time step $\Delta t$ without considering the pressure component:

$$
\mathbf{u}^{*}=\mathbf{u}^{\mathbf{n}}-\Delta t\left(\left(\mathbf{u}^{\mathbf{n}} \cdot \nabla\right) \mathbf{u}^{\mathbf{n}}-\frac{\mu \Delta \mathbf{u}^{\mathbf{n}}}{\rho}-\mathbf{g}\right) .
$$

Second, the pressure field $p^{n+1}$ serves as the scalar function of the Hodge decomposition, satisfying:

$$
\nabla \cdot\left(\frac{\nabla p^{n+1}}{\rho}\right)=\frac{\nabla \cdot \mathbf{u}^{*}}{\Delta t}
$$

with homogeneous Neumann boundary conditions on $\partial \Omega$ and non-homogeneous Neumann boundary condition on $\Gamma_{s}$ :

$$
\left.\mathbf{n}_{s} \cdot \frac{\nabla p}{\rho}\right|_{\Gamma_{s}}=\left.\mathbf{n}_{s} \cdot\left(\mathbf{u}^{*}-\mathbf{u}_{s}\right)\right|_{\Gamma_{s}} .
$$

where $\mathbf{u}_{s}$ is the specified velocity field on the solid's boundary. Finally, the fluid velocity $\mathbf{u}^{n+1}$ is defined at the new time step $t^{n+1}$ as the projection of $\mathbf{u}^{*}$ onto the divergence-free space:

$$
\mathbf{u}^{n+1}=\mathbf{u}^{*}-\Delta t \frac{\nabla p^{n+1}}{\rho} .
$$




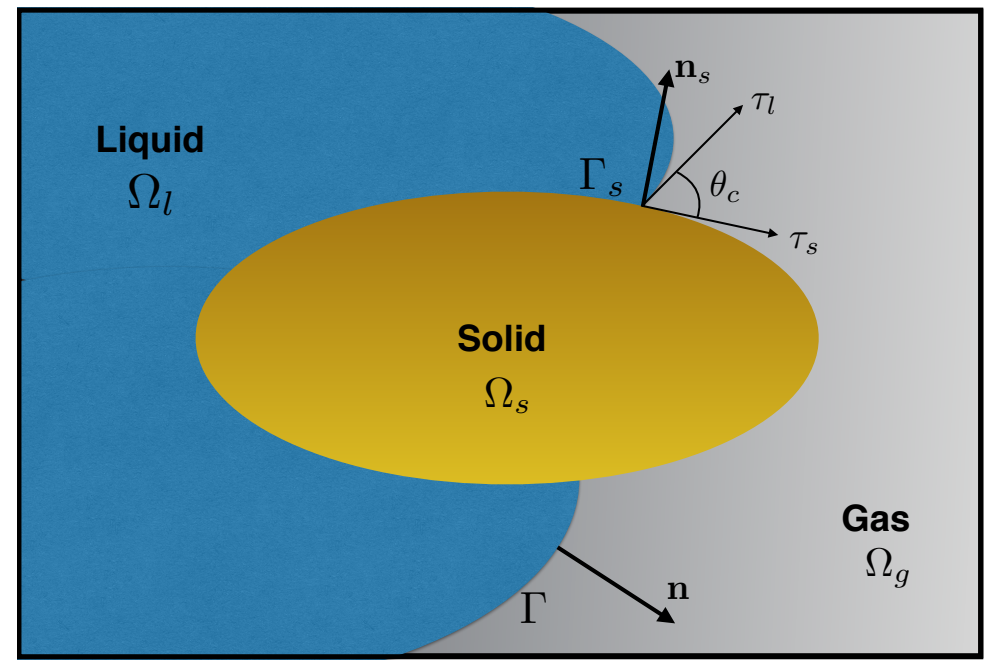

Figure 1: The computational domain in the case of two-phase flows in irregular domains.

Consider a computational domain, $\Omega$, that contains solid, liquid and gas regions denoted by $\Omega_{s}, \Omega_{l}$ and $\Omega_{g}$, respectively ${ }^{1}$ (see figure 1 ). We call $\Gamma$ the liquid's boundary and $\mathbf{n}$ its outward normal. Likewise, we call $\Gamma_{s}$ the solid's boundary and $\mathbf{n}_{s}$ its outward normal. Finally, $\partial \Omega$ denotes the boundary of $\Omega$. The incompressible Navier-Stokes equations for Newtonian two-phase flows can be described differently, whether the jump conditions are considered as functions in the entire computational domain, or as sharp jump conditions locally applied to the density, viscosity and pressure field. In what follows, we briefly present the main formulations that are used.

\subsubsection{The "Delta" Formulation}

The "delta" formulation $[42,47]$ writes the Navier-Stokes equations for two-phase flows as:

$$
\begin{gathered}
\nabla \cdot \mathbf{u}=0 \\
\rho\left(\frac{\partial \mathbf{u}}{\partial t}+(\mathbf{u} \cdot \nabla) \mathbf{u}\right)=-\nabla p+\rho \mathbf{g}+\nabla \cdot(2 \mu \underline{\underline{\mathbf{D}}})+\sigma \kappa \mathbf{n} \delta_{\Gamma},
\end{gathered}
$$

where $\sigma$ is the surface tension, $\kappa$ is the interface local mean curvature, $\delta_{\Gamma}$ a multidimensional Dirac distribution localized at the interface, and $\underline{\underline{\mathbf{D}}}$ the rate-of-deformation tensor defined as:

$$
\underline{\underline{D}}=\frac{\nabla \mathbf{u}+\nabla \mathbf{u}^{T}}{2}
$$

The density and the viscosity are piecewise constant and only vary across the interface. They can then be defined as:

\footnotetext{
${ }^{1}$ We consider a liquid region and a gas region for the sake of presentation, but this work is equally applicable to two liquids or two gases.
} 


$$
\begin{aligned}
& \rho=\rho_{l}+H_{\Gamma}\left(\rho_{g}-\rho_{l}\right), \\
& \mu=\mu_{l}+H_{\Gamma}\left(\mu_{g}-\mu_{l}\right),
\end{aligned}
$$

with $H_{\Gamma}$ denoting the Heaviside distribution, equal to 1 in the liquid and 0 in the gas phase, with the following definition for density and viscosity jump conditions:

$$
\begin{aligned}
& {[\rho]=\rho_{l}-\rho_{g},} \\
& {[\mu]=\mu_{l}-\mu_{g} .}
\end{aligned}
$$

When applying a projection method to the "delta" formulation, one obtains the following explicit discretization: first, solve for an intermediate velocity field $\mathbf{u}_{1}^{*}$ with

$$
\mathbf{u}_{1}^{*}=\mathbf{u}^{n}-\Delta t\left(\left(\mathbf{u}^{n} \cdot \nabla\right) \mathbf{u}^{n}-\frac{\nabla \cdot\left(2 \mu^{n+1} \underline{\underline{D}}^{n}\right)}{\rho^{n+1}}-\frac{\sigma \kappa^{n+1}}{\rho^{n+1}} \mathbf{n} \delta_{\Gamma}-\mathbf{g}\right) .
$$

We omit the subscripts on $\kappa$ and $\mu$, which are always estimated at $t^{n+1}$. Second, solve a Poisson equation to determine the Hodge field:

$$
\nabla \cdot\left(\frac{\nabla p^{n+1}}{\rho^{n+1}}\right)=\frac{\nabla \cdot \mathbf{u}_{1}^{*}}{\Delta t} .
$$

And finally project $\mathbf{u}_{1}^{*}$ onto the divergence-free velocity field:

$$
\mathbf{u}^{n+1}=\mathbf{u}_{1}^{*}-\Delta t \frac{\nabla p^{n+1}}{\rho^{n+1}} .
$$

This numerical scheme is usually used in the framework of the Continuum Surface Force (CSF) model, which requires the definition of smoothed functions $\delta_{\epsilon}$ and $H_{\epsilon}$ to approximate the Dirac and Heaviside distributions. The ease of implementation of this method comes with drawback of artificially thickening the interface. Unlike the CSF model, the "sharp" interface approach $[23,38,48]$ builds a sharp discretization of singular source terms, avoiding the introduction of a fictitious interface thickness. Some details on the theoretical equivalence between the jump condition formulation and the "delta" formulation is detailed in [25, 26].

\subsubsection{The Ghost-Fluid Primitive Viscous Method}

A splitting on the viscous-stress tensor can be applied :

$$
\begin{aligned}
\nabla \cdot(2 \mu \underline{\underline{\mathbf{D}}}) & =2 \mu \nabla \cdot(\underline{\underline{\mathbf{D}}})+2[\mu] \frac{\partial u_{n}}{\partial n} \delta_{\Gamma} \mathbf{n} \\
& =\mu \Delta \mathbf{u}+2[\mu]\left(\mathbf{n}^{T} \cdot \nabla \mathbf{u} \cdot \mathbf{n}\right) \delta_{\Gamma} \mathbf{n} .
\end{aligned}
$$

This leads to a new possible description of the intermediate velocity, where a jump condition in the viscous term has to be imposed. The reader may find a detailed demonstration of equation (2) in Appendix A.

In [23], the authors proposed a sharp interface method for incompressible two-phase flows based on the principles of the Ghost-Fluid Method [15, 27]. This approach is widely used in the literature, even though it is only first-order accurate for solving the Poisson equation with jump conditions. 
In [21], a second-order approach developed, but it has not been applied to Navier-Stokes equations yet. The Ghost-Fluid Method introduced in [23] can be described briefly as proposed in [25]: first, the intermediate velocity $\mathbf{u}_{2}^{*}$ is updated with

$$
\mathbf{u}_{2}^{*}=\mathbf{u}^{n}-\Delta t\left(\left(\mathbf{u}^{n} \cdot \nabla\right) \mathbf{u}^{n}-\frac{\mu \Delta \mathbf{u}^{*}}{\rho^{n+1}}-\mathbf{g}\right) .
$$

Next, the Poisson equation for the pressure is solved with the appropriate jump condition:

$$
\nabla \cdot\left(\frac{\nabla p^{n+1}}{\rho^{n+1}}\right)=\frac{\nabla \cdot \mathbf{u}_{2}^{*}}{\Delta t}+\nabla \cdot\left(\frac{\sigma \kappa+2[\mu]\left(\mathbf{n}^{T} \cdot \nabla \mathbf{u} \cdot \mathbf{n}\right)^{n}}{\rho^{n+1}} \delta_{\Gamma} \mathbf{n}\right) .
$$

Finally the correction step gives the divergence free velocity at $t^{n+1}$ :

$$
\mathbf{u}^{n+1}=\mathbf{u}_{2}^{*}-\frac{\Delta t}{\rho^{n+1}}\left(\nabla p^{n+1}-\left(\sigma \kappa+2[\mu]\left(\mathbf{n}^{T} \cdot \nabla \mathbf{u} \cdot \mathbf{n}\right)^{n}\right) \mathbf{n} \delta_{\Gamma}\right) .
$$

This method has been dubbed the Ghost-Fluid Primitive viscous Method (GFPM) in [25]. In this framework, the computation of the viscous terms requires to determine explicitly the jump condition on the projected viscous stresses. As these jump conditions depend on the numerical solution, achieving a fully implicit temporal discretization of the viscous terms is not a straightforward task in the case of the GFPM. Depending on whether the CSF formulation or the GFPM formulation is used, the intermediate velocity in the prediction step is different. Even though these two methods are formally equivalent, they lead to different definitions of the predicted velocity field in the projection step.

\subsubsection{The Ghost-Fluid Conservative Viscous Method}

In [48], Sussman introduces another formulation for the intermediate velocity field, which [25] refers to as the Ghost-Fluid Conservative viscous Method (GFCM). In this case, the intermediate velocity $\mathbf{u}_{\mathbf{3}}^{*}$ is obtained as:

$$
\mathbf{u}_{\mathbf{3}}^{*}=\mathbf{u}^{n}-\Delta t\left(\left(\mathbf{u}^{\mathbf{n}} \cdot \nabla\right) \mathbf{u}^{n}-\frac{1}{\rho^{n+1}} \nabla \cdot\left(2 \mu \underline{\underline{\mathbf{D}}}^{n}\right)-\mathbf{g}\right) .
$$

Next, the Poisson equation is solved to determine the pressure field:

$$
\nabla \cdot\left(\frac{\nabla p^{n+1}}{\rho^{n+1}}\right)=\frac{\nabla \cdot \mathbf{u}_{3}^{*}}{\Delta t}+\nabla \cdot\left(\frac{\sigma \kappa \mathbf{n} \delta_{\Gamma}}{\rho^{n+1}}\right)
$$

Finally in the correction step, the physical velocity field can be determined with the pressure field previously computed:

$$
\mathbf{u}^{n+1}=\mathbf{u}_{3}^{*}-\frac{\Delta t}{\rho^{n+1}}\left(\nabla p^{n+1}-\sigma \kappa \mathbf{n} \delta_{\Gamma}\right) .
$$

In order to remove the $O\left(\Delta x^{2}\right)$ time step restriction incurred by the viscosity term, the following implicit temporal discretization is used, referred to in this paper as the Ghost-Fluid Conservative Method with an Implicit scheme (GFCMI): the intermediate velocity $\mathbf{u}_{\mathbf{4}}^{*}$ is updated as:

$$
\rho^{n+1} \mathbf{u}_{\mathbf{4}}^{*}-\Delta t \nabla \cdot\left(2 \mu{\underline{\underline{\mathbf{D}^{*}}}}^{*}=\rho^{n+1}\left(\mathbf{u}^{n}-\Delta t\left(\left(\mathbf{u}^{n} \cdot \nabla\right) \mathbf{u}^{n}-\mathbf{g}\right)\right),\right.
$$


which leads to a large linear system where the three velocity components are coupled. The subsequent steps are similar to the one used in GFCM, with the computation of the pressure field:

$$
\nabla \cdot\left(\frac{\nabla p^{n+1}}{\rho^{n+1}}\right)=\frac{\nabla \cdot \mathbf{u}_{4}^{*}}{\Delta t}+\nabla \cdot\left(\frac{\sigma \kappa \mathbf{n} \delta_{\Gamma}}{\rho^{n+1}}\right),
$$

followed by the correction step:

$$
\mathbf{u}^{n+1}=\mathbf{u}_{4}^{*}-\frac{\Delta t}{\rho^{n+1}}\left(\nabla p^{n+1}-\sigma \kappa \mathbf{n} \delta_{\Gamma}\right) .
$$

\subsubsection{The Ghost-Fluid Semi-Conservative Method}

Finally in [25] the authors proposed a semi-conservative formulation for discretizing the viscous term, referred to in this paper as the Ghost-Fluid Semi-Conservative Method with an Implicit scheme (GFSCMI). Starting with the splitting:

$$
\begin{aligned}
\nabla \cdot(2 \mu \underline{\underline{\mathbf{D}}}) & =\nabla \cdot\left(\mu \nabla \mathbf{u}+\mu \nabla^{T} \mathbf{u}\right) \\
& =\nabla \cdot(\mu \nabla \mathbf{u})+\nabla \cdot\left(\mu \nabla^{T} \mathbf{u}\right) \\
& =\nabla \cdot(\mu \nabla \mathbf{u})+\nabla^{T} \mathbf{u} \cdot \nabla \mu+\mu \nabla \cdot\left(\nabla^{T} \mathbf{u}\right) \\
& =\nabla \cdot(\mu \nabla \mathbf{u})+[\mu] \nabla^{T} \mathbf{u} \cdot \mathbf{n} \delta_{\Gamma},
\end{aligned}
$$

where $\nabla \cdot(\mu \nabla \mathbf{u})$ is a formal equivalent of the one-phase flow formulation of viscous effects. Therefore each component is solved independently from the others, as the cross-terms of the viscous term in $[\mu] \nabla^{T} \mathbf{u} \cdot \mathbf{n} \delta_{\Gamma}$ are enforced explicitly as a jump condition. The prediction step is:

$$
\rho^{n+1} \mathbf{u}_{5}^{*}-\Delta t \nabla \cdot\left(\mu \nabla \mathbf{u}_{5}^{*}\right)=\rho^{n+1}\left(\mathbf{u}^{n}-\Delta t\left(\left(\mathbf{u}^{n} \cdot \nabla\right) \mathbf{u}^{n}-\mathbf{g}\right)\right) .
$$

Then the computation of the pressure field takes into account part of the viscous effects in the pressure jump:

$$
\nabla \cdot\left(\frac{\nabla p^{n+1}}{\rho^{n+1}}\right)=\frac{\nabla \cdot \mathbf{u}_{5}^{*}}{\Delta t}+\nabla \cdot\left(\frac{\left(\sigma \kappa+[\mu]\left(\mathbf{n}^{T} \cdot \nabla \mathbf{u} \cdot \mathbf{n}\right)^{n}\right)}{\rho^{n+1}} \mathbf{n} \delta_{\Gamma}\right) .
$$

Finally, the correction step to compute the divergence free velocity is:

$$
\mathbf{u}^{n+1}=\mathbf{u}_{5}^{*}-\frac{\Delta t}{\rho^{n+1}}\left(\nabla p^{n+1}-\left(\sigma \kappa+[\mu]\left(\mathbf{n}^{T} \cdot \nabla \mathbf{u} \cdot \mathbf{n}\right)^{n}\right) \mathbf{n} \delta_{\Gamma}\right) .
$$

Remark: The implementation of an implicit temporal discretization of the viscous terms is easier with this method (GFSCMI) than GFCMI. Indeed if GFSCMI is used, each velocity component can be computed by solving a simple linear system (symmetric definite positive) that does not treat cross-derivatives implicitly. However, even though GFCMI treats cross-derivatives implicitly, a few iterations of Gauss-Seidel suffice for convergence and the results are superior to GFSCMI. These methods are compared in section 3.4 to offer some insight on how velocity boundary conditions should be enforced on irregular domains. 


\subsubsection{A Note on the Time Step Constraint}

Let us remark that, unlike GFCMI, GFSCMI does not fully remove the stability constraint on the time step due to viscosity, since an explicit part depending on the viscosity jump condition remains in equation (5). Therefore, as it has been discussed in [25], a stability constraint depending on the viscosity jump condition must be imposed to ensure stability of the method. Specifically, the following standard time step constraints for the convection and the surface tension effects, must be imposed to ensure the temporal stability of the computation:

$$
\Delta t_{\text {conv }}=\frac{\Delta x}{\max |\mathbf{u}|}, \quad \Delta t_{\text {surf_tens }}=\frac{1}{2} \sqrt{\frac{\rho_{l}}{\sigma}} \Delta x^{3 / 2},
$$

for a global time step restriction of:

$$
\frac{1}{\Delta t}=\frac{1}{\Delta t_{\text {conv }}}+\frac{1}{\Delta t_{\text {surf_tens }}}
$$

If GFSCMI is used, the following constraint must be added to account for the jump condition on viscosity in equation (5):

$$
\Delta t_{\mathrm{visc}}=\frac{\bar{\rho}}{[\mu]} \Delta x^{2},
$$

where $\bar{\rho}$ is the average density of the two phases. This leads to a new global time step restriction given by:

$$
\frac{1}{\Delta t}=\frac{1}{\Delta t_{\text {conv }}}+\frac{1}{\Delta t_{\text {surf_tens }}}+\frac{1}{\Delta t_{\text {visc }}} .
$$

\subsection{A Note on Solid's Boundary Conditions for Multiphase Flows}

As stated earlier, when irregular domains are handled, Neumann boundary conditions on the domain boundaries are enforced on $\Gamma_{s}$ the fluid-solid interface for the pressure field. It leads to the following relation for GFCMI:

$$
\left.\mathbf{n}_{s} \cdot \frac{\nabla p}{\rho}\right|_{\Gamma_{s}}=\left.\mathbf{n}_{s} \cdot\left(\mathbf{u}^{*}-\mathbf{u}_{s}+\sigma \kappa \mathbf{n} \delta_{\Gamma}\right)\right|_{\Gamma_{s}},
$$

and for GFSCMI as:

$$
\left.\mathbf{n}_{s} \cdot \frac{\nabla p}{\rho}\right|_{\Gamma_{s}}=\left.\mathbf{n}_{s} \cdot\left(\mathbf{u}^{*}-\mathbf{u}_{s}+\left(\sigma \kappa+[\mu]\left(\mathbf{n}^{T} \cdot \nabla \mathbf{u} \cdot \mathbf{n}\right)^{n}\right) \mathbf{n} \delta_{\Gamma}\right)\right|_{\Gamma_{s}} .
$$

One can notice that jump conditions have to be taken into account on the irregular domain as well. Section 3 will discuss the numerical discretization of these boundary conditions.

\section{Numerical Methods}

In this section, we provide the details of the numerical methods used in our two-phase flow solver in irregular domains. First, we present the level-set method and the spatial discretizations of the two different projection methods that we consider for irregular domains. We then provide the details of the implicit discretization of the viscous terms how the level-set function is computed in the solid domain in order to impose the contact angle. 


\subsection{Capturing the Moving Interface}

In this work, we use the level-set method of Osher and Sethian [36] to represent the interface between the two fluids. A signed and continuous level-set function $\phi$ is defined in a domain $\Omega$ such as $\Omega^{-}=\{\mathbf{x}: \phi(\mathbf{x})<0\}, \Omega^{+}\{\mathbf{x}: \phi(\mathbf{x})>0\}$ and $\Gamma=\{\mathbf{x}: \phi(\mathbf{x})=0\}$ where $\Gamma$ represents the interface captured by the following transport equation:

$$
\frac{\partial \phi}{\partial t}+\mathbf{u} \cdot \nabla \phi=0
$$

One of the benefits for using the level-set function is its regular property in the whole domain. Sussman et al. [47] developed an algorithm allowing to maintain this property by re-initializing the level-set function with the following equation:

$$
\frac{\partial d}{\partial \tau}=\operatorname{Sign}(\phi)(1-|\nabla d|),
$$

where $\operatorname{Sign}(\phi)$ is a regularized signed function defined by Sussman et al. in [47] and $d$ the update of the function $\phi$. Equation (8) iterates for a few fictitious time step $\tau$ to finally converge to a continuous signed distance function in the whole domain. Thus, geometric properties such as $\mathbf{n}$ the outward unit normal vector to the interface and $\kappa$ the local mean curvature can be accurately computed:

$$
\mathbf{n}=\frac{\nabla \phi}{|\nabla \phi|}, \quad \text { and } \quad \kappa(\phi)=\nabla \cdot \mathbf{n} .
$$

Spatial derivatives in equations (7) and (8) are computed with the WENO-Z scheme [5], and the temporal derivative with a third order TVD Runge-Kutta scheme. Let us notice that the temporal integration is fully coupled with the Navier-Stokes solver [23].

\subsection{Discretization of the Projection Method in Irregular Domains}

The method developed in this study to solve the Poisson equation (4) on irregular domains is based on Ng, Min and Gibou's algorithm [34]. This numerical method presents several attractive features. Indeed the spatial discretization for the pressure Poisson equation is second order, for all computational nodes, including the irregular domain nodes crossed by the solid-fluid interface where the embedded Neumann Boundary condition is enforced. Moreover, the resulting matrix of the linear system is still symmetric definite positive which allows using a classical Preconditionned Conjugate Gradient or a Black Box MultiGrid solver [13].

Consider a vector field $\mathbf{u}^{*}$ in the domain $\Omega$, separated by the interface $\Gamma_{s}$ into two distinct subdomains $\Omega_{f}$ and $\Omega_{s}$, such as $\Omega=\Omega_{f} \cup \Omega_{s}$. As $\Omega_{s}$ would correspond to a solid media, we only solve the Navier-Stokes equations in $\Omega_{f}$. Let $\phi_{s}$ be another level-set function such as $\Omega_{s}=\{\mathbf{x}$ : $\left.\phi_{s}(\mathbf{x})>0\right\}, \Omega_{f}\left\{\mathbf{x}: \phi_{s}(\mathbf{x})<0\right\}$ and $\Gamma_{s}=\left\{\mathbf{x}: \phi_{s}(\mathbf{x})=0\right\}$. We consider a MAC grid configuration and a cell $C_{i, j}=[i-1 / 2, i+1 / 2] \mathrm{x}[j-1 / 2, j+1 / 2]$, partially covered with $\Omega_{f}$. For more clarity we only consider a $2 \mathrm{D}$ example to illustrate the method which can be extended to $3 \mathrm{D}$. The configuration is illustrated in Fig 2.

Considering a finite volume approach, we integrate the left hand side of equation (4) over $C_{i, j}$ and apply the divergence theorem:

$$
\int_{C_{i, j} \cap \Omega_{f}} \nabla \cdot\left(\frac{\nabla p}{\rho}\right) d A=\int_{\partial\left(C_{i, j} \cap \Omega_{f}\right)} \mathbf{n} \cdot\left(\frac{\nabla p}{\rho}\right) d l,
$$




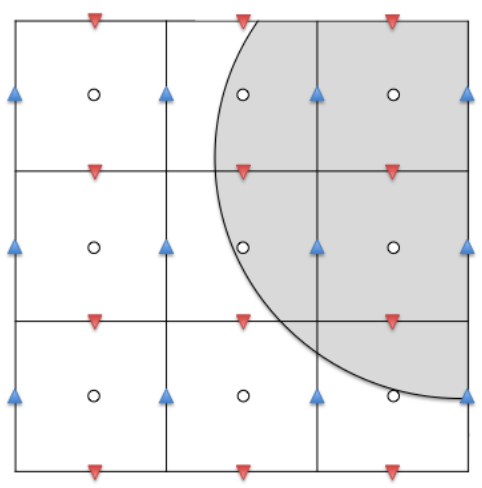

Figure 2: Standard MAC grid configuration: the scalar variables are sampled at the cells' centers (circles), the xcomponent of the velocity field is sampled on the vertical faces (blue triangles), and the y-component of the velocity field is sampled on the horizontal faces (red triangles). The irregular domain is represented by the shaded area $\Omega_{s}$.

with $d A$ and $d l$ differential area and length. Similarly for the right hand side:

$$
\int_{C_{i, j} \cap \Omega_{f}} \nabla \cdot \mathbf{u}^{*} d A+\int_{C_{i, j} \cap \Omega_{f}} \nabla \cdot\left(\frac{\mathbf{F}_{S T}}{\rho}\right) d A=\int_{\partial\left(C_{i, j} \cap \Omega_{f}\right)} \mathbf{n} \cdot \mathbf{u}^{*} d l+\int_{\partial\left(C_{i, j} \cap \Omega_{f}\right)} \mathbf{n} \cdot\left(\frac{\mathbf{F}_{S T}}{\rho}\right) d l,
$$

where $\mathbf{F}_{S T}$ can represent the surface tension effects and the jump conditions due to viscosity depending on the choice of the intermediate velocity:

- For GFCMI, $\mathbf{F}_{S T}=\sigma \kappa \mathbf{n}_{\mathbf{f}} \delta_{f}$.

- For GFSCMI, $\mathbf{F}_{S T}=\left(\sigma \kappa+[\mu]_{\Gamma}\left(\mathbf{n}^{T} \cdot \nabla \mathbf{u} \cdot \mathbf{n}\right)^{n}\right) \mathbf{n} \delta_{f}$.

We now only consider the contribution of all the components of $\partial\left(C_{i, j} \cap \Omega_{f}\right)$, and define the length fraction $L_{i, j}$ of the face covered by the irregular domain $\left\{x \mid \phi_{s}(x) \leq 0\right\}$ which can be linearly approximated here for $\left[i-\frac{1}{2}\right] \times\left[j-\frac{1}{2}, j+\frac{1}{2}\right]$ by:

$$
L_{i-\frac{1}{2}, j}=\left\{\begin{array}{lll}
\Delta y \frac{\phi_{i-\frac{1}{2}, j-\frac{1}{2}}}{\phi_{i-\frac{1}{2}, j-\frac{1}{2}}-\phi_{i-\frac{1}{2}, j+\frac{1}{2}}} & \text { for } \phi_{i-\frac{1}{2}, j-\frac{1}{2}}<0 & \text { and } \phi_{i-\frac{1}{2}, j \frac{1}{2}}>0, \\
\Delta y \frac{\phi_{i-\frac{1}{2}, j+\frac{1}{2}}}{\phi_{i-\frac{1}{2}, j+\frac{1}{2}}-\phi_{i-\frac{1}{2}, j-\frac{1}{2}}} & \text { for } \phi_{i-\frac{1}{2}, j-\frac{1}{2}}>0 & \text { and } \phi_{i-\frac{1}{2}, j \frac{1}{2}<0} \\
\Delta y & \text { for } \phi_{i-\frac{1}{2}, j-\frac{1}{2}}<0 & \text { and } \phi_{i-\frac{1}{2}, j \frac{1}{2}<0}< \\
0 & \text { for } \phi_{i-\frac{1}{2}, j-\frac{1}{2}}>0 & \text { and } \phi_{i-\frac{1}{2}, j \frac{1}{2}}>0 .
\end{array}\right.
$$

For more clarity the subscript " $s$ " is omitted, but it is the function $\phi_{s}$ that is used to estimate the length fractions. Using the length fraction established in equation (9), and sampled values at the center of the cell, we obtain: 


$$
\begin{aligned}
-\int_{\partial\left(C_{i, j} \cap \Omega_{f}\right)} \mathbf{n} \cdot\left(\frac{\nabla p}{\rho}\right) d l & \simeq \frac{L_{i-\frac{1}{2}, j}}{\rho_{i-\frac{1}{2}, j}}\left(\frac{p_{i, j}-p_{i-1, j}}{\Delta x}\right)+\frac{L_{i+\frac{1}{2}, j}}{\rho_{i+\frac{1}{2}, j}}\left(\frac{p_{i, j}-p_{i+1, j}}{\Delta x}\right) \\
& +\frac{L_{i, j-\frac{1}{2}}}{\rho_{i, j-\frac{1}{2}}}\left(\frac{p_{i, j}-p_{i, j-1}}{\Delta y}\right)+\frac{L_{i, j+\frac{1}{2}}}{\rho_{i, j+\frac{1}{2}}}\left(\frac{p_{i, j}-p_{i, j+1}}{\Delta y}\right) \\
& -\int_{C_{i, j} \cap \Gamma} \mathbf{n} \cdot\left(\frac{\nabla p}{\rho}\right) d l,
\end{aligned}
$$

where $\int_{C_{i, j} \cap \Gamma}$ is the integral over the interface with the irregular external boundary. Similarly, we obtain:

$$
\begin{aligned}
-\int_{\partial\left(C_{i, j} \cap \Omega_{f}\right)} \mathbf{n} \cdot \mathbf{u}^{*} d l & \simeq L_{i-\frac{1}{2}, j} u_{i-\frac{1}{2}, j}^{*}-L_{i+\frac{1}{2}, j} u_{i+\frac{1}{2}, j}^{*}+L_{i, j-\frac{1}{2}} v_{i, j-\frac{1}{2}}^{*}-L_{i, j+\frac{1}{2}} v_{i, j+\frac{1}{2}}^{*} \\
& -\int_{C_{i, j} \cap \Gamma} \mathbf{n} \cdot \mathbf{u}^{*} d l .
\end{aligned}
$$

For cells where both $\Gamma$ and $\Gamma_{s}$ are present, respectively the fluid-fluid interface and the fluid-solid interface, the Ghost-Fluid method [15, 27] is applied on each part of $\Gamma$ : let $\beta_{i, j}=\frac{1}{\rho_{i, j}}$ be a diffusion coefficient in the cell, computed with a harmonic average of the values $\beta^{+}$in the region where $\phi$ is positive and $\beta^{-}$for the negative region, and $a\left(x_{\Gamma}\right)=\sigma \kappa$ the corresponding jump function for GFCMI. For the interface crossing a cell border between $x_{i, j}$ and $x_{i+1, j}$ :

$$
\beta_{i+1 / 2, j}=\frac{\beta^{+} \beta^{-}}{\beta^{+} \theta+\beta^{-}(1-\theta)},
$$

and

$$
a\left(x_{\Gamma}\right)_{i+1 / 2, j}=\sigma \kappa_{i, j} \theta+\sigma \kappa_{i+1, j}(1-\theta)
$$

with

$$
\theta=\frac{\left|\phi_{i+1, j}\right|}{\left|\phi_{i, j}\right|+\left|\phi_{i+1, j}\right|} .
$$

Then one can define $g_{i, j}^{*}$ as in [25]:

$g_{i, j}^{L}= \pm \beta_{i-\frac{1}{2}, j} a\left(x_{\Gamma}\right)_{i-\frac{1}{2}, j} \quad g_{i, j}^{R}= \pm \beta_{i+\frac{1}{2}, j} a\left(x_{\Gamma}\right)_{i+\frac{1}{2}, j} \quad g_{i, j}^{B}= \pm \beta_{i, j-\frac{1}{2}} a\left(x_{\Gamma}\right)_{i, j-\frac{1}{2}} \quad g_{i, j}^{T}= \pm \beta_{i, j+\frac{1}{2}} a\left(x_{\Gamma}\right)_{i, j+\frac{1}{2}}$

and finally obtain :

$$
\begin{aligned}
-\int_{\partial\left(C_{i, j} \cap \Omega^{-}\right)} \mathbf{n} \cdot\left(\frac{\sigma \kappa \mathbf{n}_{\mathbf{f}} \delta_{f}}{\rho}\right) d l & \simeq L_{i-\frac{1}{2}, j} g_{i, j}^{L}+L_{i+\frac{1}{2}, j} g_{i, j}^{R}+L_{i, j-\frac{1}{2}} g_{i, j}^{B}+L_{i, j+\frac{1}{2}} g_{i, j}^{T} \\
& -\int_{C_{i, j} \cap \Gamma} \mathbf{n} \cdot\left(\frac{\sigma \kappa \mathbf{n}_{\mathbf{f}} \delta_{f}}{\rho}\right) d l .
\end{aligned}
$$

If GFSCMI is used, one can notice that the formulation is almost the same, except that the function $a\left(x_{\Gamma}\right)$ will be completed with the jump due to the viscous term:

$$
a\left(x_{\Gamma}\right)_{i+1 / 2, j}=\theta\left(\sigma \kappa+[\mu]_{\Gamma}\left(\mathbf{n}^{T} \cdot \nabla \mathbf{u} \cdot \mathbf{n}\right)^{n}\right)_{i, j}+(1-\theta)\left(\sigma \kappa+[\mu]_{\Gamma}\left(\mathbf{n}^{T} \cdot \nabla \mathbf{u} \cdot \mathbf{n}\right)^{n}\right)_{i+1, j},
$$


and

$$
\begin{aligned}
-\int_{\partial\left(C_{i, j} \cap \Omega^{-}\right)} \mathbf{n} \cdot\left(\frac{\left(\sigma \kappa+[\mu]_{\Gamma}\left(\mathbf{n}^{T} \cdot \nabla \mathbf{u} \cdot \mathbf{n}\right)^{n}\right) \mathbf{n}_{\mathbf{f}} \delta_{f}}{\rho}\right) d l & \simeq L_{i-\frac{1}{2}, j} g_{i, j}^{L}+L_{i+\frac{1}{2}, j} g_{i, j}^{R}+L_{i, j-\frac{1}{2}} g_{i, j}^{B}+L_{i, j+\frac{1}{2}} g_{i, j}^{T} \\
& -\int_{C_{i, j} \cap \Gamma} \mathbf{n} \cdot\left(\frac{\left(\sigma \kappa+[\mu]_{\Gamma}\left(\mathbf{n}^{T} \cdot \nabla \mathbf{u} \cdot \mathbf{n}\right)^{n}\right) \mathbf{n}_{\mathbf{f}} \delta_{f}}{\rho}\right) d l .
\end{aligned}
$$

Finally, one obtains the following problem with Neumann boundary conditions on the embedded solid boundary:

$$
\begin{aligned}
& \frac{L_{i-\frac{1}{2}, j}}{\rho_{i-\frac{1}{2}, j}}\left(\frac{p_{i, j}-p_{i-1, j}}{\Delta x}\right)+\frac{L_{i+\frac{1}{2}, j}}{\rho_{i+\frac{1}{2}, j}}\left(\frac{p_{i, j}-p_{i+1, j}}{\Delta x}\right)+\frac{L_{i, j-\frac{1}{2}}}{\rho_{i, j-\frac{1}{2}}}\left(\frac{p_{i, j}-p_{i, j-1}}{\Delta y}\right)+\frac{L_{i, j+\frac{1}{2}}}{\rho_{i, j+\frac{1}{2}}}\left(\frac{p_{i, j}-p_{i, j+1}}{\Delta y}\right) \\
& =L_{i-\frac{1}{2}, j} u_{i-\frac{1}{2}, j}^{*}-L_{i+\frac{1}{2}, j} u_{i+\frac{1}{2}, j}^{*}+L_{i, j-\frac{1}{2}} v_{i, j-\frac{1}{2}}^{*}-L_{i, j+\frac{1}{2}} v_{i, j+\frac{1}{2}}^{*} \\
& +L_{i-\frac{1}{2}, j} g_{i, j}^{L}+L_{i+\frac{1}{2}, j} g_{i, j}^{R}+L_{i, j-\frac{1}{2}} g_{i, j}^{B}+L_{i, j+\frac{1}{2}} g_{i, j}^{T} .
\end{aligned}
$$

As Ng, Min and Gibou demonstrated in [34], the above discretization forms a symmetric positive definite linear system for the pressure field.

\subsection{Spatial discretization of the viscous terms}

As illustrated in Section 2, the implementation of the viscosity terms depends on the formulation of the intermediate velocity, thus generating different discretizations that are formally equivalents. The implicit formulations for both GFCM and GFSCM are thoroughly detailed in the following section.

\subsubsection{Viscosity Modeling - GFCMI Equation}

We have seen in section 2 that the equation of the intermediate velocity for GFCMI is:

$$
\rho^{n+1} \mathbf{U}^{*}-\Delta t\left(\nabla \cdot\left(2 \mu \underline{\underline{\mathbf{D}}}^{*}\right)\right)=\mathbf{F}_{\text {rhs }},
$$

with $\mathbf{F}_{\text {rhs }}$ the right-hand side of equation (10):

$$
\mathbf{F}_{\mathbf{r h s}}=\rho^{n+1}\left(\mathbf{u}^{n}-\Delta t\left(\left(\mathbf{U}^{\mathbf{n}} \cdot \nabla\right) \mathbf{U}^{\mathbf{n}}-\mathbf{g}\right)\right) .
$$

In order to compute the intermediate velocity field, one must compute all the terms of the right-hand side of equation (11). The advection term is solved with a fifth order WENO-Z scheme [5]. Let us remind the stress tensor formulation for the discretization of the viscous term:

$$
\underline{\underline{\mathbf{D}}}=\frac{1}{2}\left(\nabla \mathbf{u}+\nabla^{T} \mathbf{u}\right) \quad \text { with } \quad \nabla \mathbf{u}=\left(\begin{array}{ll}
\frac{\partial u}{\partial x} & \frac{\partial u}{\partial y} \\
\frac{\partial v}{\partial x} & \frac{\partial v}{\partial y}
\end{array}\right) .
$$


The discretization is presented as a $2 \mathrm{D}$ discretization, but can be easily extended to a 3D formulation. By applying the divergence operator:

$$
\nabla \cdot(2 \mu \underline{\underline{\mathbf{D}}})=\left(\begin{array}{l}
\frac{\partial}{\partial x}\left(2 \mu \frac{\partial u}{\partial x}\right)+\frac{\partial}{\partial y}\left(\mu\left(\frac{\partial u}{\partial y}+\frac{\partial v}{\partial x}\right)\right) \\
\frac{\partial}{\partial y}\left(2 \mu \frac{\partial v}{\partial y}\right)+\frac{\partial}{\partial x}\left(\mu\left(\frac{\partial u}{\partial y}+\frac{\partial v}{\partial x}\right)\right)
\end{array}\right) .
$$

By projecting on the $\mathbf{e}_{x}$ direction:

$$
\left.\nabla \cdot(2 \mu \underline{\underline{\mathbf{D}}}) \cdot \mathbf{e}_{x}\right|_{i+\frac{1}{2}, j}=\left.\frac{\partial}{\partial x}\left(2 \mu \frac{\partial u}{\partial x}\right)\right|_{i+\frac{1}{2}, j}+\left.\frac{\partial}{\partial y}\left(\mu\left(\frac{\partial u}{\partial y}+\frac{\partial v}{\partial x}\right)\right)\right|_{i+\frac{1}{2}, j},
$$

with:

$$
\begin{gathered}
\left.\frac{\partial}{\partial x}\left(2 \mu \frac{\partial u}{\partial x}\right)\right|_{i+\frac{1}{2}, j} \simeq \frac{2 \mu_{i+1, j}\left(\frac{u_{i+\frac{3}{2} j}-u_{i+\frac{1}{2}, j}}{\Delta x}\right)-2 \mu_{i, j}\left(\frac{u_{i+\frac{1}{2}, j}-u_{i-\frac{1}{2}, j}}{\Delta x}\right)}{\Delta x}, \\
\left.\frac{\partial}{\partial y}\left(\mu \frac{\partial u}{\partial y}\right)\right|_{i+\frac{1}{2}, j} \simeq \frac{\mu_{i+\frac{1}{2}, j+\frac{1}{2}}\left(\frac{u_{i+\frac{1}{2}, j+1}-u_{i+\frac{1}{2}, j}}{\Delta y}\right)-\mu_{i+\frac{1}{2}, j-\frac{1}{2}}\left(\frac{u_{i+\frac{1}{2}, j}-u_{i+\frac{1}{2}, j-1}}{\Delta y}\right)}{\Delta y},
\end{gathered}
$$

and

$$
\left.\frac{\partial}{\partial y}\left(\mu \frac{\partial v}{\partial x}\right)\right|_{i+\frac{1}{2}, j} \simeq \frac{\mu_{i+\frac{1}{2}, j+\frac{1}{2}}\left(\frac{v_{i+1, j+\frac{1}{2}}-v_{i, j+\frac{1}{2}}}{\Delta x}\right)-\mu_{i+\frac{1}{2}, j-\frac{1}{2}}\left(\frac{v_{i+1, j-\frac{1}{2}}-v_{i, j-\frac{1}{2}}}{\Delta x}\right)}{\Delta y} .
$$

Likewise, by projecting on the $\left.\mathbf{e}_{y}\right|_{i, j+\frac{1}{2}}$ direction:

$$
\left.\nabla \cdot(2 \mu \underline{\underline{\mathbf{D}}}) \cdot \mathbf{e}_{y}\right|_{i, j+\frac{1}{2}}=\left.\frac{\partial}{\partial x}\left(\mu\left(\frac{\partial u}{\partial y}+\frac{\partial v}{\partial x}\right)\right)\right|_{i, j+\frac{1}{2}}+\left.\frac{\partial}{\partial y}\left(2 \mu \frac{\partial v}{\partial y}\right)\right|_{i, j+\frac{1}{2}},
$$

with:

$$
\begin{gathered}
\left.\frac{\partial}{\partial x}\left(\mu \frac{\partial u}{\partial y}\right)\right|_{i, j+\frac{1}{2}} \simeq \frac{\mu_{i+\frac{1}{2}, j+\frac{1}{2}}\left(\frac{u_{i+\frac{1}{2}, j+1}-u_{i+\frac{1}{2}, j}}{\Delta y}\right)-\mu_{i-\frac{1}{2}, j+\frac{1}{2}}\left(\frac{u_{i-\frac{1}{2}, j+1}-u_{i-\frac{1}{2}, j}}{\Delta y}\right)}{\Delta x}, \\
\left.\frac{\partial}{\partial x}\left(\mu \frac{\partial v}{\partial x}\right)\right|_{i, j+\frac{1}{2}} \simeq \frac{\mu_{i+\frac{1}{2}, j+\frac{1}{2}}\left(\frac{v_{i+1, j+\frac{1}{2}}-v_{i, j+\frac{1}{2}}}{\Delta x}\right)-\mu_{i-\frac{1}{2}, j+\frac{1}{2}}\left(\frac{v_{i, j+\frac{1}{2}}-v_{i-1 j+\frac{1}{2}}}{\Delta x}\right)}{\Delta x},
\end{gathered}
$$

and

$$
\left.\frac{\partial}{\partial y}\left(2 \mu \frac{\partial v}{\partial y}\right)\right|_{i, j+\frac{1}{2}} \simeq \frac{2 \mu_{i, j+1}\left(\frac{v_{i, j+\frac{3}{2}}-v_{i, j+\frac{1}{2}}}{\Delta y}\right)-2 \mu_{i, j}\left(\frac{v_{i, j+\frac{1}{2}}-v_{i, j-\frac{1}{2}}}{\Delta y}\right)}{\Delta y} .
$$


In order to compute rightfully the viscous term at the intermediate time step, one must solve a coupled linear system of two matrices with 9 diagonals per velocity components as:

$$
\begin{array}{r}
a_{i+1, j} u_{i+\frac{3}{2}, j}^{*}+a_{i, j} u_{i-\frac{1}{2}, j}^{*}+a_{i+\frac{1}{2}, j+\frac{1}{2}} u_{i+\frac{1}{2}, j+1}^{*}+a_{i+\frac{1}{2}, j-\frac{1}{2}} u_{i+\frac{1}{2}, j-1}^{*}+\alpha u_{i+\frac{1}{2}, j}^{*} \\
-b_{i+\frac{1}{2}, j+\frac{1}{2}} v_{i+1, j+\frac{1}{2}}^{*}+b_{i+\frac{1}{2}, j+\frac{1}{2}} v_{i, j+\frac{1}{2}}^{*}-b_{i+\frac{1}{2}, j-\frac{1}{2}} v_{i+1, j-\frac{1}{2}}^{*}+b_{i-\frac{1}{2}, j-\frac{1}{2}} v_{i, j-\frac{1}{2}}^{*}=F_{\text {X_rhs }}
\end{array}
$$

and

$$
\begin{array}{r}
c_{i+\frac{1}{2}, j+\frac{1}{2}} v_{i+1, j+\frac{1}{2}}^{*}+c_{i-\frac{1}{2}, j+\frac{1}{2}} v_{i-1 j+\frac{1}{2}}^{*}+c_{i, j+1} v_{i, j+\frac{3}{2}}^{*}+c_{i, j} v_{i, j-\frac{1}{2}}^{*}+\beta v_{i, j+\frac{1}{2}}^{*} \\
-b_{i+\frac{1}{2}, j+\frac{1}{2}} u_{i+\frac{1}{2}, j+1}^{*}-b_{i-\frac{1}{2}, j+\frac{1}{2}} u_{i+\frac{1}{2}, j}^{*}+b_{i+\frac{1}{2}, j+\frac{1}{2}} u_{i-\frac{1}{2}, j+1}^{*}+b_{i-\frac{1}{2}, j+\frac{1}{2}} u_{i-\frac{1}{2}, j}^{*}=F_{\mathrm{y} \_ \text {rhs }}
\end{array}
$$

with $F_{\mathrm{x} \_ \text {rhs }}$ and $F_{\mathrm{y}_{-} \text {rhs }}$ the right-hand side terms from GFCMI projection method in equation (11), respectively projected onto the $x$ - and $y$ - directions, respectively. The matrix coefficients are deduced from equations (10), (12) and (13) as:

$$
\begin{aligned}
& a_{i+1, j}=-\frac{2 \mu_{i+1, j}}{\Delta x^{2}} \Delta t, \quad b_{i+\frac{1}{2}, j+\frac{1}{2}}=\frac{\mu_{i+\frac{1}{2}, j+\frac{1}{2}}}{\Delta x \Delta y} \Delta t, \quad c_{i+\frac{1}{2}, j+\frac{1}{2}}=-\frac{\mu_{i+\frac{1}{2}, j+\frac{1}{2}}}{\Delta x^{2}} \Delta t, \\
& a_{i, j}=-\frac{2 \mu_{i, j}}{\Delta x^{2}} \Delta t, \quad b_{i+\frac{1}{2}, j-\frac{1}{2}}=\frac{\mu_{i+\frac{1}{2}, j-\frac{1}{2}}}{\Delta x \Delta y} \Delta t, \quad c_{i-\frac{1}{2}, j+\frac{1}{2}}=-\frac{\mu_{i-\frac{1}{2}, j+\frac{1}{2}}}{\Delta x^{2}} \Delta t, \\
& a_{i+\frac{1}{2}, j+\frac{1}{2}}=-\frac{\mu_{i+\frac{1}{2}, j+\frac{1}{2}}}{\Delta y^{2}} \Delta t, \quad b_{i-\frac{1}{2}, j+\frac{1}{2}}=\frac{\mu_{i-\frac{1}{2}, j+\frac{1}{2}}}{\Delta x \Delta y} \Delta t, \quad c_{i, j+1}=-\frac{2 \mu_{i, j+1}}{\Delta y^{2}} \Delta t, \\
& a_{i+\frac{1}{2}, j-\frac{1}{2}}=-\frac{\mu_{i+\frac{1}{2}, j-\frac{1}{2}}}{\Delta y^{2}} \Delta t, \quad b_{i-\frac{1}{2}, j-\frac{1}{2}}=\frac{\mu_{i-\frac{1}{2}, j-\frac{1}{2}}}{\Delta x \Delta y} \Delta t, \quad c_{i, j}=-\frac{2 \mu_{i, j}}{\Delta y^{2}} \Delta t, \\
& \alpha=\rho_{i+\frac{1}{2}, j}^{n+1}-a_{i+1, j}-a_{i, j}-a_{i+\frac{1}{2}, j+\frac{1}{2}}-a_{i+\frac{1}{2}, j-\frac{1}{2}},
\end{aligned}
$$

and

$$
\beta=\rho_{i, j+\frac{1}{2}}^{n+1}-c_{i+\frac{1}{2}, j+\frac{1}{2}}-c_{i-\frac{1}{2}, j+\frac{1}{2}}-c_{i, j+1}-c_{i, j} .
$$

Since the linear system is diagonally dominant ${ }^{2}$, it can be solved efficiently with a few GaussSeidel iterations $(<20$ iterations for typical multiphase flow configurations, such as the benchmarks of section 4). For a 3D system, a third coupled matrix has to be accounted for, resulting in a 15diagonal matrix per velocity components.

\subsubsection{Viscosity Modeling - GFSCMI Equation}

GFSCMI projection proposed in [25] enables an easier implementation for an implicit temporal discretization of the viscous terms, albeit semi-implicit:

$$
\rho^{n+1} \mathbf{u}_{5}^{*}-\Delta t \nabla \cdot\left(\mu \nabla \mathbf{u}_{5}^{*}\right)=\mathbf{F}_{\mathbf{r h s}}=\rho^{n+1}\left(\mathbf{u}^{n}-\Delta t\left(\left(\mathbf{u}^{n} \cdot \nabla\right) \mathbf{u}^{n}-\mathbf{g}\right)\right) .
$$

\footnotetext{
${ }^{2}$ Depending on the time step constraint, if $\rho>>\Sigma a_{i j}$, then the system is diagonally dominant, which is always the case in our configurations, cf. Appendix B
} 
The viscous term can be computed as:

$$
\nabla \cdot(\mu \nabla \mathbf{u})=\left(\begin{array}{c}
\frac{\partial}{\partial x}\left(\mu \frac{\partial u}{\partial x}\right)+\frac{\partial}{\partial y}\left(\mu \frac{\partial u}{\partial y}\right) \\
\frac{\partial}{\partial x}\left(\mu \frac{\partial v}{\partial x}\right)+\frac{\partial}{\partial y}\left(\mu \frac{\partial v}{\partial y}\right)
\end{array}\right) .
$$

By projecting in the $x$-direction:

$$
\left.\nabla \cdot(\mu \nabla \mathbf{u}) \cdot \mathbf{e}_{x}\right|_{i+\frac{1}{2}, j}=\left.\frac{\partial}{\partial x}\left(\mu \frac{\partial u}{\partial x}\right)\right|_{i+\frac{1}{2}, j}+\left.\frac{\partial}{\partial y}\left(\mu \frac{\partial u}{\partial y}\right)\right|_{i+\frac{1}{2}, j},
$$

with:

$$
\left.\frac{\partial}{\partial x}\left(\mu \frac{\partial u}{\partial x}\right)\right|_{i+\frac{1}{2}, j} \simeq \frac{\mu_{i+1, j}\left(\frac{u_{i+\frac{3}{2} j}-u_{i+\frac{1}{2}, j}}{\Delta x}\right)-\mu_{i, j}\left(\frac{u_{i+\frac{1}{2}, j}-u_{i-\frac{1}{2}, j}}{\Delta x}\right)}{\Delta x},
$$

and

$$
\left.\frac{\partial}{\partial y}\left(\mu \frac{\partial u}{\partial y}\right)\right|_{i+\frac{1}{2}, j} \simeq \frac{\mu_{i+\frac{1}{2}, j+\frac{1}{2}}\left(\frac{u_{i+\frac{1}{2}, j+1}-u_{i+\frac{1}{2}, j}}{\Delta y}\right)-\mu_{i+\frac{1}{2}, j-\frac{1}{2}}\left(\frac{u_{i+\frac{1}{2}, j}-u_{i+\frac{1}{2}, j-1}}{\Delta y}\right)}{\Delta y} .
$$

Likewise, by projecting in the $y$-direction:

$$
\left.\nabla \cdot(\mu \nabla \mathbf{u}) \cdot \mathbf{e}_{y}\right|_{i, j+\frac{1}{2}}=\left.\frac{\partial}{\partial x}\left(\mu \frac{\partial v}{\partial x}\right)\right|_{i, j+\frac{1}{2}}+\left.\frac{\partial}{\partial y}\left(\mu \frac{\partial v}{\partial y}\right)\right|_{i, j+\frac{1}{2}},
$$

with:

$$
\left.\frac{\partial}{\partial x}\left(\mu \frac{\partial v}{\partial x}\right)\right|_{i, j+\frac{1}{2}} \simeq \frac{\mu_{i+\frac{1}{2}, j+\frac{1}{2}}\left(\frac{v_{i+1, j+\frac{1}{2}}-v_{i, j+\frac{1}{2}}}{\Delta x}\right)-\mu_{i-\frac{1}{2}, j+\frac{1}{2}}\left(\frac{v_{i, j+\frac{1}{2}}-v_{i-1 j+\frac{1}{2}}}{\Delta x}\right)}{\Delta x},
$$

and

$$
\left.\frac{\partial}{\partial y}\left(\mu \frac{\partial v}{\partial y}\right)\right|_{i, j+\frac{1}{2}} \simeq \frac{\mu_{i, j+1}\left(\frac{v_{i, j+\frac{3}{2}}-v_{i, j+\frac{1}{2}}}{\Delta y}\right)-\mu_{i, j}\left(\frac{v_{i, j+\frac{1}{2}}-v_{i, j-\frac{1}{2}}}{\Delta y}\right)}{\Delta y} .
$$

In order to solve for the viscous term in the intermediate time step, one must solve a linear system in each direction:

$$
a_{i+1, j} u_{i+\frac{3}{2} j}^{*}+a_{i, j} u_{i-\frac{1}{2}, j}^{*}+a_{i+\frac{1}{2}, j+\frac{1}{2}} u_{i+\frac{1}{2}, j+1}^{*}+a_{i+\frac{1}{2}, j-\frac{1}{2}} u_{i+\frac{1}{2}, j-1}^{*}+\alpha u_{i+\frac{1}{2}, j}^{*}=F_{\mathrm{x} \_\mathrm{rhs}},
$$

and

$$
c_{i+\frac{1}{2}, j+\frac{1}{2}} v_{i+1, j+\frac{1}{2}}^{*}+c_{i-\frac{1}{2}, j+\frac{1}{2}} v_{i-1 j+\frac{1}{2}}^{*}+c_{i, j+1} v_{i, j+\frac{3}{2}}^{*}+c_{i, j} v_{i, j-\frac{1}{2}}^{*}+\beta v_{i, j+\frac{1}{2}}^{*}=F_{\mathrm{y} \_\mathrm{rhs}},
$$


with $F_{\mathrm{x}_{\text {rns }}}$ and $F_{\mathrm{y} \_ \text {rhs }}$ the right-hand side terms from GFSCMI projection method in Eq. (17), respectively projected onto the $x$ - and $y$ - direction. The coefficients above are given by equations (14), (15) and (16).

As explained before, this formulation is quite straightforward as each component is decoupled from the others. This section is concluded by some comments on how to define the density $\rho_{i, j+\frac{1}{2}}^{n+1}$

and $\rho_{i+\frac{1}{2}, j}^{n+1}$, as well as all the viscosity constants $\mu_{i \pm \frac{1}{2} j \pm \frac{1}{2}}$. The definitions must be compatible with the computation of the correction step, which itself relies on the way the density is computed when the Poisson equation for the pressure is solved. We use the accurate computation of the harmonic average when grid cells are crossed by the fluid-fluid interface, as proposed in [23, 27]:

$$
\frac{1}{\rho^{n+1}}=\frac{\frac{1}{\rho^{+} \rho^{-}}}{\frac{1}{\rho^{+}} \theta+\frac{1}{\rho^{-}}(1-\theta)}=\frac{1}{\rho^{-} \theta+\rho^{+}(1-\theta)} .
$$

\subsection{Velocity Boundary Conditions}

On the solid-fluid interface, we impose the following Dirichlet boundary condition to enforce the solid's velocity $\mathbf{u}_{s}$ on the fluid to ensure the no-slip condition:

$$
\left.\mathbf{u}\right|_{\Gamma_{s}}=\mathbf{u}_{s} .
$$

In [18], the authors introduced a second-order accurate numerical scheme to enforce a Dirichlet boundary condition for the Poisson equation in irregular domains. We follow this approach to implicitly impose the no-slip condition when considering the viscous term, i.e. in the case of the linear systems for both GFCMI and GFSCMI given in section 3.3.2.

The projection on the $x$-direction of the stress tensor in the case of GFCMI gives:

$$
\left.\nabla \cdot(2 \mu \underline{\underline{\mathbf{D}}}) \cdot \mathbf{e}_{x}\right|_{i+\frac{1}{2}, j}=\left.\frac{\partial}{\partial x}\left(2 \mu \frac{\partial u}{\partial x}\right)\right|_{i+\frac{1}{2}, j}+\left.\frac{\partial}{\partial y}\left(\mu\left(\frac{\partial u}{\partial y}+\frac{\partial v}{\partial x}\right)\right)\right|_{i+\frac{1}{2}, j} .
$$

The discretization of equation (20) gives:

$$
\begin{aligned}
\left.\nabla \cdot(2 \mu \underline{\underline{\mathbf{D}}}) \cdot \mathbf{e}_{x}\right|_{i+\frac{1}{2}, j} & \frac{2 \mu_{i+1 j}\left(\frac{\partial u}{\partial x}\right)_{i+1, j}-2 \mu_{i, j}\left(\frac{\partial u}{\partial x}\right)_{i, j}}{\Delta x_{i}} \\
& +\frac{\mu_{i+\frac{1}{2} j+\frac{1}{2}}\left(\frac{\partial u}{\partial y}\right)_{i+\frac{1}{2}, j+\frac{1}{2}}-\mu_{i+\frac{1}{2} j-\frac{1}{2}}\left(\frac{\partial u}{\partial y}\right)_{i+\frac{1}{2}, j-\frac{1}{2}}}{\Delta y_{j-\frac{1}{2}}} \\
& +\frac{\mu_{i+\frac{1}{2} j+\frac{1}{2}}\left(\frac{\partial v}{\partial x}\right)_{i+\frac{1}{2}, j+\frac{1}{2}}-\mu_{i+\frac{1}{2} j-\frac{1}{2}}\left(\frac{\partial u}{\partial x}\right)_{i+\frac{1}{2}, j-\frac{1}{2}}}{\Delta y_{j-\frac{1}{2}}}
\end{aligned}
$$

Each term is discretized as follows:

- Discretization of $\left.\frac{\partial u}{\partial x}\right|_{i+1, j}$ : 
If $\phi_{i+\frac{1}{2}, j} \phi_{i+\frac{3}{2} j}>0$, the cell $\left[i+\frac{1}{2}, i+\frac{3}{2}\right] \times[j]$ is entirely covered with fluid. In this case, the approximation is:

$$
\left.\frac{\partial u}{\partial x}\right|_{i+1, j}=\frac{u_{i+\frac{3}{2} j}-u_{i+\frac{1}{2}, j}}{\Delta x_{i+1}} .
$$

If $\phi_{i+\frac{1}{2}, j} \phi_{i+\frac{3}{2} j} \leq 0$, there are two possible situations: either the cell point $\left(i+\frac{1}{2}, j\right)$ is in the solid domain, which means that $u_{i+\frac{1}{2}, j}=0$, or the cell point $\left(i+\frac{3}{2}, j\right)$ is in the solid domain. In this scenario, the method of [18] is applied, giving:

$$
\left.\frac{\partial u}{\partial x}\right|_{i+1, j}=\frac{u_{\Gamma}-u_{i+\frac{1}{2}, j}}{\theta \Delta x_{i+1}}
$$

with $u_{\Gamma}$ the boundary velocity condition and $\theta \Delta x_{i+1}$ the length fraction of the cell covered by the fluid, hence:

$$
\theta=\frac{\left|\phi_{i+\frac{1}{2}, j}\right|}{\left|\phi_{i+\frac{1}{2}, j}\right|+\left|\phi_{i+\frac{3}{2} j}\right|} .
$$

The interpolated length fraction is computed on a staggered cell point in order to keep second-order accuracy with:

$$
\phi_{i+\frac{1}{2}, j}=\frac{\phi_{i+1, j}+\phi_{i, j}}{2} \text { and } \quad \phi_{i+\frac{3}{2}, j}=\frac{\phi_{i+2, j}+\phi_{i+1, j}}{2} .
$$

- Discretization of $\left.\frac{\partial u}{\partial x}\right|_{i, j}$ :

If $\phi_{i+\frac{1}{2}, j} \phi_{i-\frac{1}{2}, j}>0$ the cell $\left[i-\frac{1}{2}, i+\frac{1}{2}\right] \times[j]$ is entirely covered with fluid. In this case, the approximation is:

$$
\left.\frac{\partial u}{\partial x}\right|_{i, j}=\frac{u_{i+\frac{1}{2}, j}-u_{i-\frac{1}{2}, j}}{\Delta x_{i}}
$$

If $\phi_{i+\frac{1}{2}, j} \phi_{i-\frac{1}{2}, j} \leq 0$,there are two possible situations: either the cell point $\left(i+\frac{1}{2}, j\right)$ is in the solid domain, which means that $u_{i+\frac{1}{2}, j}=0$, or the cell point $\left(i-\frac{1}{2}, j\right)$ is in the solid domain. In this case, the method of [18] is applied, giving:

$$
\left.\frac{\partial u}{\partial x}\right|_{i, j}=\frac{u_{i+\frac{1}{2}, j}-u_{\Gamma}}{\theta \Delta x_{i}}, \quad \text { with } \quad \theta=\frac{\left|\phi_{i+\frac{1}{2}, j}\right|}{\left|\phi_{i+\frac{1}{2}, j}\right|+\left|\phi_{i-\frac{1}{2}, j}\right|} .
$$

- Discretization of $\left.\frac{\partial u}{\partial y}\right|_{i+\frac{1}{2}, j+\frac{1}{2}}$ :

If $\phi_{i+\frac{1}{2}, j+1} \phi_{i+\frac{1}{2}, j}>0$, the cell $\left[i+\frac{1}{2}\right] \times[j, j+1]$ is entirely covered with fluid. In this case, the approximation is:

$$
\left.\frac{\partial u}{\partial y}\right|_{i+\frac{1}{2}, j+\frac{1}{2}}=\frac{u_{i+\frac{1}{2}, j+1}-u_{i+\frac{1}{2}, j}}{\Delta y_{j+\frac{1}{2}}} .
$$


If $\phi_{i+\frac{1}{2}, j+1} \phi_{i+\frac{1}{2}, j} \leq 0$, there are two possible situations: either the cell point $\left(i+\frac{1}{2}, j\right)$ is in the solid domain, which means that $u_{i+\frac{1}{2}, j}=0$, or the cell point $\left(i+\frac{1}{2}, j+1\right)$ is in the solid domain. In this case, the method of [18] is applied, giving:

$$
\left.\frac{\partial u}{\partial y}\right|_{i+\frac{1}{2}, j+\frac{1}{2}}=\frac{u_{\Gamma}-u_{i+\frac{1}{2}, j}}{\theta \Delta y_{j+\frac{1}{2}}}, \quad \text { with } \quad \theta=\frac{\left|\phi_{i+\frac{1}{2}, j}\right|}{\left|\phi_{i+\frac{1}{2}, j}\right|+\left|\phi_{i+\frac{1}{2}, j+1}\right|} .
$$

- Discretization of $\left.\frac{\partial u}{\partial y}\right|_{i+\frac{1}{2}, j-\frac{1}{2}}$ :

If $\phi_{i+\frac{1}{2}, j-1} \phi_{i+\frac{1}{2}, j}>0$, the cell $\left[i+\frac{1}{2}\right] \times[j, j-1]$ is entirely covered with fluid. In this case, the approximation is:

$$
\left.\frac{\partial u}{\partial y}\right|_{i+\frac{1}{2}, j-\frac{1}{2}}=\frac{u_{i+\frac{1}{2}, j}-u_{i+\frac{1}{2}, j-1}}{\Delta y_{j-\frac{1}{2}}} .
$$

If $\phi_{i+\frac{1}{2}, j-1} \phi_{i+\frac{1}{2}, j} \leq 0$, there are two possible situations: either the cell point $\left(i+\frac{1}{2}, j\right)$ is in the solid domain, which means that $u_{i+\frac{1}{2}, j}=0$, or the cell point $\left(i+\frac{1}{2}, j-1\right)$ is in the solid domain. In this case, the method of [18] is applied, giving:

$$
\left.\frac{\partial u}{\partial y}\right|_{i+\frac{1}{2}, j-\frac{1}{2}}=\frac{u_{i+\frac{1}{2}, j}-u_{\Gamma}}{\theta \Delta y_{j-\frac{1}{2}}}, \quad \text { with } \quad \theta=\frac{\left|\phi_{i+\frac{1}{2}, j}\right|}{\left|\phi_{i+\frac{1}{2}, j}\right|+\left|\phi_{i+\frac{1}{2}, j-1}\right|} .
$$

Let us now focus on the cross derivatives:

- Discretization of $\left.\frac{\partial v}{\partial x}\right|_{i+\frac{1}{2}, j+\frac{1}{2}}$ :

If $\phi_{i+1, j+\frac{1}{2}} \phi_{i, j+\frac{1}{2}}>0$, the cell $[i, i+1] \times\left[j+\frac{1}{2}\right]$ is entirely covered with fluid. In this case, the approximation is:

$$
\left.\frac{\partial v}{\partial x}\right|_{i+\frac{1}{2}, j+\frac{1}{2}}=\frac{v_{i+1, j+\frac{1}{2}}-v_{i, j+\frac{1}{2}}}{\Delta x_{i+\frac{1}{2}}}
$$

If $\phi_{i+1, j+\frac{1}{2}} \phi_{i, j+\frac{1}{2}} \leq 0$, there are two possible situations: either the cell point $\left(i+1, j+\frac{1}{2}\right)$ is in the solid domain. In this case, the method of [18] is applied, giving:

$$
\left.\frac{\partial v}{\partial x}\right|_{i+\frac{1}{2}, j+\frac{1}{2}}=\frac{v_{\Gamma}-v_{i, j+\frac{1}{2}}}{\theta \Delta x_{i+\frac{1}{2}}}, \quad \text { with } \quad \theta=\frac{\left|\phi_{i, j+\frac{1}{2}}\right|}{\left|\phi_{i+1, j+\frac{1}{2}}\right|+\left|\phi_{i, j+\frac{1}{2}}\right|} .
$$

or the cell point $\left(i, j+\frac{1}{2}\right)$ is in the solid domain and:

$$
\left.\frac{\partial v}{\partial x}\right|_{i+\frac{1}{2}, j+\frac{1}{2}}=\frac{v_{i+1, j+\frac{1}{2}}-v_{\Gamma}}{\theta \Delta x_{i+\frac{1}{2}}}, \quad \text { with } \quad \theta=\frac{\left|\phi_{i+1, j+\frac{1}{2}}\right|}{\left|\phi_{i+1, j+\frac{1}{2}}\right|+\left|\phi_{i, j+\frac{1}{2}}\right|} .
$$

- Discretization of $\left.\frac{\partial v}{\partial x}\right|_{i+\frac{1}{2}, j-\frac{1}{2}}$ : 
If $\phi_{i+1, j-\frac{1}{2}} \phi_{i, j-\frac{1}{2}}>0$, the cell $[i, i+1] \times\left[j-\frac{1}{2}\right]$ is entirely covered with fluid. In this case, the approximation is:

$$
\left.\frac{\partial v}{\partial x}\right|_{i+\frac{1}{2}, j-\frac{1}{2}}=\frac{v_{i+1, j-\frac{1}{2}}-v_{i, j-\frac{1}{2}}}{\Delta x_{i+\frac{1}{2}}} .
$$

If $\phi_{i+1, j-\frac{1}{2}} \phi_{i, j-\frac{1}{2}} \leq 0$, there are two possible situations: either the cell point $\left(i+1, j-\frac{1}{2}\right)$ is in the solid domain. In this case, the method of [18] is applied, giving:

$$
\left.\frac{\partial v}{\partial x}\right|_{i+\frac{1}{2}, j-\frac{1}{2}}=\frac{v_{\Gamma}-v_{i, j-\frac{1}{2}}}{\theta \Delta x_{i+\frac{1}{2}}}, \quad \text { with } \quad \theta=\frac{\left|\phi_{i, j-\frac{1}{2}}\right|}{\left|\phi_{i+1, j-\frac{1}{2}}\right|+\left|\phi_{i, j-\frac{1}{2}}\right|} .
$$

or the cell point $\left(i, j-\frac{1}{2}\right)$ is in the solid domain and:

$$
\left.\frac{\partial v}{\partial x}\right|_{i+\frac{1}{2}, j-\frac{1}{2}}=\frac{v_{i+1, j-\frac{1}{2}}-v_{\Gamma}}{\theta \Delta x_{i+\frac{1}{2}}}, \quad \text { with } \quad \theta=\frac{\left|\phi_{i+1, j-\frac{1}{2}}\right|}{\left|\phi_{i+1, j-\frac{1}{2}}\right|+\left|\phi_{i, j-\frac{1}{2}}\right|} .
$$

The procedure is easier for GFSCMI, as the cross derivative terms are considered explicitly as a jump condition in the pressure resolution instead of implicitly.

\subsection{Imposing the Contact Angle}

When both the liquid-gas interface and the fluid-solid interfaces are located in the same cell, one needs to compute the surface tension effect on the irregular domain boundaries. Modeling accurately the contact line on a wall is challenging because the surface physics is not currently completely understood. In this study we only focus on static contact angles based on thermodynamic properties. Modeling dynamic contact angles is out of the scope of this paper for the reasons that are provided in section 3.6.

When the equilibrium state is reached, the static contact angle $\theta_{s}$ (see figure 1) can be used to obtain a Neumann boundary condition on the fluid interface level-set function:

$$
\mathbf{n}_{s} \cdot \nabla \phi=\cos \theta_{s} \quad \text { with } \quad \mathbf{n}_{s}=\frac{\nabla \phi_{s}}{\left|\nabla \phi_{s}\right|}
$$

As $\theta_{s}$ is known from the properties of the flow, one needs to enforce this condition on $\phi$ when a contact line appears. Our method is based on the multidimensional extrapolation suggested by $[3,15]$ to define the fluid level-set function's extrapolation in the irregular domain:

$$
\frac{\partial \phi}{\partial \tau}+H\left(\phi_{s}\right) \mathbf{n}_{s} \cdot \nabla \phi=\cos \theta_{s},
$$

where $\tau$ is a fictitious time step, and $H\left(\phi_{s}\right)$ a Heaviside function. Equation (21) is only applied on the cells in solid domains. A second order upwind scheme was used for the discretization of $\mathbf{n}_{s} \cdot \nabla \phi$.

The curvature also needs to be extrapolated to properly handle the pressure jump condition in cells where the three phases are present. The extrapolation process is the same that the one used for the level-set function in equation (22), i.e.:

$$
\frac{\partial \kappa}{\partial \tau}+H\left(\phi_{s}\right) \mathbf{n}_{s} \cdot \nabla \kappa=0,
$$


where we recall that $\kappa$ is the local mean curvature.

We note that this procedure is similar but different from that of [28], in which the authors consider a moving contact line with a reconstruction of the level-set function up to $2.5 \Delta x$ in the fluid domain in order to keep a smooth computation of the local curvature. This procedure thus alters the flows near the solid-fluid interface. In our computation of the static contact angle, the extension of the local curvature is in the solid media only and does not affect the flow.

\subsection{Advancing and Receding Contact Line}

It is well known that the contact angle of a droplet or a bubble can vary when they move on a solid surface. In particular, it has been observed in several experimental studies that the value of the contact angle depends on the interface velocity and on its sign, i.e. the contact angle will take different value depending on whether the interface is advancing or receding, all other physical parameters being equal. Moreover, the dynamics of the interface will not only depend on the physicochemical properties of the fluid and of the solid, but also on the surface quality, which can be characterized for example by its roughness. De Gennes showed that roughness can induce a hysteresis phenomenon on the contact angle [17]. In [45], the author presents simulations involving contact angle hysteresis enforced on a solid boundary fitted to the computational domain.

Although many studies have been performed on this difficult topic $[1,43,44,45,46]$, there is a lack of consensus on how to handle a moving contact angle in numerical simulations in a way that does not depend on the grid cell size. For this reason, we do not propose in this paper any numerical models to account for a moving contact line. Indeed, this topic is out of the scope of this paper, since this problem should be solved accurately on simplest configurations without irregular domains (horizontal wall fitted to the computational domain) before performing an implementation with irregular domains. Consequently, the validations of our numerical simulations will involve configurations with a steady shape of the interface. We note, however, that considering a static contact line enables us to illustrate the extent to which the method limits spurious parasitic currents and thus illustrate its numerical stability.

\section{Numerical Examples and Validations}

In this section, we first provide numerical examples that test the two different viscosity solvers (GFCMI and GFSCMI). Because of the lack of analytical solutions for two-phase flows, we consider one-phase flows. We then turn our attention to two-phase flows and report numerical results in two and three spatial dimensions.

\subsection{Flow Past a Cylinder}

We consider a flow past a cylinder in order to show that the different discretizations are able to compute both steady and unsteady regimes. Coutanceau and Bouard [12] showed in an experiment that a transition regime exists between Reynolds numbers 40 and 50. Consider a domain $\Omega=$ $[-16 ; 16] \times[-8 ; 8]$ and a cylinder with a radius of $r=0.5$ and center at $[0,0]$. We impose a Dirichlet condition $U_{\infty}=1 \mathrm{~m} \cdot \mathrm{s}^{-1}$ on the left, symmetric boundary conditions on the top and bottom, and an outflow boundary condition on the right of the domain, with a no-slip condition on the cylinder boundary. In these simulations we define the viscosity coefficient as $\mu=2 r U_{\infty} / R e$, with $R e=20,40$ and 100, corresponding respectively to the case A, B and C. Experimental and 
numerical experiments from others allow us to compare the total drag, $C_{D}$, and lift, $C_{L}$, forces acting on the cylinder:

$$
\mathbf{F}_{f \rightarrow s}=\int_{\theta=0}^{2 \pi} \underline{\underline{\sigma}} \mathbf{n} R d \theta \quad \text { with } \quad \underline{\underline{\sigma}}=-p \mathbf{I I}+2 \mu \underline{\underline{\mathbf{D}}},
$$

giving the drag and lift coefficients:

$$
C_{D}=\frac{F_{x}}{\frac{1}{2} \rho U_{\infty}^{2} D} \quad \text { and } \quad C_{L}=\frac{F_{y}}{\frac{1}{2} \rho U_{\infty}^{2} D} .
$$

For steady flows, one can also measure some characteristics of the vortices in the wake of the cylinder, such as their centers position $\left(x_{c}, y_{c}\right)$ and the length, $x_{s}$, of the recirculation as illustrated in Fig 3.

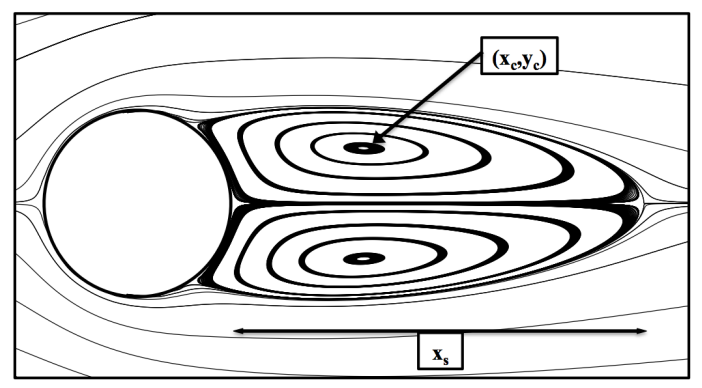

Figure 3: Streamlines for a flow past a cylinder at $R e=40$. Characteristics such as the location of the vortices center $\left(x_{c}, y_{c}\right)$ and the length, $x_{s}$, of the recirculation are represented.

We will present here some comparisons for a one-phase flow between the Implicit Conservative Viscous Method (ICVM) and the Implicit Primitive Viscous Method (IPVM) which corresponds respectively to GFCMI and to GFSCMI for a two-phase flow. Indeed, most studies involving onephase flows use a numerical scheme similar to the IPVM [34] to deal with viscous terms. However as it has been discussed previously, in the framework of two-phase flows, a numerical scheme based on a conservative formulation of the viscous terms can be preferred for temporal stability and accuracy (see Appendix for more details).

Therefore, this test-case will allow us to check that both methods will provide similar results in order to validate the ICVM for a one-phase flow before performing computations of GFCMI in irregular domains. The simulations have been performed with four different grids, $128 \times 256$, $256 \times 512,512 \times 1024$, and $1024 \times 2048$, which correspond respectively to $\Delta x=D / 8, \Delta x=D / 16$, $\Delta x=D / 32$ and $\Delta x=D / 64$.

Streamlines and vorticity contours have been plotted in figure 4 for cases $A$ and $B$ corresponding to $R e=20$ and $R e=40$, respectively. Steady vortices are attached to the rear of the cylinder. These vortices are growing if the Reynolds number is increased. In table 1, some comparisons between our numerical simulations and several numerical and experimental studies are presented. Our two schemes (ICVM and IPVM) provide similar results and converge to an expected value if the size of the grid cell is at least equal to $\Delta x=D / 16$.

In the case $R e=100$, the flow in the wake of the cylinder is unsteady due to the development of a Von-Kármán instability. The vorticity contours have been plotted in figure 5 in order to visualize 

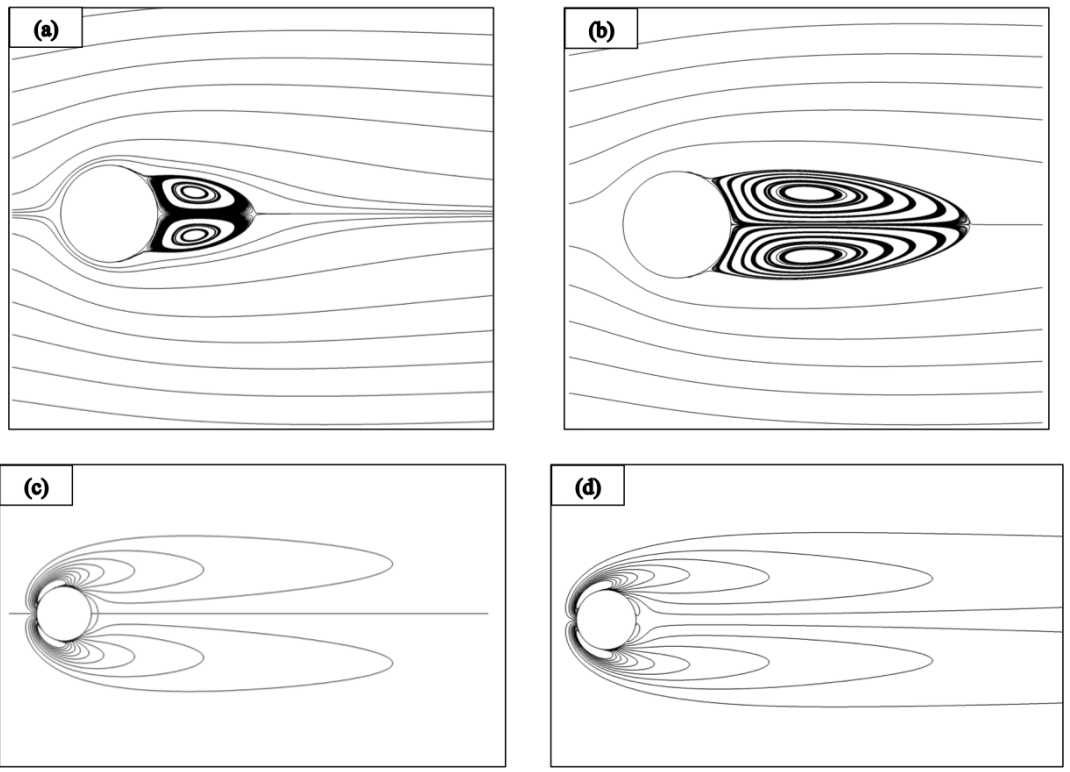

Figure 4: Streamline representation and vorticity contours $\left(s^{-1}\right)[-5 ; .5 ; 5]$ for Case A $(\mathrm{a}, \mathrm{c})$ and B (b,d).

\begin{tabular}{|c||c|c|c|c||c|c|c|c|}
\hline \multicolumn{1}{|c||}{} & \multicolumn{4}{c||}{$\mathrm{Re}=20$} & \multicolumn{5}{c|}{$\mathrm{Re}=40$} \\
& $C_{D}$ & $x_{s}$ & $x_{c}$ & $y_{c}$ & $C_{D}$ & $x_{s}$ & $x_{c}$ & $y_{c}$ \\
\hline Coutanceau \& Bouard [12] & - & 0.93 & 0.33 & 0.23 & - & 2.13 & 0.76 & 0.29 \\
\hline Xu [60] & 2.23 & 0.93 & 0.36 & 0.21 & 1.66 & 2.24 & 0.72 & 0.3 \\
\hline Ye et al. $[62]$ & 2.03 & 0.92 & - & - & 1.52 & 2.27 & - & - \\
\hline Xie \& Desjardin [58] & 2.19 & 1 & - & - & 1.60 & 2.26 & - & - \\
\hline Tritton [53] & 2.22 & - & - & - & 1.48 & - & - & - \\
\hline Present study with ICVM & & & & & & & & \\
$\Delta x=D / 8$ & 2.25 & 0.9 & 0.318 & 0.217 & 1.64 & 2.24 & 0.714 & 0.297 \\
$\Delta x=D / 16$ & 2.214 & 0.942 & 0.344 & 0.223 & 1.628 & 2.251 & 0.709 & 0.299 \\
$\Delta x=D / 32$ & 2.224 & 0.937 & 0.351 & 0.218 & 1.614 & 2.227 & 0.706 & 0.297 \\
$\Delta x=D / 64$ & 2.234 & 0.933 & 0.353 & 0.217 & 1.621 & 2.221 & 0.707 & 0.297 \\
\hline Present study with IPVM & & & & & & & & \\
$\Delta x=D / 8$ & 2.318 & 0.916 & 0.317 & 0.221 & 1.687 & 2.255 & 0.713 & 0.3 \\
$\Delta x=D / 16$ & 2.239 & 0.946 & 0.344 & 0.224 & 1.639 & 2.257 & 0.709 & 0.3 \\
$\Delta x=D / 32$ & 2.247 & 0.94 & 0.351 & 0.218 & 1.625 & 2.228 & 0.706 & 0.298 \\
$\Delta x=D / 64$ & 2.250 & 0.933 & 0.352 & 0.217 & 1.628 & 2.220 & 0.707 & 0.297 \\
\hline
\end{tabular}

Table 1: Drag coefficient and recirculations characteristics for $R e=20$ and $R e=40$.

the periodic detachment of vortices in the wake of the cylinder. The temporal evolution of the drag and the lift coefficients are also presented in figure 5. Quantitative comparisons between our numerical simulations and other data from the literature are reported in table 2 . These comparisons show a satisfactory agreement between all results. 

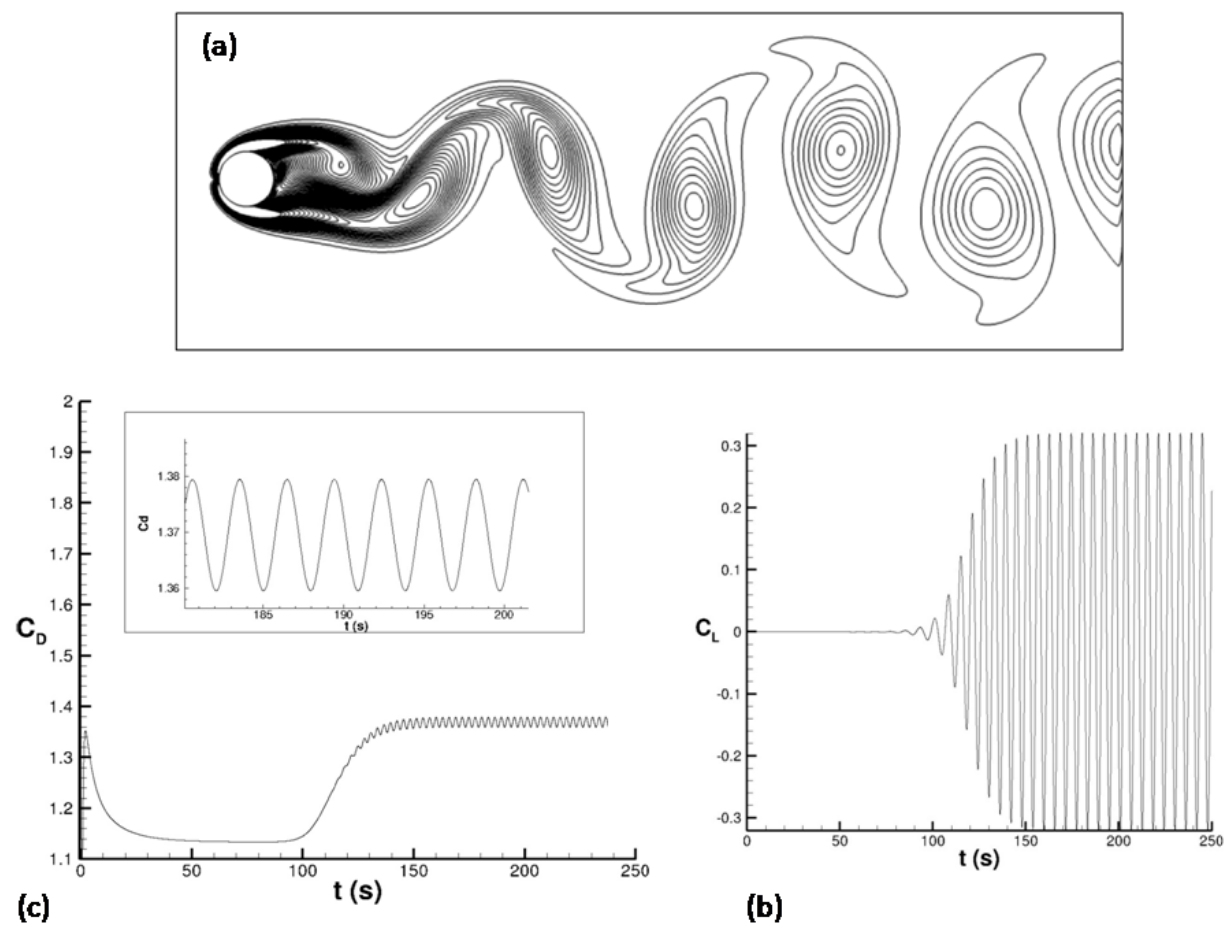

(b)

Figure 5: (a) Vorticity contours $[-4: .15: 4]$ for Case C. (b) and (c) represent the evolution of the drag and lift coefficients through time.

The configurations studied in this case do not allow a proper convergence study, as no theoretical result exists . In [16], the authors suggest a convergence study at early flow times, based on the 'best converged results'. Figure 6 presents our results for Reynolds numbers 20, 40 and 100, where the error is computed as $e=\left|r_{b c}-r_{g m}\right|$, with $r_{b c}$ the best converged result and $r_{g m}$ the result for a given mesh. The spatial convergence for early flow times observed in figure 6 shows a satisfactory agreement with the previous study made by Gibou et al. in [34] on analytical cases where the $2^{\text {nd }}$ spatial order was proved for the ICVM.

We present in figure 7 the temporal coefficient for two given grids, respectively $\Delta x=D / 32$ and $\Delta x=D / 64$, with the ICVM discretization of the viscous terms. Indeed, it has been reported in [41] that the dimensionless time where the instability starts for $R e=100$ depends on the numerical methods which are used, see for example figure 6.b in the referred paper. Our simulations bring out that the duration of the transient regime can also depend slightly on the mesh refinement, with respectively $T_{\text {adim }}=182$ and $T_{\text {adim }}=166$ for the two successive meshes. These durations are close to the results reported in [41].

\subsection{Flow Past a Sphere}

We now consider the simulation of the flow past a sphere. This study will allow us to show that our numerical simulations are able to reproduce 3D steady axisymmetric and 3D non-axisymmetric 


\begin{tabular}{|c||c|c|c|}
\hline \multicolumn{1}{|c||}{} & \multicolumn{3}{c|}{$R e=100$} \\
& $C_{L}$ & $C_{D}$ & $S t=f D / U_{\infty}$ \\
\hline Berthelsen [4] et al. & \pm 0.34 & $1.38 \pm 0.01$ & 0.169 \\
\hline Braza [7] et al. & \pm 0.25 & $1.346 \pm 0.015$ & - \\
\hline Liu [28] et al. & \pm 0.339 & $1.35 \pm 0.012$ & - \\
\hline Calhoun [9] & \pm 0.298 & $1.330 \pm 0.014$ & 0.175 \\
\hline Ng et al. $[34]$ & \pm 0.36 & $1.368 \pm 0.016$ & - \\
\hline Guittet et al. $[20]$ & \pm 0.331 & $1.401 \pm 0.016$ & - \\
\hline Xu [59] et al. & \pm 0.353 & $1.42 \pm 0.013$ & 0.171 \\
\hline Choi [10] et al. & \pm 0.315 & $1.4 \pm 0.011$ & - \\
\hline Present study with ICVM & & & \\
$\Delta x=D / 8$ & \pm 0.261 & $1.404 \pm 0.008$ & 0.167 \\
$\Delta x=D / 16$ & \pm 0.334 & $1.419 \pm 0.011$ & 0.170 \\
$\Delta x=D / 32$ & \pm 0.321 & $1.369 \pm 0.010$ & 0.170 \\
$\Delta x=D / 64$ & \pm 0.321 & $1.369 \pm 0.010$ & 0.170 \\
\hline Present study with IPVM & & & \\
$\Delta x=D / 8$ & \pm 0.262 & $1.430 \pm 0.008$ & 0.167 \\
$\Delta x=D / 16$ & \pm 0.336 & $1.424 \pm 0.011$ & 0.168 \\
$\Delta x=D / 32$ & \pm 0.322 & $1.376 \pm 0.010$ & 0.169 \\
$\Delta x=D / 64$ & \pm 0.322 & $1.371 \pm 0.010$ & 0.169 \\
\hline
\end{tabular}

Table 2: Drag and lift coefficients and Strouhal number for $R e=100$ for a flow past a cylinder.

steady flows. Consider a domain $\Omega=[-8,8] \times[-4,4] \times[-4,4]$ and a sphere with radius $r=0.5$ and center located at $(0,0)$. We impose a Dirichlet condition $U_{\infty}=1 \mathrm{~m} \cdot \mathrm{s}^{-1}$ on the left, symmetric conditions on the top, bottom, front and back walls, and an outflow boundary condition on the right side of the computational field. A no-slip boundary condition is imposed on the sphere's boundary. In these simulations, we define the viscosity coefficient as $\mu=2 r U_{\infty} / R e$, with $R e=$ $25,50,100,150$ and 200 for steady axisymmetric flows as depicted by figure 8 , and 250 for the steady non-axisymmetric flow.

The drag and lift coefficients are defined as:

$$
\begin{gathered}
\mathbf{F}_{f \rightarrow s}=\int_{\theta=0}^{\pi} \int_{\Psi=0}^{2 \pi} R^{2} \sin \theta \underline{\underline{\sigma}} \mathbf{n} d \theta d \Psi \\
C_{D}=\frac{F_{x}}{\frac{1}{2} \rho U_{\infty}^{2} \pi D^{2} / 4} \quad \text { and } \quad C_{L}=\frac{F_{y}}{\frac{1}{2} \rho U_{\infty}^{2} \pi D^{2} / 4} .
\end{gathered}
$$

Figure 9 gives some comparisons on the drag coefficient obtained from several published works, including the experimental studies of $[22,40]$, numerical simulations, and the semi-empiric correlation of Schiller-Naumann. This correlation is given by the following relation:

$$
C_{D}=\left\{\begin{array}{cl}
\frac{24\left(1+0.15 R e^{0.687}\right)}{R e} & \text { for } R e \leq 1000 \\
0.44 & \text { for } R e>1000
\end{array}\right.
$$

Simulations for both IPVM and ICVM are presented for $\Delta x=D / 16$ which corresponds to a grid with $128^{3}$ cells. We can observe a good agreement of our numerical simulations with the results 


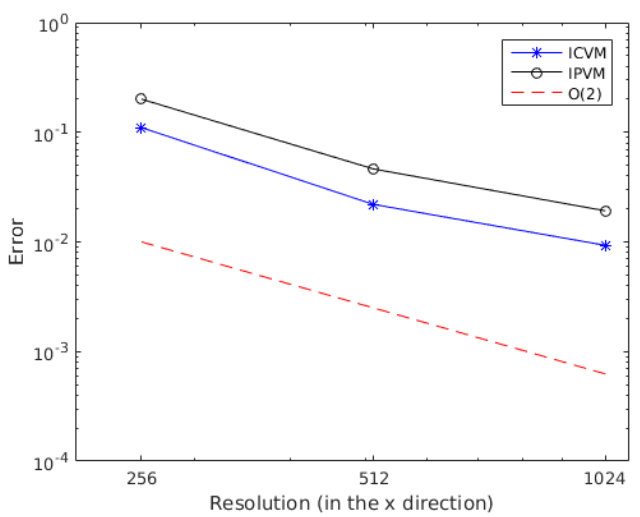

(a) $R e=20$

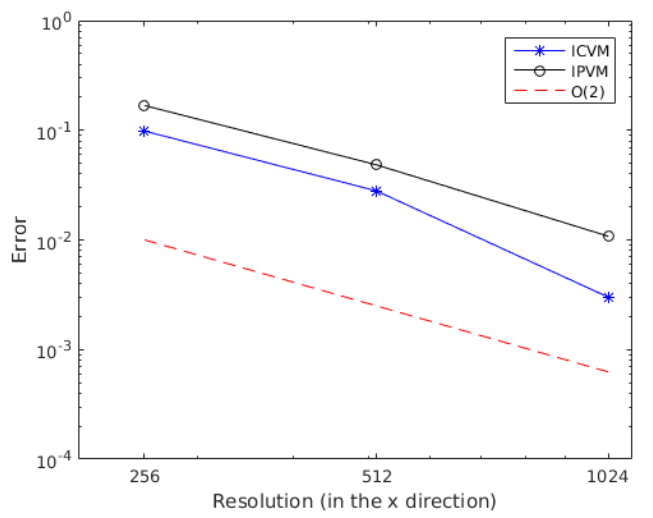

(b) $R e=40$

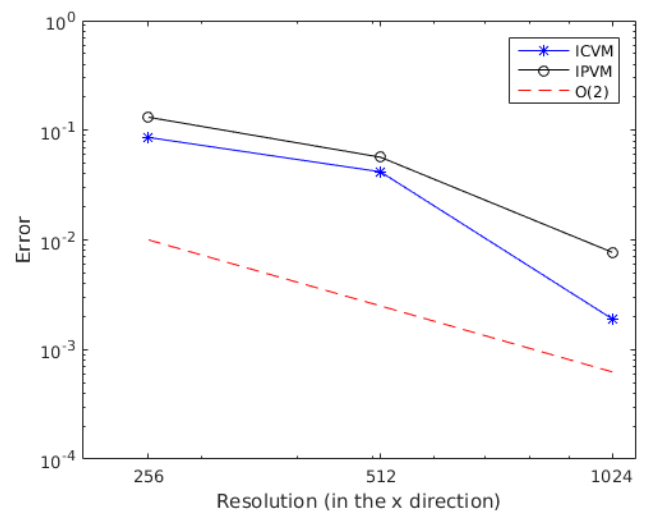

(c) $R e=100$

Figure 6: Convergence at early flow time for a flow past a cylinder at Re 20, 40 and 100.

\begin{tabular}{|c||c|c|c|c|c|}
\hline & $R e=25$ & $R e=50$ & $R e=100$ & $R e=150$ & $R e=200$ \\
\hline Schiller-Naumann & 2.27 & 1.53 & 1.09 & 0.91 & 0.80 \\
\hline $\begin{array}{c}\text { ICVM Method } \\
\Delta x=D / 8\end{array}$ & 2.27 & 1.49 & 1.051 & 0.88 & 0.78 \\
\hline$\Delta x=D / 16$ & 2.23 & 1.464 & 1.017 & 0.84 & 0.74 \\
\hline $\begin{array}{c}\text { IPVM Method } \\
\Delta x=D / 8\end{array}$ & 2.31 & 1.50 & 1.052 & 0.87 & 0.77 \\
\hline$\Delta x=D / 16$ & 2.268 & 1.48 & 1.024 & 0.84 & 0.74 \\
\hline
\end{tabular}

Table 3: Convergence of drag coefficient for a flow past a sphere, for ICVM and IPVM for different Reynolds numbers in the steady regime.

reported in the literature. In table 3, we present some comparisons of the drag coefficient between our simulations for two grid resolutions and the Schiller-Naumann law. These comparisons show 


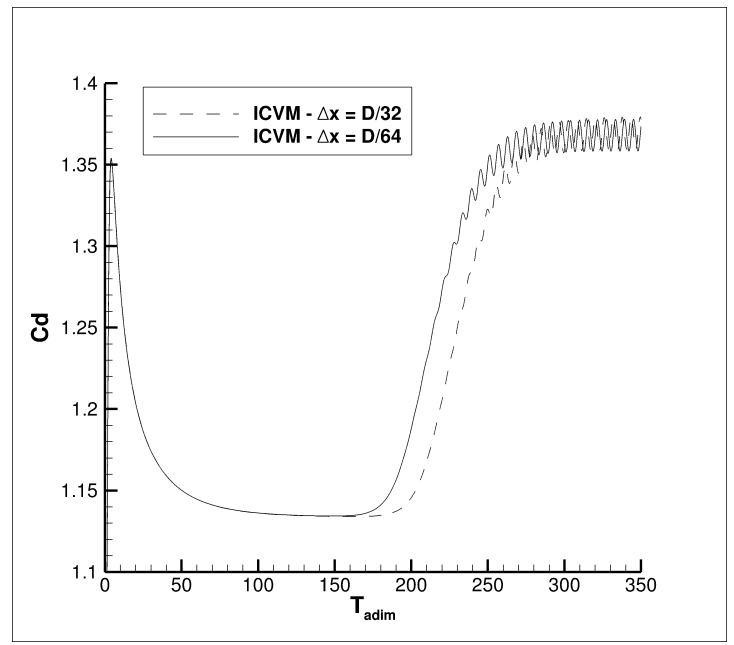

Figure 7: Drag coefficients for two successive meshes with the ICVM versus $T_{\text {adim }}=2 t D / U_{\infty}$.

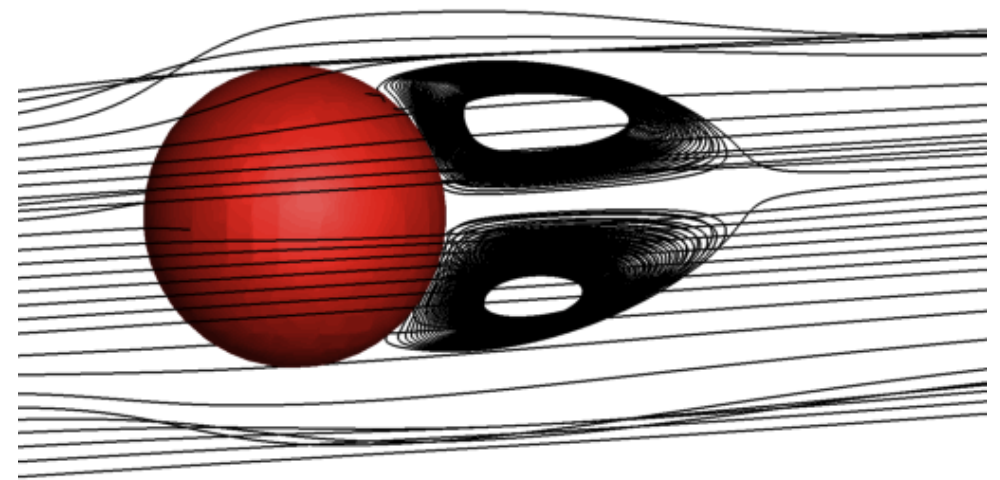

Figure 8: Illustration of streamlines for a flow past a sphere at $R e=150$.

that the two numerical methods converge towards the same solutions with a slight discrepancy $(<10 \%)$ with the Schiller-Naumann correlation.

Figure 10 presents a comparison of the recirculation length as well as the position of the center of the vortices between our simulation and the experimental results from Johnson \& Patel [22], Taneda [49], Magnaudet [29]. In this case, we use the ICVM method with $\Delta x=D / 16$. One can observe good agreement. Figure 11 compares the streamlines and vorticity contours from Johnson and Patel [22] and our simulations for $R e=150$.

Figure 12 compares the streamlines results obtained with our simulations and those of Johnson and Patel [22] for $R e=250$. We can observe the expected instability and that the streamlines are in good agreement. Figure 13 presents some projected streamlines on different planes. The qualitative comparison for this non-axisymmetric case are in good agreement with [22] for both IPVM and ICVM. In conclusion, one can observe that IPVM and ICVM behave similarly in terms of numerical accuracy. 


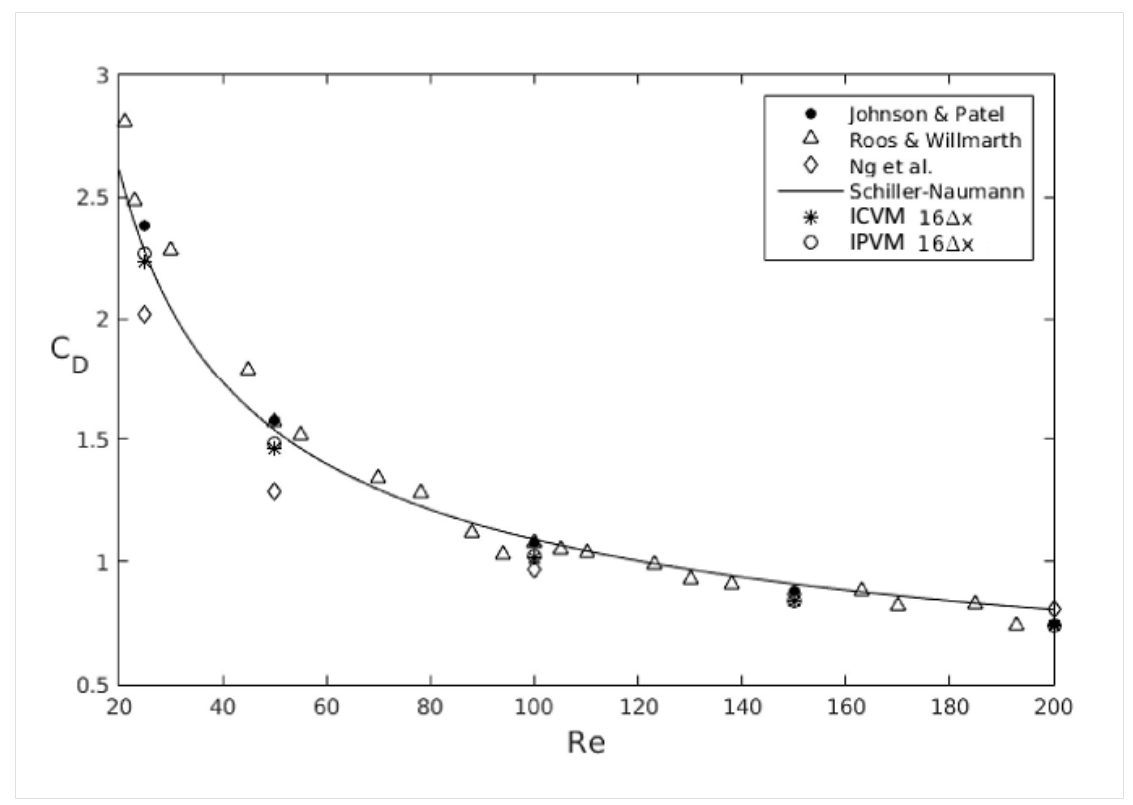

Figure 9: Drag coefficient evolution versus the Reynolds number.

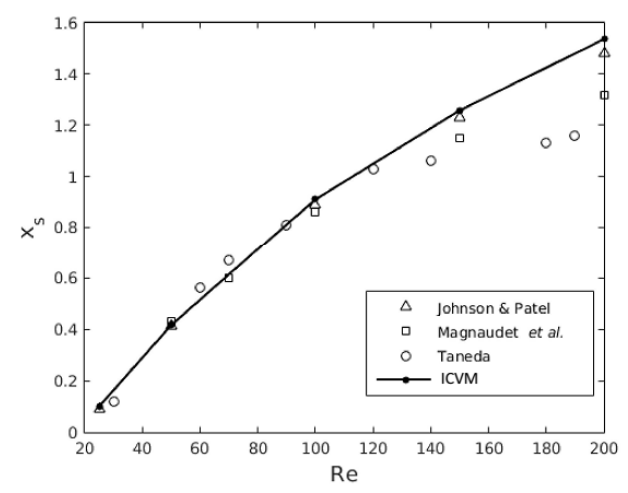

(a) Recirculation length

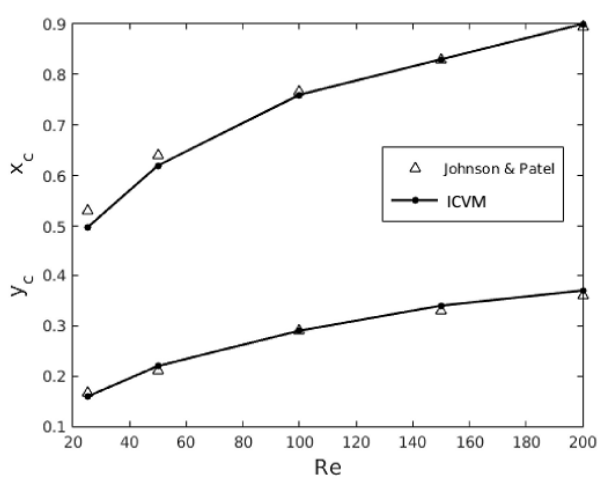

(b) Position of the vortices center

Figure 10: Geometric characteristics of the flow past a sphere for different Reynolds number

\subsection{Two Dimensional Two-Phase Flows in Irregular Domains without Volume Forces}

In this section, we present some numerical simulations of a hemispherical droplet deposited on a wall without initial velocity. These simulations are performed with a horizontal solid boundary (aligned to mesh axes) but also with a slanted plane, see figure 14, in order to demonstrate the ability of the proposed numerical method to obtain the expected shape of the droplet deposited on arbitrary solid boundaries. This configuration is instigated by the previous work of Dupont [14] which has been performed with a horizontal solid boundary located on the boundary of the 

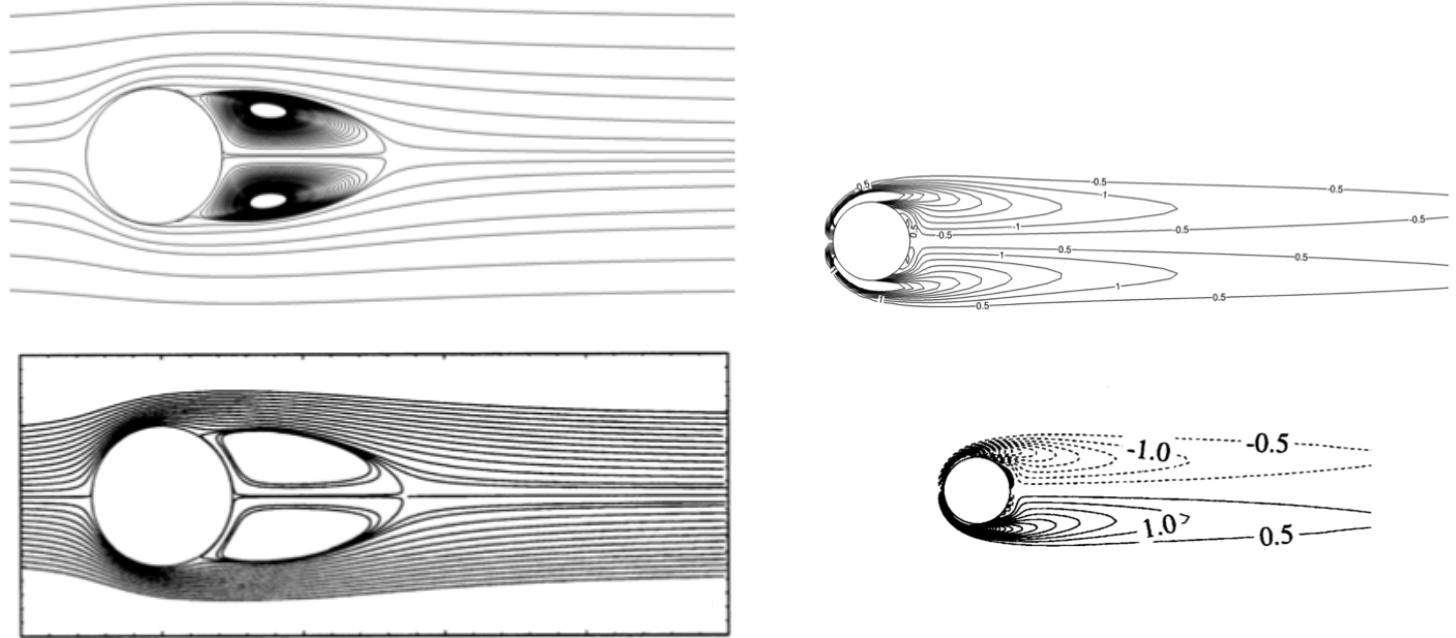

Figure 11: Top: streamlines (left) and vorticity (right) contours [-5:.5:5] in the $x-y$ plane for $R e=150$. Bottom: corresponding results from Johnson \& Patel [22].

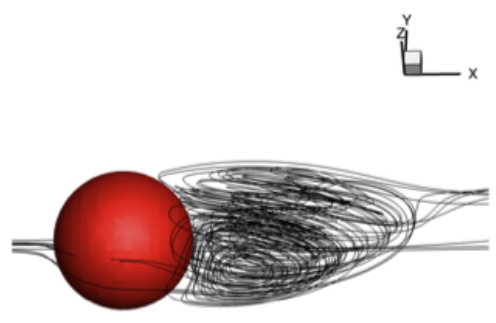

(a)
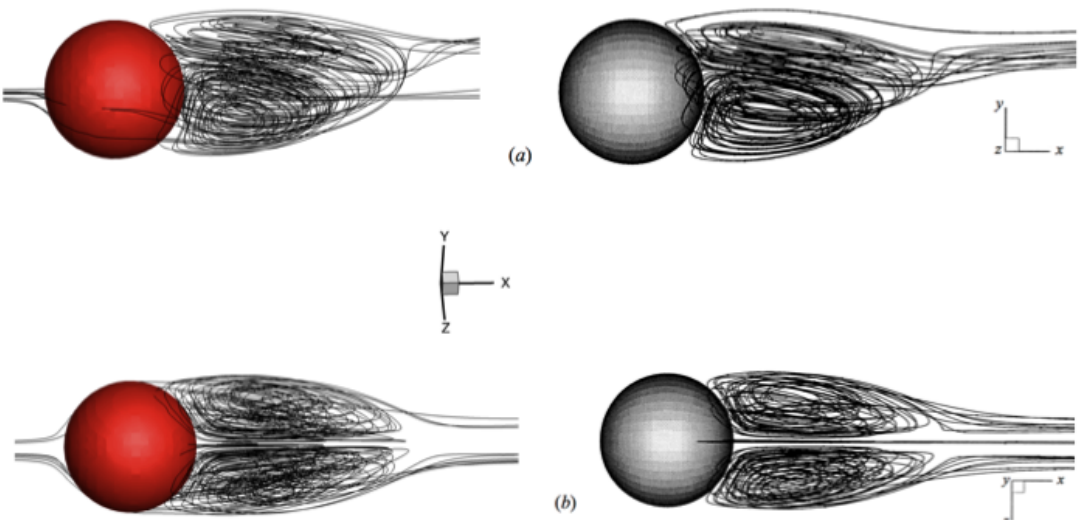

(b)

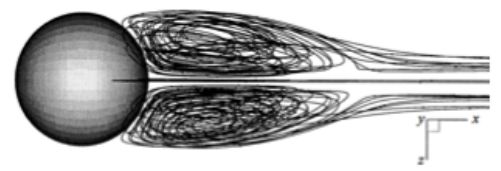

Figure 12: Illustration of streamlines for a flow past a sphere at $R e=250$. On the left present results and on the right corresponding results from [22].

computational domain. An additional difficulty suggested in [35] is to consider embedded solid boundary instead of using the computational domain as solid boundaries. If the static contact 

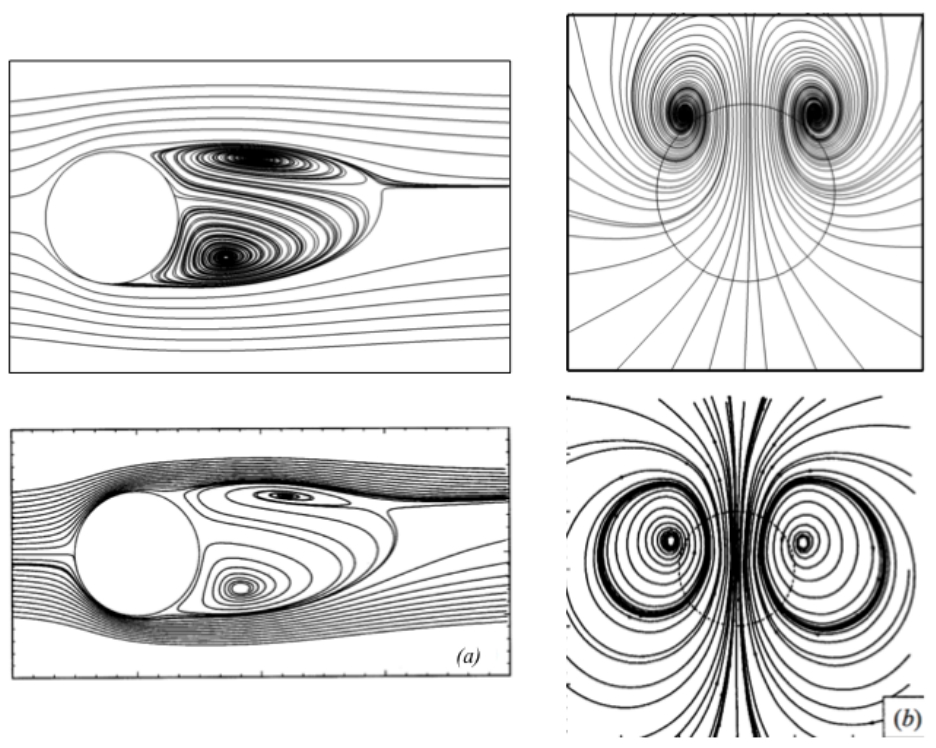

Figure 13: Illustration of streamlines for a flow past a sphere at $R e=250$, on top our simulation and bottom corresponding results from [22]. (a) Streamlines projected on the on the x-y plane for [22], and (b) streamlines projected on the $\mathrm{y}-\mathrm{z}$ plane 8 diameters downstream from the sphere center.

angle is not $90^{\circ}$ the contact line will move until equilibrium is reached. If the gravity is zero, the exact final shape of the drop can be obtained due to geometrical considerations and mass conservation.

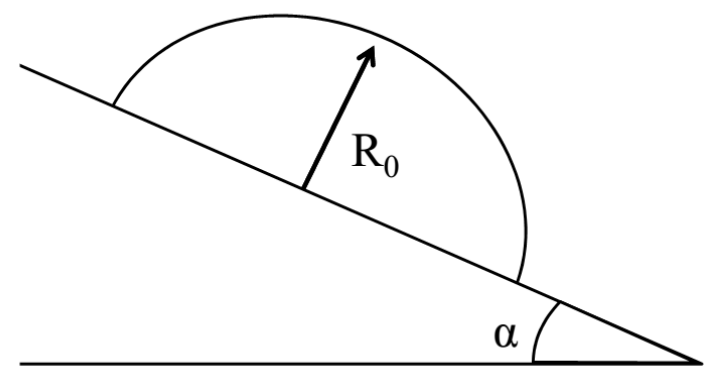

Figure 14: Initial shape of the drop released on an slanted plan.

The numerical domain is $\Omega=\left[-3 R_{0}, 3 R_{0}\right] \times\left[-3 R_{0}, 3 R_{0}\right]$. The simulations are carried out with both GFSCMI and GFCMI, with a horizontal wall, and with a slanted wall (for $\alpha=40^{\circ}$ ), with $\theta_{s} \in[30,120]$, and the following parameters: $R_{0}=6.4 \times 10^{-4} \mathrm{~m}, \sigma=7.2 \times 10^{-2} \mathrm{~N} \cdot \mathrm{m}^{-1}$, $\rho_{L}=1000 \mathrm{~kg} \cdot \mathrm{m}^{-3}$ and $\mu_{L}=10^{-2} \mathrm{~kg} \cdot \mathrm{m}^{-1} \cdot \mathrm{s}^{-1}$ for the liquid and $\rho_{G}=1 \mathrm{~kg} \cdot \mathrm{m}^{-3}$ and $\mu_{G}=$ $10^{-5} \mathrm{~kg} \cdot \mathrm{m}^{-1} \cdot \mathrm{s}^{-1}$ for the gas. 
For 2D cases without volume forces, mass conservation gives:

$$
R=R_{0} \sqrt{\frac{\pi}{2\left(\theta_{s}-\sin \theta_{s} \cos \theta_{s}\right)}}, \quad L=2 R \sin \theta_{s}, \quad e=R\left(1-\cos \theta_{s}\right),
$$

with $R$ the equilibrium radius of the circle, $L$ the spreading length, $e$ the height of the drop, as illustrated in figure 15 .

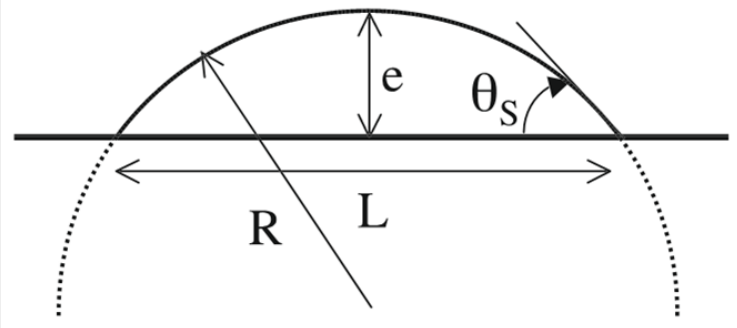

Figure 15: Characteristics of the final drop shape (from [14]).

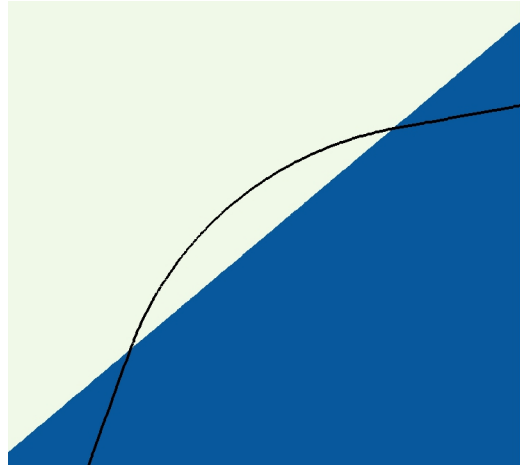

(a) $\theta_{s}=30^{\circ}$

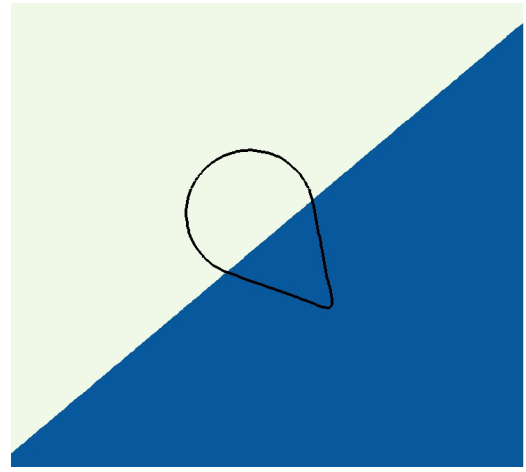

(b) $\theta_{s}=120^{\circ}$

Figure 16: Shapes of 2D drops on a slanted wall for different values of the contact angle.

Figure 16 illustrates the shapes of two drops at equilibrium on a slanted wall for two static contact angles, $\theta_{s}=30^{\circ}$ and $\theta_{s}=120^{\circ}$. In figure 17 and figure 18 , one can observe the convergence to expected theoretical values. Figure 17 gives the results for a wall aligned to the mesh, while figure 18 shows non-aligned wall to the mesh. Both methods converge towards the correct solution, with a gain in accuracy is observed with GFCMI. In order to compare more accurately the two methods, a study on spurious currents and mass conservation for different static contact angles is presented in table 4 .

In order to quantify the accuracy of the numerical methods, we have performed a mesh sensitivity study with three different grids for different contact angles in the case of a slanted wall. The results are presented in table 4 , where the value of $e, L,\left|V_{\max }\right|$ and the volume loss are reported in the case of GFCMI and GFSCMI. The maximum velocity is reported when the drop reaches its 


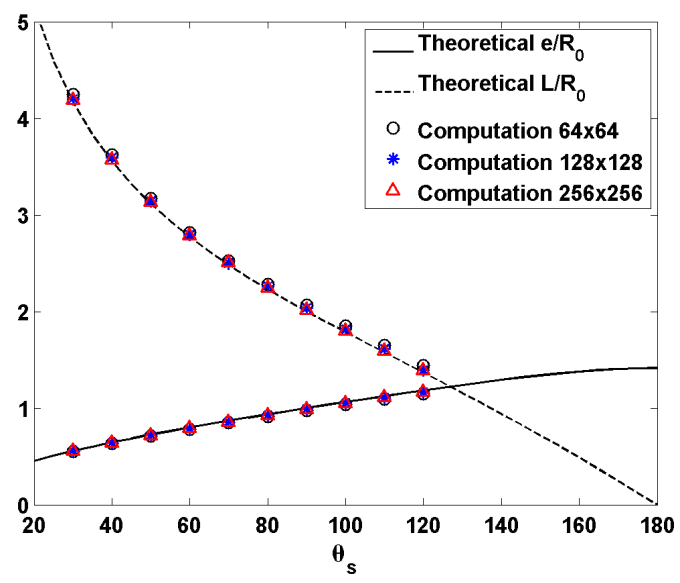

(a) GFCMI

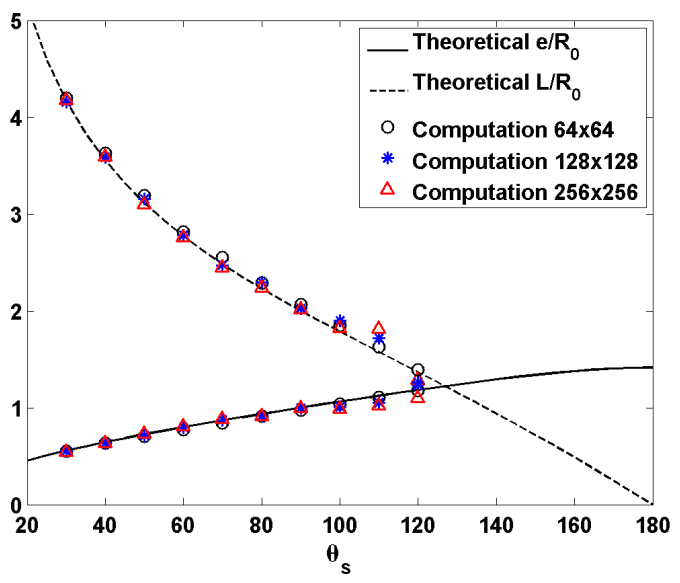

(b) GFSCMI

Figure 17: Results on a flat wall.

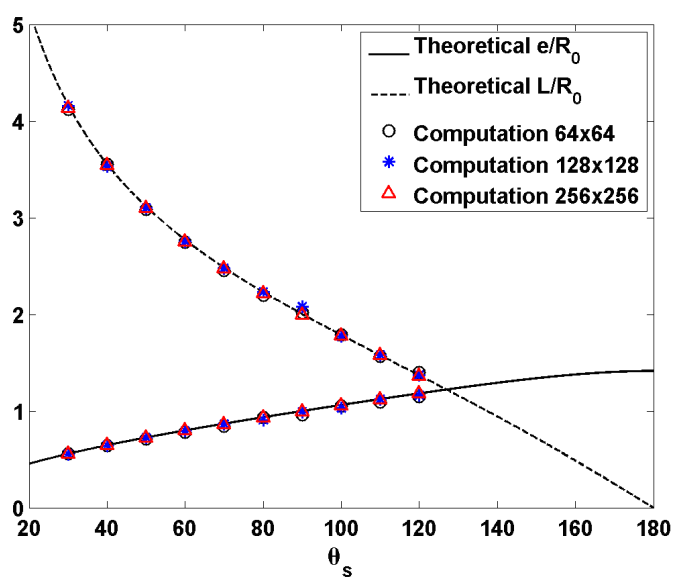

(a) GFCMI

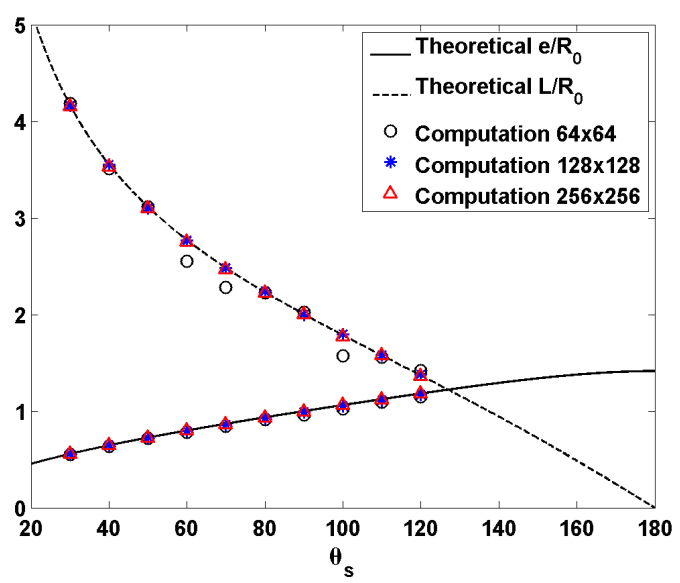

(b) GFSCMI

Figure 18: Results on an inclined wall $\left(\alpha=40^{\circ}\right)$.

equilibrium shape, hence enabling the quantification of spurious currents. Ghost-Fluid methods and more generally sharp-interface methods $[23,48]$ are well known to produce low amplitude spurious currents in comparison to continuum-surface-force methods. However in the framework of a coupling between a two-phase flow solver with an embedded solid boundary, the problem of spurious currents can be much more stringent on the contact line. Consequently, this parameter is of great importance to assess the relevance of such numerical methods. In figure 19, the temporal evolution of the maximum velocity is given, indicating that the spurious currents decrease with the mesh refinement.

The results presented in the table 4 demonstrate that the two methods (GFCSMI and GFCMI) 


\begin{tabular}{|c|c|c|c|c|c|c|c|c|c|}
\hline & & \multicolumn{4}{|c|}{ GFCMI } & \multicolumn{4}{|c|}{ GFSCMI } \\
\hline & & e & $\mathrm{L}$ & $\left\|V_{\max }\right\|$ & $\% \mathrm{Vol}$ & e & $\mathrm{L}$ & $\left\|V_{\max }\right\|$ & $\% \mathrm{Vol}$ \\
\hline \multirow{4}{*}{$\theta=30^{\circ}$} & $64^{2}$ & 0.557 & 4.122 & 0.00224 & 0.975 & 0.553 & 4.181 & 0.0065 & 1 \\
\hline & $128^{2}$ & 0.555 & 4.158 & 0.0015 & 0.998 & 0.559 & 4.16 & 0.015 & 1.002 \\
\hline & $256^{2}$ & 0.556 & 4.136 & 0.00059 & 1 & 0.561 & 4.153 & 0.011 & 1.005 \\
\hline & theoretical & 0.558 & 4.164 & - & - & 0.558 & 4.164 & - & - \\
\hline \multirow{4}{*}{$\theta=60^{\circ}$} & $64^{2}$ & 0.784 & 2.748 & 0.003 & 0.977 & 0.784 & 2.55 & 0.0055 & 0.97 \\
\hline & $128^{2}$ & 0.794 & 2.753 & 0.0071 & 0.990 & 0.794 & 2.766 & 0.0125 & 0.992 \\
\hline & $256^{2}$ & 0.799 & 2.752 & 0.0015 & 0.999 & 0.799 & 2.755 & 0.0121 & 0.995 \\
\hline & theoretical & 0.799 & 2.76 & - & - & 0.799 & 2.76 & - & - \\
\hline \multirow{4}{*}{$\theta=90^{\circ}$} & $64^{2}$ & 0.961 & 2.014 & 0.0083 & 0.962 & 0.965 & 2.026 & 0.009 & 0.97 \\
\hline & $128^{2}$ & 0.992 & 2.08 & 0.0034 & 0.991 & 0.99 & 1.999 & 0.012 & 0.987 \\
\hline & $256^{2}$ & 0.999 & 1.998 & 0.0012 & 1 & 0.997 & 2.002 & 0.007 & 0.995 \\
\hline & theoretical & 1 & 2 & - & - & 1 & 2 & - & - \\
\hline \multirow{4}{*}{$\theta=120^{\circ}$} & $64^{2}$ & 1.151 & 1.397 & 0.012 & 0.975 & 1.147 & 1.418 & 0.028 & 0.976 \\
\hline & $128^{2}$ & 1.164 & 1.369 & 0.0059 & 0.98 & 1.167 & 1.380 & 0.0105 & 0.985 \\
\hline & $256^{2}$ & 1.181 & 1.363 & 0.0061 & 0.996 & 1.185 & 1.363 & 0.0061 & 0.996 \\
\hline & theoretical & 1.18 & 1.365 & - & - & 1.18 & 1.365 & - & - \\
\hline
\end{tabular}

Table 4: Comparison of characteristics of GFCMI and GFSCMI for a slanted wall

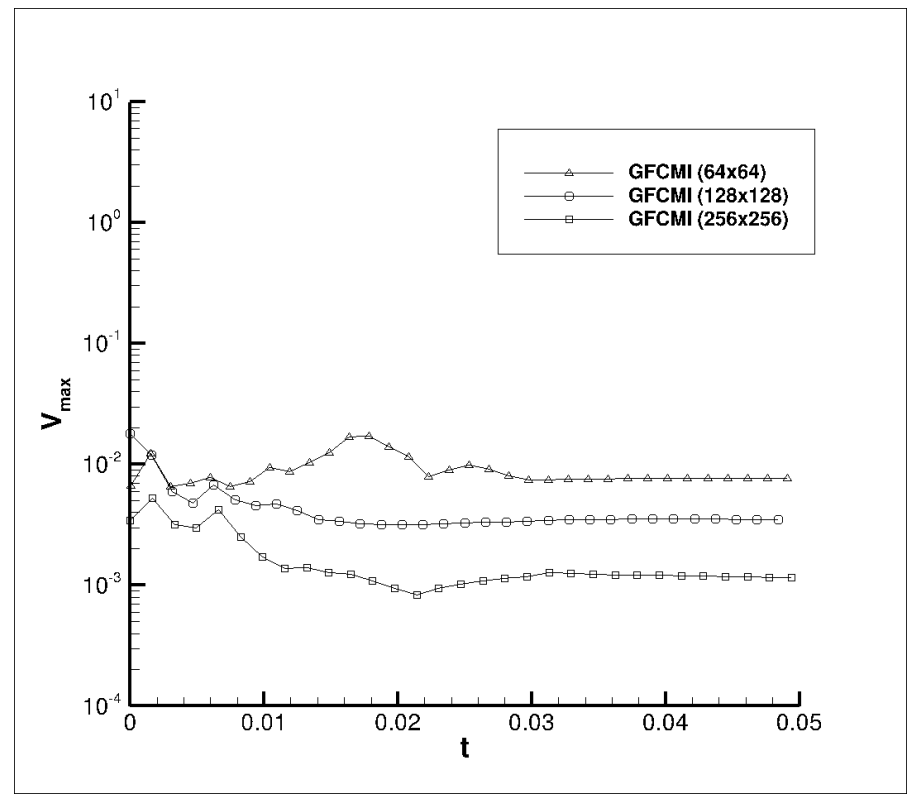

Figure 19: An exemple of the evolution of $\left|V_{\max }\right|$ throughout time on an inclined wall $\left(\alpha=40^{\circ}\right)$ and $\theta_{s}=90^{\circ}$ with GFCMI .

converge toward the expected solution. In some situations, when considering a specific parameter, the errors can increase at one point when the grid is refined. In our opinion, this singular behavior 
should not be alarming since it can be assuredly explained by the compensation of errors acting in an opposite way. This phenomenon occurs frequently when numerical simulations involving complex and strongly coupled mathematical models, see [51] for example. On the one hand GFCMI and GFSCMI provide results of equivalent accuracy if geometrical properties are considered $(e, L$, volume loss). On the other hand GFCMI provides an important gain in accuracy on the amplitude of parasitic currents. Indeed, if the latter is used, the amplitude of the maximum velocity is 5 to 20 times smaller on the finest grid (except if $\theta=120^{\circ}$ where the two methods provide similar results).

\subsection{Two Dimensional Two-Phase Flows in Irregular Domains with Volume Forces}

In this section, the previous study is completed by applying different gravity fields, hence changing the final equilibrium drop's shape. The Bond number $\left(B o=\rho_{L} g R_{0}^{2} / \sigma\right)$ characterizes the gravitational stress applied to the drop, with $B o \in[0,10]$. Results are presented for $\theta_{s}=60^{\circ}$ and $\theta_{s}=120^{\circ}$. Two asymptotic solutions are provided, with one being dominated by capillarity effects and the other by gravity. If the shape of the drop is driven by the surface tension effects, its thickness, $e_{0}$, is deduced from:

$$
e_{0}=R_{0}\left(1-\cos \theta_{s}\right) \sqrt{\frac{\pi}{2\left(\theta_{s}-\sin \theta_{s} \cos \theta_{s}\right)}} .
$$

For $B o>>1$, the drop spreads under the effect of gravity and forms a small puddle with a thickness, $e_{\infty}$, directly proportional to the capillary length:

$$
e_{\infty}=2 \sqrt{\frac{\sigma}{\rho_{L} g}} \sin \left(\frac{\theta_{s}}{2}\right) .
$$

Figure 20 illustrates the shape of a drop at equilibrium on a slanted wall for a static contact angle of $\theta_{s}=60^{\circ}$ and for three different Bond numbers. The simulations are found to be in good agreement with the two asymptotic solutions (23) and (24). One can observe that the transition between these two regimes is approximately for $B o=1$, when gravity and surface tension effects are of the same order of magnitude. GFCMI converges faster, as illustrated in figures 21 and 23. In order to compare more accurately the two methods, details on the simulations are provided in tables 5 and 6 . An illustration of the sharp pressure field is also available in figure 22 .

\begin{tabular}{|c|c||c|c|c||c|c|c|}
\hline \multicolumn{1}{|c||}{} & \multicolumn{3}{c||}{ GFCMI } & \multicolumn{4}{c|}{ GFSCMI } \\
& & $\mathrm{e}$ & $\left\|V_{\text {max }}\right\|$ & $\%$ Vol & $\mathrm{e}$ & $\left\|V_{\text {max }}\right\|$ & $\%$ Vol \\
\hline \multirow{3}{*}{$B o=0.1$} & $64^{2}$ & 0.966 & 0.024 & 0.976 & 0.967 & 0.017 & 0.978 \\
& $128^{2}$ & 0.977 & 0.0082 & 0.991 & 0.979 & 0.0105 & 0.990 \\
& $256^{2}$ & 0.986 & 0.0011 & 0.998 & 0.985 & 0.010 & 0.995 \\
\hline \multirow{3}{*}{$B o=1$} & $64^{2}$ & 0.848 & 0.02 & 0.969 & 0.839 & 0.06 & 0.963 \\
& $128^{2}$ & 0.860 & 0.015 & 0.987 & 0.854 & 0.06 & 0.980 \\
& $256^{2}$ & 0.869 & 0.011 & 0.996 & 0.862 & 0.05 & 0.986 \\
\hline \multirow{3}{*}{$B o=10$} & $64^{2}$ & 0.401 & 0.18 & 0.962 & 0.365 & 0.15 & 0.970 \\
& $128^{2}$ & 0.376 & 0.14 & 0.968 & 0.383 & 0.17 & 0.995 \\
& $256^{2}$ & 0.395 & 0.0102 & 0.982 & 0.390 & 0.2 & 0.952 \\
\hline
\end{tabular}

Table 5: Comparison of characteristics of GFCMI and GFSCMI for a slanted wall $\left(\alpha=40^{\circ}\right)$ and for $\theta_{s}=60^{\circ}$ 


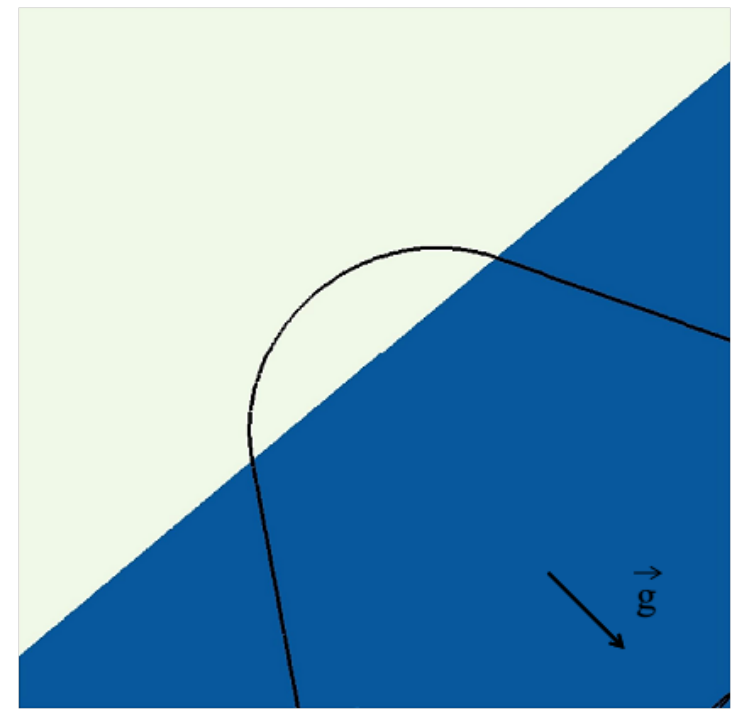

(a) $B o=0.1$

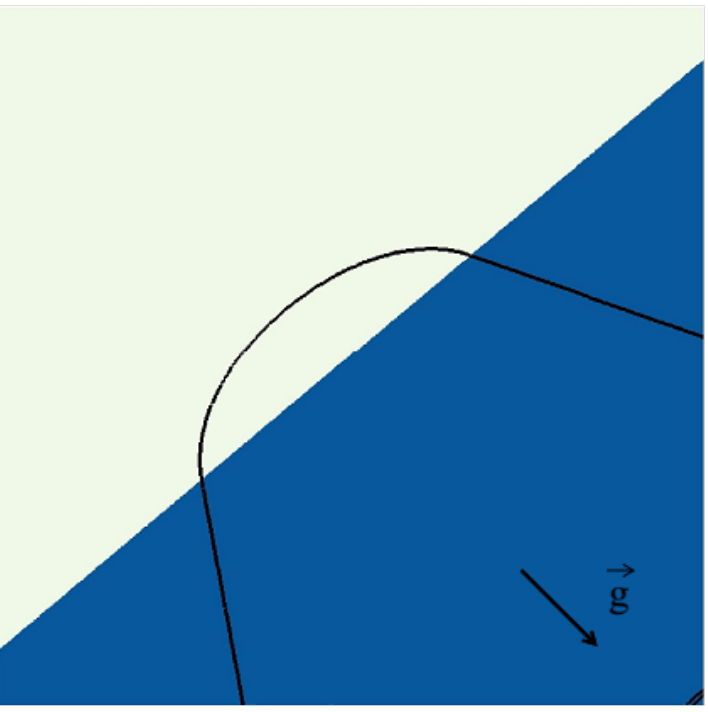

(b) $B o=1$

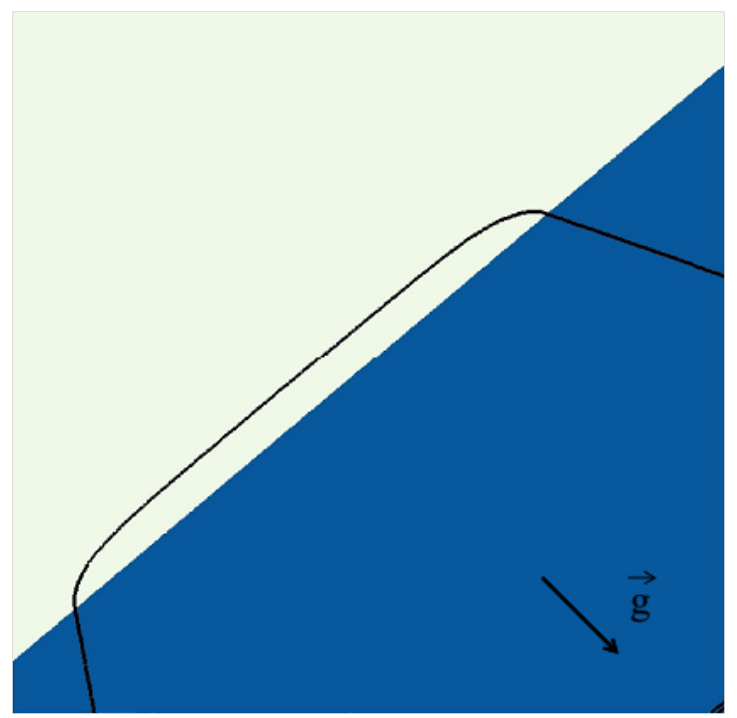

(c) $B o=10$

Figure 20: Illustration of the shape of a drop on a slanted wall for a static contact angle of $\theta_{s}=60^{\circ}$ and for different Bond numbers.

Both methods converge to the same values when the mesh is refined, but as it is the case for the previous example, GFCMI gives better results for lower resolutions. Also, the spurious currents are reduced in the case of GFCMI when the mesh is refined, which is not the case of GFSCMI, hence suggesting a lack of stability of the latter. 


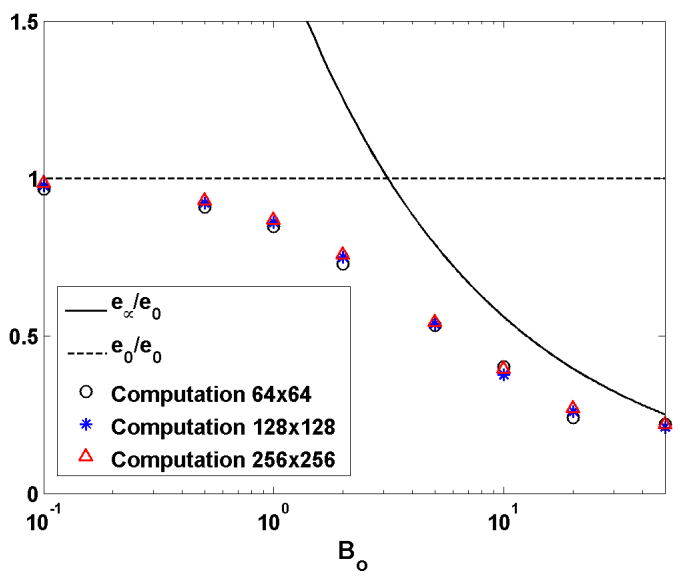

(a) GFCMI

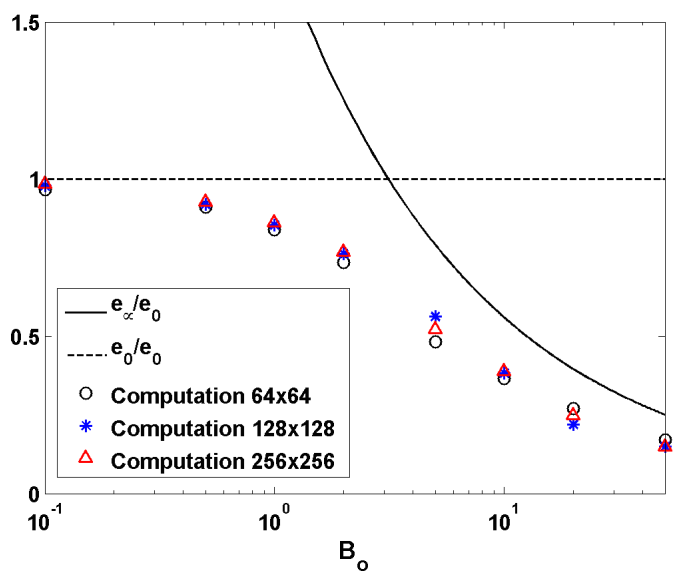

(b) GFSCMI

Figure 21: Results on a slanted wall as a function of $B_{0}$ for $\theta_{s}=60^{\circ}$.
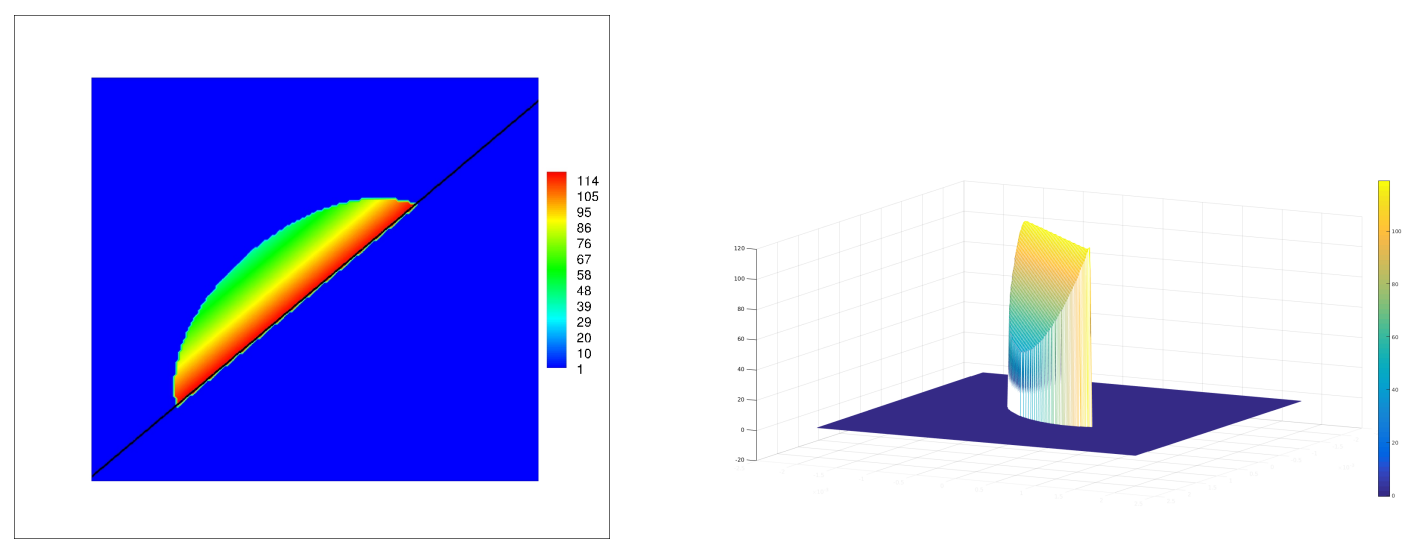

Figure 22: Resulting pressure field $(\mathrm{Pa})$ for $\mathrm{Bo}=1$ and $\theta_{s}=60^{\circ}$.

\subsection{Three Dimensional Two-Phase Flows in Irregular Domains Without Volume Forces}

This study is the same configuration that the one used in section 4.3 except that they represent three dimensional numerical simulations. The slanted wall is chosen in a way as to enforce the wall not to align to the grid in any direction (we take $z=0.85(x+y)$ for $\alpha=40^{\circ}$ ). The contact angle used in this study is $\theta_{s}=120^{\circ}$. The analytical radius, $R$, thickness, $e$ and length, $L$, of the drop are obtained as before, using mass conservation arguments and geometrical properties:

$$
R=R_{0}\left(\frac{2}{\left(2+\cos \theta_{s}\right)\left(1-\cos \theta_{s}\right)^{2}}\right)^{\frac{1}{3}}, \quad L=2 R \sin \theta_{s}, \quad e=R\left(1-\cos \theta_{s}\right) .
$$

Figure 24 illustrates the final shape of the drop on an slanted wall for $\theta_{s}=120^{\circ}$. In table 7 , some comparisons between GFSCMI and GFCMI are presented. Even though the mesh with $64^{3}$ 


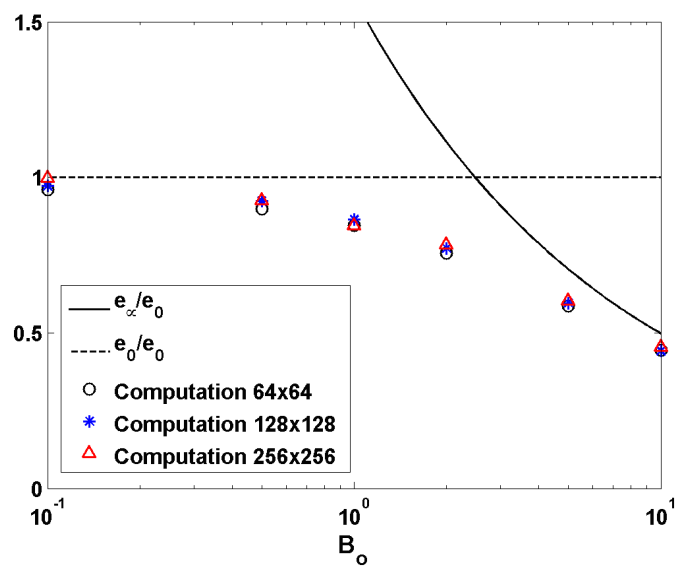

(a) GFCMI

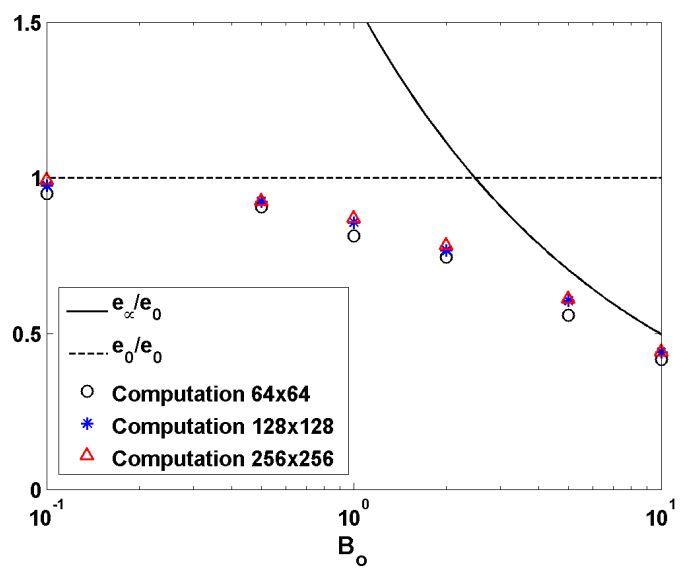

(b) GFSCMI

Figure 23: Results on a slanted wall as a function of $B_{0}$ for $\theta_{s}=120^{\circ}$.

\begin{tabular}{|c|c||c|c|c||c|c|c|}
\hline \multicolumn{1}{|c||}{} & \multicolumn{3}{c||}{ GFCMI } & \multicolumn{4}{c|}{ GFSCMI } \\
& & $\mathrm{e}$ & $\left\|V_{\text {max }}\right\|$ & $\%$ Vol & $\mathrm{e}$ & $\left\|V_{\text {max }}\right\|$ & $\%$ Vol \\
\hline \multirow{3}{*}{$B o=0.1$} & $64^{2}$ & 0.961 & 0.0105 & 0.975 & 0.950 & 0.034 & 0.97 \\
& $128^{2}$ & 0.972 & 0.006 & 0.987 & 0.974 & 0.0105 & 0.992 \\
& $256^{2}$ & 0.998 & 0.0044 & 0.996 & 0.994 & 0.011 & 0.98 \\
\hline \multirow{3}{*}{$B o=1$} & $64^{2}$ & 0.846 & 0.02 & 0.932 & 0.813 & 0.062 & 0.895 \\
& $128^{2}$ & 0.864 & 0.015 & 0.964 & 0.857 & 0.04 & 0.97 \\
& $256^{2}$ & 0.846 & 0.010 & 0.995 & 0.870 & 0.025 & 0.988 \\
\hline \multirow{3}{*}{$B o=10$} & $64^{2}$ & 0.444 & 0.18 & 0.83 & 0.416 & 0.2 & 0.85 \\
& $128^{2}$ & 0.442 & 0.15 & 0.905 & 0.440 & 0.15 & 0.905 \\
& $256^{2}$ & 0.456 & 0.11 & 0.970 & 0.442 & 0.15 & 0.905 \\
\hline
\end{tabular}

Table 6: Comparison of characteristics of GFCMI and GFSCMI for a slanted wall $\left(\alpha=40^{\circ}\right)$ and for $\theta_{s}=120^{\circ}$

\begin{tabular}{|c||cc|}
\hline & $e / R_{0}$ & $L / R_{0}$ \\
\hline \hline Analytical results & 1.26 & 1.45 \\
\hline GFCMI & & \\
$\Delta_{x}=R_{0} / 10$ & 1.16 & 1.47 \\
$\Delta_{x}=R_{0} / 20$ & 1.22 & 1.45 \\
\hline GFSCMI & & \\
$\Delta_{x}=R_{0} / 10$ & 1.17 & 1.48 \\
$\Delta_{x}=R_{0} / 20$ & 1.20 & 1.46 \\
\hline
\end{tabular}

Table 7: Geometrical characteristics of the final shape of the drop on a slanted wall $\left(\alpha=40^{\circ}\right)$ in $3 \mathrm{D}$ for $\theta_{s}=120^{\circ}$.

cells is not fine enough to model the behavior of the drop, the error is still less than $9 \%$ for the analytical values of the thickness of the drop between the two methods. The error is reduced when the mesh is $128^{3}$, with an error of $3.1 \%$ for GFCMI and $4.7 \%$ for GFSCMI. 


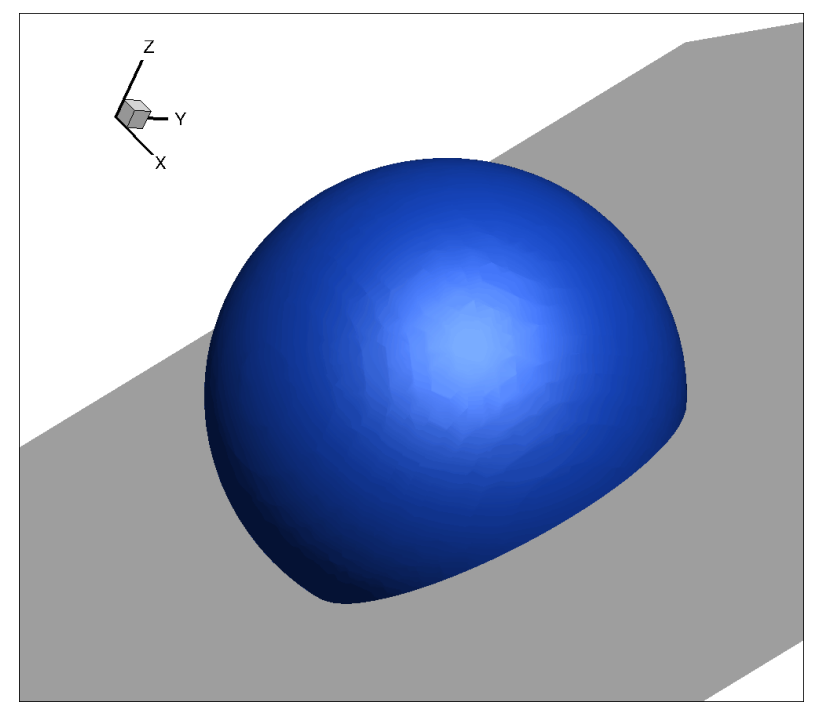

Figure 24: Final shape of the drop on an slanted wall for $\theta_{s}=120^{\circ}$.

\subsection{Half-Filled Rotating Spherical Tank}

In order to highlight the applicability of the numerical methods presented in this paper, we now describe the benchmark of a rotating spherical tank half-filled with water, with the other half filled with a gas. The center of the spherical tank is located at a distance $x=L$, from a rotational axis along the $z$-axis. Consequently, the spherical tank is rotating in the $x y$-plane. As our simulations will be performed in the tank reference frame $R^{\prime}$, it is necessary to account for the inertial forces, which can be decomposed into two terms in the Navier-Stokes equations:

$$
\mathbf{F}_{\mathbf{v}}=-\rho \mathbf{a}_{1}-\rho \mathbf{a}_{2},
$$

with

$$
\mathbf{a}_{1}=\boldsymbol{\Omega}_{R^{\prime} / R} \times\left(\boldsymbol{\Omega}_{R^{\prime} / R} \times \mathbf{O M}\right) \quad \text { and } \quad \mathbf{a}_{2}=\mathbf{2} \boldsymbol{\Omega}_{R^{\prime} / R} \times \mathbf{V}_{R^{\prime}}(M),
$$

where $\mathbf{a}_{1}$ represents the centrifugal acceleration and $\mathbf{a}_{2}$ denotes the Coriolis acceleration. We also denote by $\boldsymbol{\Omega}_{R^{\prime} / R}=\omega \mathbf{e}_{\mathbf{z}}$ the rotation vector and $\mathbf{O M}=(L+x) \mathbf{e}_{\mathbf{x}}+y \mathbf{e}_{\mathbf{y}}+z \mathbf{e}_{\mathbf{z}}$ the position vector of a fluid particle located at $M$ in the tank's reference frame. Since the gravity force acts along the $z$-axis, we obtain the following expression for the three components of the volume force in the Navier-Stokes equations:

$$
F_{x}=\rho\left(-2 \omega v+\omega^{2}(L+x)\right), \quad F_{y}=\rho\left(2 \omega u+\omega^{2} y\right), \quad \text { and } \quad F_{z}=-\rho g_{z} .
$$

In this simulation example, we are interested in computing the forces acting on the wall of the tank due to the fluid motion. In the simple configuration, which is presented in this section, a steady theoretical solution of the interface shape and of the pressure force can be obtained. Considering the characteristic length of the tank, this benchmark emphasizes the volume forces effects over the capillary effects on the resulting flow. The equilibrium shape of the interface is resulting from the enforced contact angle and the volume forces balance. This enables us to assess the accuracy of our numerical methods in a situation where the fluid motion induced by non-inertial forces is coupled to 
a three-dimensional simulation in an irregular geometry. We have imposed a contact angle between the liquid-gas interface and the solid tank boundary equal to $\theta_{s}=90^{\circ}$.

In the general case, the liquid-gas interface will evolve towards a parabolic-like shape. However, it can be demonstrated that in our configuration, if $R / L \ll 1$, the liquid-gas interface will be a flat surface forming an angle equal to $\theta_{e q}=\tan ^{-1}\left(\frac{\omega^{2} L}{g_{z}}\right)$ with the $x y$-plane.

The theoretical expression of the resulting force is obtained from the integration of the pressure field on the surface of the tank. Let's start with the equilibrium of the forces :

$$
\nabla p=-\rho \mathbf{a}_{\mathbf{e}}+\rho \mathbf{g} \quad \text { with } \quad \mathbf{a}_{\mathbf{e}}=-\omega^{2}\left((L+x) \mathbf{e}_{\mathbf{x}}+y \mathbf{e}_{\mathbf{y}}\right) .
$$

Therefore the decomposition of the pressure gradient in each direction is :

$$
\frac{\partial p}{\partial x}=\rho \omega^{2}(L+x), \quad \frac{\partial p}{\partial y}=\rho \omega^{2} y \quad \text { and } \quad \frac{\partial p}{\partial z}=-\rho g_{z} .
$$

Thus with an integration of the pressure field one obtains :

$$
p(x, y, z)=\rho \omega^{2}\left(L x+\frac{x^{2}+y^{2}}{2}\right)-\rho g_{z} z+p_{0} .
$$

In order to compute the force, one must integrate this pressure on the wall of the spherical tank. The integration per direction gives :

$$
P_{x}=\frac{4 \pi}{3} \rho \omega^{2} L R^{3}, \quad P_{y}=0, \quad P_{z}=-\frac{4 \pi}{3} \rho g_{z} R^{3} .
$$

The resulting force is expressed here with $\rho_{l}$ and divided by 2 , as the tank is half filled with water :

$$
\mathbf{F}_{\mathbf{v}_{\mathbf{s} \rightarrow \mathbf{f}}}=\frac{2}{3} \pi R^{3} \rho_{l}\left(\omega^{2} L \mathbf{e}_{\mathbf{x}}-g_{z} \mathbf{e}_{\mathbf{z}}\right) .
$$

The fluid properties are close to that of water for the liquid and that of air for the gas, i.e. we take $\rho_{L}=1000 \mathrm{~kg} \cdot \mathrm{m}^{-3}, \mu_{L}=10^{-3} \mathrm{~kg} \cdot \mathrm{m}^{-1} \cdot \mathrm{s}^{-1}, \rho_{G}=1 \mathrm{~kg} \cdot \mathrm{m}^{-3}, \mu_{G}=10^{-5} \mathrm{~kg} \cdot \mathrm{m}^{-1} \cdot \mathrm{s}^{-1}$ and $\sigma=7 \times 10^{-2} \mathrm{~N} \cdot \mathrm{m}^{-1}$. The radius of the spherical tank is $R=2.5 \times 10^{-2} \mathrm{~m}$ and the acceleration due to gravity is equal to $g_{z}=9.81 \mathrm{~m} \cdot \mathrm{s}^{-2}$. We have performed simulations for the four configurations given in table 8 .

\begin{tabular}{|c||c|c|c|c|}
\hline & Case $A$ & Case $B$ & Case $C$ & Case $D$ \\
\hline$\frac{\omega^{2} L}{g_{z}}$ & 0.176 & 1 & 5.67 & 1 \\
\hline$\frac{R}{L}$ & 0.05 & 0.05 & 0.05 & 1 \\
\hline
\end{tabular}

Table 8: Configuration of the four cases 


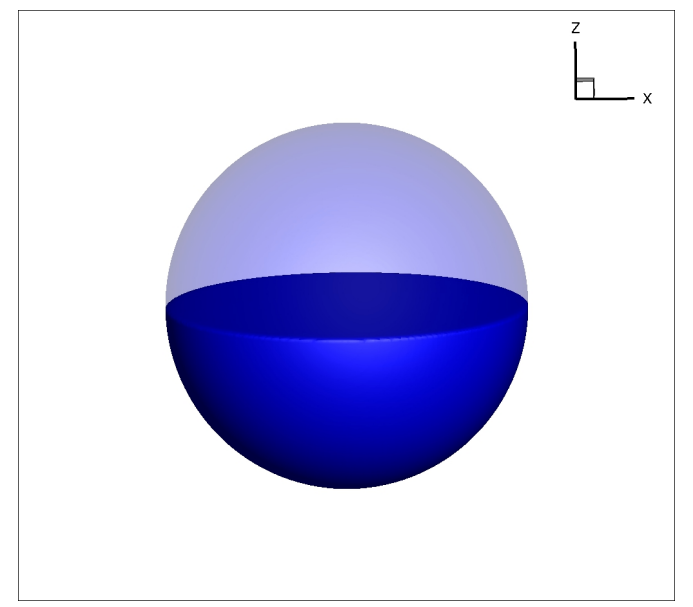

Figure 25: Initial position of the interface for the four simulations of the rotating spherical tank.

For large time, a flat interface is expected in cases $A, B$ and $C$ with $\theta_{e q}=10^{\circ}, 45^{\circ}$ and $80^{\circ}$. For case $D$, considering that the ratio $R / L$ is not negligible anymore, a parabolic-like shape of the interface is observed. This can be demonstrated by the former equation depicting the pressure field as :

$$
p(x, y, z)=\rho \omega^{2}\left(L x+\frac{x^{2}+y^{2}}{2}\right)-\rho g_{z} z+p_{0} .
$$

Indeed the interface is an isobar $p(x, y, z)=p_{0}$, so the shape of the resulting interface in the following plan equation would be :

$$
z=f(x, y)=\frac{\omega^{2}}{g_{z}}\left(L x+\frac{x^{2}+y^{2}}{2}\right) .
$$

If $L>>R$ then the linear term $L x$ of the equation will dominate the flow, giving a plan interface, but when $L \simeq R$ then the quadratic term is not negligible anymore and gives the shape of a parabola to the interface.

\begin{tabular}{|c||c|c||c|c||c|c|}
\hline \multicolumn{1}{|c||}{} & \multicolumn{2}{c||}{ Case A } & \multicolumn{2}{c|}{ Case B } & \multicolumn{2}{c|}{ Case C } \\
\hline & $F_{x}(N)$ & $F_{z}(N)$ & $F_{x}(N)$ & $F_{z}(N)$ & $F_{x}(N)$ & $F_{z}(N)$ \\
\hline $64^{3}$ & 0.05574 & -0.3166 & 0.3189 & -0.3518 & 1.746 & -0.302 \\
\hline $128^{3}$ & 0.05618 & -0.3234 & 0.3358 & -0.3330 & 1.974 & -0.337 \\
\hline Theory & 0.0566 & -0.321 & 0.321 & -0.321 & 1.82 & -0.32 \\
\hline
\end{tabular}

Table 9: Comparisons between theoretical and the computational results (for two different grids) of the pressure force acting on the wall for cases $A, B$, and $C$.

The initial interface (in blue), depicted in figure 26, is simply a horizontal plane in all four cases. The boundary of the spherical tank and the axis orientation of the computational field is also depicted in figure 26. All the simulations are carried out until the final time $t=5 \mathrm{~s}$. In figure 26 , the final steady shape of the interface obtained with a $128^{3}$ grid is plotted for the four cases. 

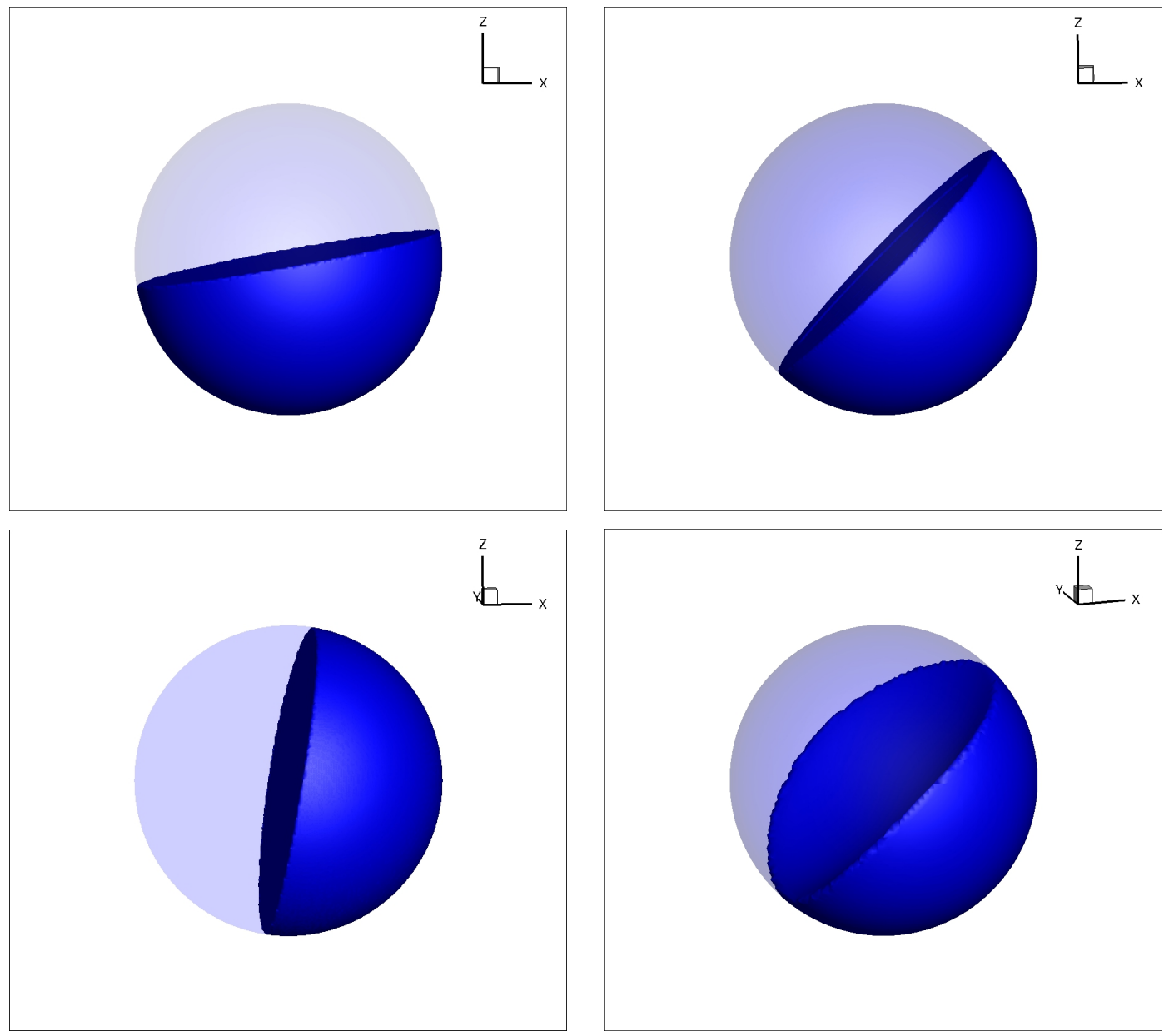

Figure 26: Final steady position of the interface for the four simulations (cases A, B, C and D) of the rotating spherical tank (grid $\left.128^{3}\right)$.

As expected, a flat sloped interface is observed in the simulations of cases $A, B$ and $C$. In case $D$, we clearly observe a parabolic-like shaped interface, in agreement with theory. Quantitative comparisons between theoretical and computational results are presented in table 9 . In figure 27 , the interface location and the pressure field are plotted in the $x z$-plane for case $B$ and $D$, where we used a grid with size $128^{3}$. This gives a more accurate visualization of the interface shape and of the pressure isocontours.

\section{Conclusion}

In this paper, several numerical methods are proposed to compute numerical simulations of liquid-gas flows with an embedded solid boundary inside a Cartesian grid. Our numerical method is presented as an extension of the previous work in [34], on a coupling between a one-phase flow and 

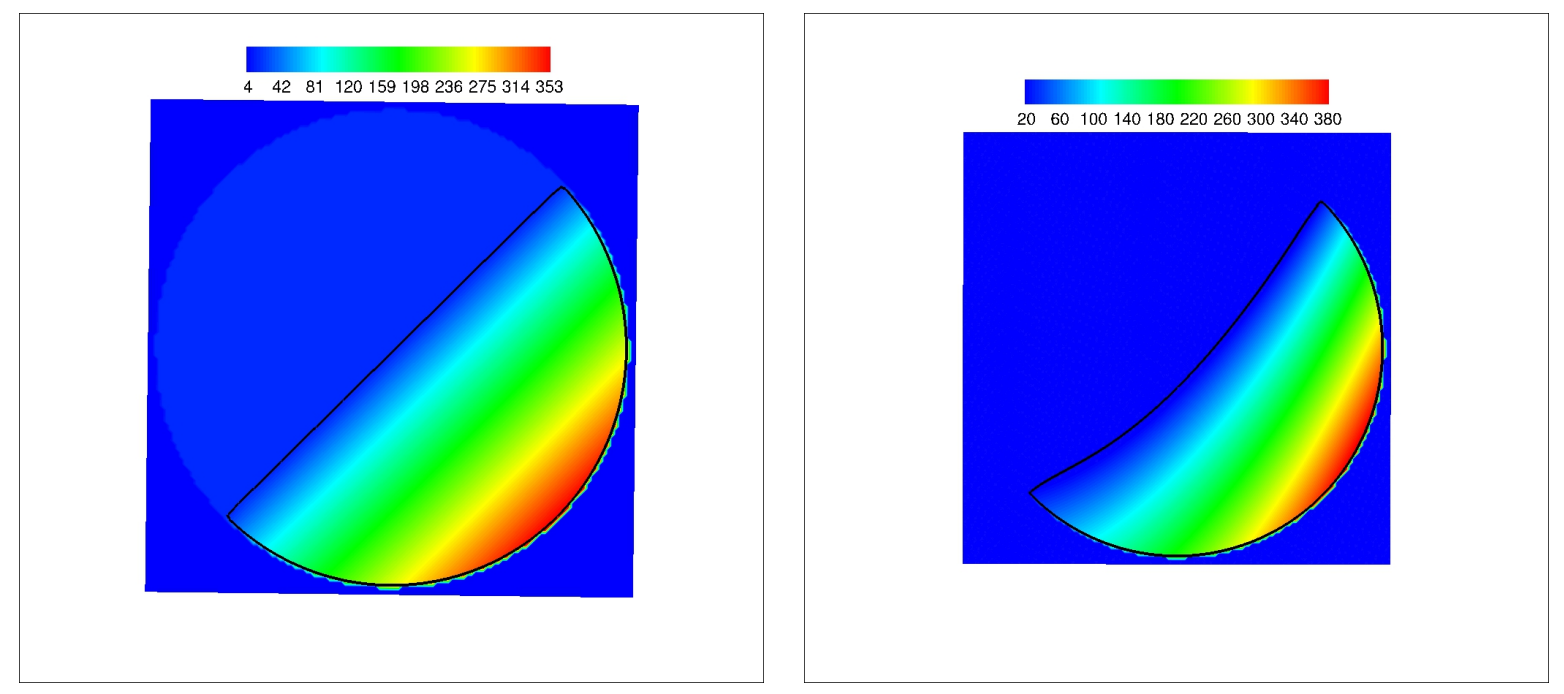

Figure 27: Final steady position of the interface and pressure field (Pa) for the four simulations (cases $A, B, C$ and $D$ ) of the rotating spherical tank for cases $B$ and $D\left(\right.$ grid $\left.128^{3}\right)$.

a solid, to two-phase flows simulations where contact lines can be formed at the boundary between the three phases. Several challenges must be faced to compute accurately the surface tension effects, the viscous effects, the contact angle and the volume forces in this more complex framework. We propose a detailed analysis and suitable solutions to overcome these difficulties. Next, accurate benchmarks are defined and numerical simulations are performed in order to assess the suitable behavior of our numerical method in various situations. As an implicit temporal discretization is required to compute the viscous terms in the grid cells which are crossed by the solid-fluid interface, we compare two possible implementations of this implicit temporal discretization. This comparison brings out that the Conservative formulation (GFCMI) of the viscous terms should be favored instead of the Semi-Conservative formulation (GFSCMI), since an important decrease of the spurious currents on the contact line is observed with the conservative one.

\section{Acknowledgements}

Mathieu Lepilliez and Sébastien Tanguy gratefully acknowledge funding by Airbus Defence \& Space and the CNES (the French national space agency) in the context of the "sloshing in satellite tank under micro-gravity influence" program. Frederic Gibou gratefully acknowledges funding from ONR N00014-11-1-0027. 


\section{Appendix A. Detailed derivation of the splitting of the viscous-stress operator}

In this appendix, we give some more insight on the splitting of the viscous-stress tensor. In section 2, equation 2 states:

$$
\nabla \cdot(2 \mu \underline{\underline{\mathbf{D}}})=2 \mu \nabla \cdot(\underline{\underline{\mathbf{D}}})+2[\mu] \frac{\partial u_{n}}{\partial n} \delta_{\Gamma} \mathbf{n}
$$

This demonstration is given in $2 \mathrm{D}$ for the reader but can be extended to $3 \mathrm{D}$ without any difficulties. Let us explicitly write the equation on the normal basis, such as :

$$
\begin{aligned}
& \nabla \cdot(2 \mu \underline{\underline{\mathbf{D}}})=\left(\begin{array}{ll}
\frac{\partial}{\partial n} & \frac{\partial}{\partial t}
\end{array}\right) \cdot\left(\begin{array}{cc}
2 \mu \frac{\partial u_{n}}{\partial n} & \mu\left(\frac{\partial u_{t}}{\partial n}+\frac{\partial u_{n}}{\partial t}\right) \\
\mu\left(\frac{\partial u_{t}}{\partial n}+\frac{\partial u_{n}}{\partial t}\right) & 2 \mu \frac{\partial u_{t}}{\partial t}
\end{array}\right) \\
& =\frac{\partial}{\partial n}\left(2 \mu \frac{\partial u_{n}}{\partial n}\right)+\mu\left(\frac{\partial^{2} u_{t}}{\partial t \partial n}+\frac{\partial^{2} u_{n}}{\partial t^{2}}\right)+\frac{\partial}{\partial n}\left(\mu\left(\frac{\partial u_{t}}{\partial n}+\frac{\partial u_{n}}{\partial t}\right)\right)+2 \mu \frac{\partial^{2} u_{t}}{\partial t^{2}} .
\end{aligned}
$$

The tangential derivatives are straightforward as no particular effects appear in this direction (no jump conditions) and the viscosity is piecewise constant. This is less natural for the normal derivatives, as jump conditions appear at the interface. In [27], the authors demonstrate that $\frac{\partial}{\partial n}\left(2 \mu \frac{\partial u_{n}}{\partial n}\right)$ is continuous throughout the interface, and can therefore be derived as :

$$
\frac{\partial}{\partial n}\left(2 \mu \frac{\partial u_{n}}{\partial n}\right)=2[\mu] \frac{\partial u_{n}}{\partial n} \delta_{\Gamma} \mathbf{n}+2 \mu \frac{\partial^{2} u_{n}}{\partial n^{2}} .
$$

For the cross-term derivative we refer to [6], where Brackbill demonstrates that $\mu\left(\frac{\partial u_{t}}{\partial n}+\frac{\partial u_{n}}{\partial t}\right)=$ $\frac{\partial \sigma}{\partial s}$, where $\sigma$ is the surface tension and $s$ the curvilinear coordinate along the interface. Therefore if no Marengoni effects appear, the derivative is continuous throughout the interface, and can also be computed as :

$$
\frac{\partial}{\partial n}\left(\mu\left(\frac{\partial u_{t}}{\partial n}+\frac{\partial u_{n}}{\partial t}\right)\right)=\mu\left(\frac{\partial^{2} u_{t}}{\partial n^{2}}+\frac{\partial^{2} u_{n}}{\partial n \partial t}\right)
$$

as there are no additional singular source term on the interface, and the viscosity is piecewise constant. Finally, we can resume this demonstration :

$$
\begin{aligned}
\nabla \cdot(2 \mu \underline{\underline{\mathbf{D}}}) & =\frac{\partial}{\partial n}\left(2 \mu \frac{\partial u_{n}}{\partial n}\right)+\mu\left(\frac{\partial^{2} u_{t}}{\partial t \partial n}+\frac{\partial^{2} u_{n}}{\partial t^{2}}\right)+\frac{\partial}{\partial n}\left(\mu\left(\frac{\partial u_{t}}{\partial n}+\frac{\partial u_{n}}{\partial t}\right)\right)+2 \mu \frac{\partial^{2} u_{t}}{\partial t^{2}} \\
& =2[\mu] \frac{\partial u_{n}}{\partial n} \delta_{\Gamma} \mathbf{n}+2 \mu \frac{\partial^{2} u_{n}}{\partial n^{2}}+\mu\left(\frac{\partial^{2} u_{t}}{\partial t \partial n}+\frac{\partial^{2} u_{n}}{\partial t^{2}}\right)+\mu\left(\frac{\partial^{2} u_{t}}{\partial n^{2}}+\frac{\partial^{2} u_{n}}{\partial n \partial t}\right)+2 \mu \frac{\partial^{2} u_{t}}{\partial t^{2}} \\
& =2 \mu \nabla \cdot(\underline{\underline{\mathbf{D}}})+2[\mu] \frac{\partial u_{n}}{\partial n} \delta_{\Gamma} \mathbf{n} .
\end{aligned}
$$

\section{Appendix B. Some remarks on the temporal discretization of the viscous terms}

As observed in section 3.3, the resulting matrix from the temporal discretization of the viscous terms is diagonally dominant depending on the time step constraint. Indeed, this hypothesis is verified if : 


$$
\rho>>\frac{\mu}{\Delta x^{2}} \Delta t
$$

with $\Delta t \simeq \Delta t_{\text {surf_tens }}=\sqrt{\frac{\rho}{\sigma} \Delta x^{3}}$, as the capillary time step is usually the smaller time step encountered in multiphase flow configurations (and if an implicit temporal discretization of the viscous term is used). Therefore,

$$
\rho>>\frac{\mu}{\Delta x^{2}} \sqrt{\frac{\rho}{\sigma} \Delta x^{3}}
$$

Hence, the condition to obtain a diagonally dominant system is :

$$
1>>\sqrt{\frac{\mu^{2}}{\rho^{2} \Delta x^{4}} \frac{\rho}{\sigma} \Delta x^{3}}=\frac{\mu}{\sqrt{\Delta x \sigma \rho}}=O h_{\Delta x}
$$

with $O h_{\Delta x}$ the Ohnesorge number based on the cell size of the mesh. Let us remark that in our applications, this number respects this condition for typical fluids such as water and air flows, with $\Delta x \in\left[10^{-6} ; 10^{-4}\right] m, O h_{\Delta x} \in\left[10^{-2} ; 10^{-1}\right]$.

\section{Appendix C. Rising spherical bubbles at low Reynolds numbers}

In this appendix, a study is carried out to compare the different projection methods on regular domains. This study was initially proposed in [25] and is completed in this paper with GFCMI.

A spherical air bubble is placed in water under gravity stress. This study is adequate to compare how the different projection methods affect the viscous terms as no drag is generated by the pressure in this flow. These are 2D axisymmetric numerical simulations with a variation of the Reynolds number, and two different Bond numbers, in order to keep the spherical shape. The characteristics of the numerical simulations are reported in table C.10.

\begin{tabular}{|c|c|c|c|c|c|}
\hline & $R e_{\infty}=20$ & $R e_{\infty}=40$ & $R e_{\infty}=60$ & $R e_{\infty}=80$ & $R e_{\infty}=100$ \\
\hline$R_{\text {bubble }}[\mu \mathrm{m}]$ & 19.55 & 44.65 & 70.45 & 98.95 & 127.9 \\
\hline$g_{z}\left[\mathrm{~m} \cdot \mathrm{s}^{-2}\right]$ & -9144.4 & -1755.2 & -705.6 & -357.5 & -213.9 \\
\hline$\sigma\left[\mathrm{N} \cdot \mathrm{m}^{-1}\right]$ & 0.56 & 0.56 & 0.56 & 1.12 & 1.12 \\
\hline$t_{f}[\mathrm{~s}]$ & 0.0004 & 0.00135 & 0.002375 & 0.0048 & 0.007 \\
\hline
\end{tabular}

Table C.10: Parameters used in the numerical experiment of this appendix.

The numerical domain is chosen in order not to disturb the rise of the bubble, with $L_{r}=$ $8 R_{\text {bubble }}$ and $L_{z}=4 L_{r}$. The physical properties of the two fluids are those of air and water, i.e., $\rho_{L}=1000 \mathrm{~kg} \cdot \mathrm{m}^{-3}, \mu_{l i q}=0.00113 \mathrm{~kg} \cdot \mathrm{m}^{-1} \cdot \mathrm{s}^{-1}, \rho_{\text {gas }}=1.226 \mathrm{~kg} \cdot \mathrm{m}^{-3}, \mu_{\text {gas }}=1.78 \mathrm{~kg} \cdot \mathrm{m}^{-1} \cdot \mathrm{s}^{-1}$. Table C.10 gives the other physical parameters for five different configurations corresponding to $R e=20,40$ and 60 for the Bond number $B o=0.025$ and to $R e=80,100$ for $B o=0.0125$. Figure C.28 illustrates the results for the simulation of a rising air bubble where the final Reynolds number is $R e=60$. The numerical simulations are conducted for three different meshes, $32 \times 128,64 \times 256$ and $128 \times 512$ corresponding respectively to 8,16 and 32 cells per diameter.

Results obtained with GFCMI are presented in table C.11. For completeness, we also include the results presented in [25], namely the results obtained for GFCM (table C.12), in which the 


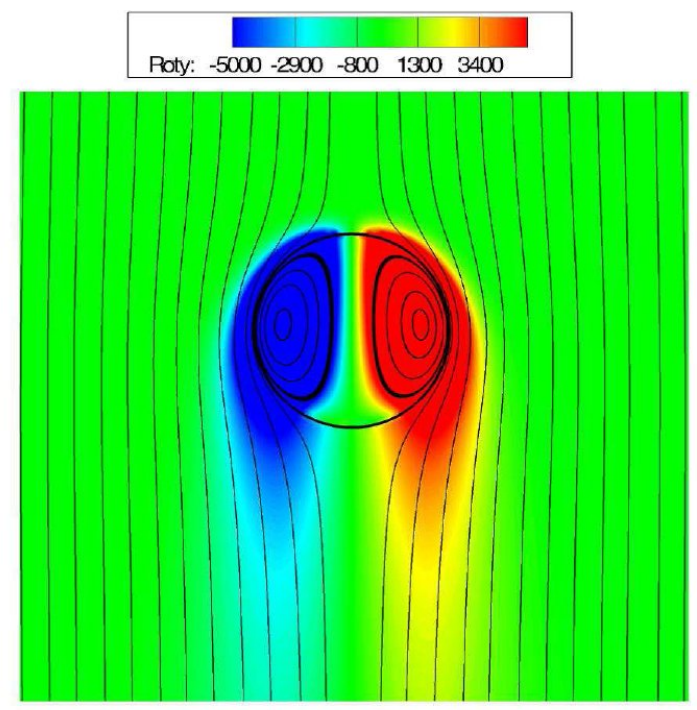

Figure C.28: Streamlines and vorticity contours $\left(s^{-1}\right)$ in the moving referential of the rising bubble for $R e=60$.

\begin{tabular}{|c|c|c|c|c|c|}
\hline Mesh & $R e_{\infty}=20$ & $R e_{\infty}=40$ & $R e_{\infty}=60$ & $R e_{\infty}=80$ & $R e_{\infty}=100$ \\
\hline $32 \times 128$ & $-22.4 \%$ & $-32.2 \%$ & $-37.2 \%$ & $-75.53 \%$ & $-43.35 \%$ \\
\hline $64 \times 256$ & $+2.44 \%$ & $+3.12 \%$ & $+1.07 \%$ & $-1.78 \%$ & $-1.30 \%$ \\
\hline $128 \times 512$ & $+2.38 \%$ & $+2.38 \%$ & $+0.37 \%$ & $+0.8 \%$ & $+0.19 \%$ \\
\hline
\end{tabular}

Table C.11: Error on the final Reynolds number value for rising air bubbles with GFCMI.

\begin{tabular}{|c|c|c|c|c|c|}
\hline Mesh & $R e_{\infty}=20$ & $R e_{\infty}=40$ & $R e_{\infty}=60$ & $R e_{\infty}=80$ & $R e_{\infty}=100$ \\
\hline $32 \times 128$ & $-22.0 \%$ & $-34.0 \%$ & $-38.8 \%$ & $-63.0 \%$ & $-67.4 \%$ \\
\hline $64 \times 256$ & $+2.5 \%$ & $+1.8 \%$ & $-1.3 \%$ & $-1.3 \%$ & $-3.0 \%$ \\
\hline $128 \times 512$ & $+0.5 \%$ & $+0.8 \%$ & $-1.0 \%$ & $+1.9 \%$ & $+1.9 \%$ \\
\hline
\end{tabular}

Table C.12: Error on the final Reynolds number value for rising air bubbles with the GFCM.

\begin{tabular}{|c|c|c|c|c|c|}
\hline Mesh & $R e_{\infty}=20$ & $R e_{\infty}=40$ & $R e_{\infty}=60$ & $R e_{\infty}=80$ & $R e_{\infty}=100$ \\
\hline $32 \times 128$ & $-18.6 \%$ & $-25.0 \%$ & $-30.2 \%$ & $-52.9 \%$ & $-55.1 \%$ \\
\hline $64 \times 256$ & $-1.2 \%$ & $+1.5 \%$ & $-0.1 \%$ & $+2.0 \%$ & $+0.9 \%$ \\
\hline $128 \times 512$ & $-3.7 \%$ & $+0.1 \%$ & $-0.2 \%$ & $+3.2 \%$ & $+3.9 \%$ \\
\hline
\end{tabular}

Table C.13: Error on the final Reynolds number value for rising air bubbles with the GFSCMI.

viscous terms were computed using an explicit scheme, the results obtained for GFSCMI (table C.13), and those obtained for GFSCM (table C.14), where the viscous terms were computed with an explicit scheme.

First of all, one can observe that the mesh $32 \times 128$ cannot describe the viscous effects accurately, as the viscous boundary layer is not thick enough to be fully resolved. For the other meshes, the 


\begin{tabular}{|c|c|c|c|c|c|}
\hline Mesh & $R e_{\infty}=20$ & $R e_{\infty}=40$ & $R e_{\infty}=60$ & $R e_{\infty}=80$ & $R e_{\infty}=100$ \\
\hline $32 \times 128$ & $-23.5 \%$ & $-32.5 \%$ & $-9.0 \%$ & $-62.5 \%$ & $-67.0 \%$ \\
\hline $64 \times 256$ & $-0.5 \%$ & $+2.5 \%$ & $+1.3 \%$ & $+4.3 \%$ & $+3.0 \%$ \\
\hline $128 \times 512$ & $-3.5 \%$ & $+0.6 \%$ & $+0.7 \%$ & $+5.4 \%$ & $+6 \%$ \\
\hline
\end{tabular}

Table C.14: Error on the final Reynolds number value for rising air bubbles with the GFSCM with an explicit scheme used to compute the viscous terms.

results are satisfactory with an error under $3 \%$ in most cases.

The results obtained with GFCM and GFCMI indicate convergence with less than $3 \%$ on every cases. GFSCM and GFSCMI do not give results that are as accurate. This study thus indicates that GFCM and GFCMI are better suited for modeling viscous effects in multiphase flows, and confirms that GFCMI is a reliable method to compute the viscous terms in the case of irregular domains. 


\section{References}

[1] S. Afkhami, S. Zaleski, M. Bussmann, A mesh-dependent model for applying dynamic contact angles to VOF simulations, J. Comput. Phys. 228 (2009) 5370-5389.

[2] A.S. Almgren, J.B. Bell, P. Colella, L. H. Howell, M. L. Welcome, A Conservative Adaptive Projection Method for the Variable Density Incompressible NavierStokes Equations, J. Comput. Phys. 1 (1998) 1-46.

[3] T. Aslam, A partial differential equation approach to multidimensional extrapolation, J. Comput. Phys. 193 (2004) 349-355.

[4] P. A. Berthelsen and O. M. Faltinsen. A local directional ghost cell approach for incompressible viscous flow problems with irregular boundaries, J. Comput. Phys 227 (2008) 4354-4397.

[5] R. Borges, M. Carmona, B. Costa, W.S. Don, An improved weighted essentially non-oscillatory scheme for hyperbolic conservation laws, J. Comput. Phys. 227 (2008) 3191-3211.

[6] J.U. Brackbill, D.B. Kothe, C. Zemach, A continuum Method for Modeling Surface Tension, J. Comput. Phys. 100 (1992) 335-354.

[7] M. Braza, P. Chassaing, H. Ha Minh, Numerical study and physical analysis of the pressure and velocity fields in the near wake of a circular cylinder, Journal of Fluids Mechanics 165 (1986) 79-130.

[8] B. Bunner, G. Tryggvason ,Dynamics of homogeneous bubbly flows Part 1. Rise velocity and microstructure of the bubbles, J. Fluid Mech., 446 (2002) 17-52.

[9] D. Calhoun, A cartesian grid method for solving the two-dimensional streamfunction-vorticity equations in irregular regions, J. Comput. Phys. 176 (2002) 231-275.

[10] J. Choi, R.C. Oberoi, J. R. Edwards, and J. A. Rosati, An immersed boundary method for complex incompressible flows, J. Comput. Phys. 224 (2007) 757-784.

[11] A. Chorin, A numerical method for solving incompressible viscous flow problems, J. Comput. Phys. 2 (1967) 12-26.

[12] R. Coutanceau, M. et Bouard. Experimental determination of main features of the viscous flow in the wake of a circular cylinder in uniform translation. part 1 steady flow. Journal of Fluids Mechanics, 79 (1977) 4018-4037.

[13] J.E. Dendy, Black box multigrid, J. Comput. Phys. 48 (3) (1982) 366386.

[14] J-B. Dupont and D. Legendre. Numerical simulation of static and sliding drop with contact angle hysteresis, J. Comput. Phys. 229(2010) 2453-2478.

[15] R. Fedkiw, T. Aslam, B. Merriman, S. Osher, A non-oscillatory Eulerian approach to interfaces in multimaterial flows (The Ghost-Fluid Method), J. Comput. Phys. 152 (1999) 457-492.

[16] M. Gazzola, P. Chatelain, W.M. Van Rees, P. Koumoutsakos, Simulations of single and multiple swimmers with non-divergence free deforming geometries, J. Comput. Phys. 230 (2011) 70937114 . 
[17] P.G de Gennes, Wetting: statics and dynamics, Rev. Mod. Phys. 57 (1985) 827.

[18] F. Gibou, R. Fedkiw, L.-T. Cheng, and M. Kang. A second-order-accurate symmetric discretization of the poisson equation on irregular domains, J. Comput. Phys. 176 (2002) 205-227.

[19] F. Gibou, L. Chen, D. Nguyen, S. Banerjee, A level-set based sharp interface method for the multiphase incompressible Navier-Stokes equations with phase change, J. Comput. Phys. 222 (2007) 536-555.

[20] A. Guittet, M. Theillard, F. Gibou, A stable projection method for the incompressible NavierStokes equations on arbitrary geometries and adaptive Quad/Octrees, J. Comput. Phys. 292 (2015) 215-238.

[21] A. Guittet, M. Lepilliez, S. Tanguy, F. Gibou, Solving elliptic problems with discontinuities on irregular domains - the Voronoi Interface Method, J. Comput. Phys. 298 (2015) 747-765.

[22] T.A. Johnson and V.C. Patel, Flow past a sphere up to a reynolds number of 300, Journal of Fluids Mechanics, 378 (1999) 19-70.

[23] M. Kang, R. Fedkiw, X.-D. Liu, A boundary condition capturing method for multiphase incompressible flow, J. Sci. Comput. 15 (2000) 323-360.

[24] S. Kang, G. Iaccarino, F. Ham, P. Moin, Prediction of wall-pressure fluctuation in turbulent flows with an immersed boundary method, J. Comput. Phys 228 (2009) 6753-6772.

[25] B. Lalanne, L. Rueda Villegas, S. Tanguy, F. Risso, On the computation of viscous terms for incompressible two-phase flows with Level Set/Ghost-Fluid Method, J. Comput. Phys. 301 (2015) 289-307.

[26] R.J. Leveque, Z. Li, Immersed interface methods for Stokes flow with elastic boundaries or surface tension, SIAM J. Sci. Comput. 18 (1997) 709-735.

[27] X.-D. Liu, R. Fedkiw, M. Kang, A boundary condition capturing method for Poissons equation on irregular domains, J. Comput. Phys. 160 (2000) 151-178.

[28] H. Liu, S. Krishnan, S. Marella, H.S. Udaykumar, Sharp interface Cartesian grid method II: A technique for simulating droplet interactions with surfaces of arbitrary shape, J. Comput. Phys. 210 (2005) 32-54.

[29] J.Magnaudet, M. Rivero, J. Fabre, Accelerated flows past a rigid sphere or a spherical bubble. Part 1. Steady straining flow. J. Fluid Mech. 284 (1995) 97-135.

[30] S. Marella, S. Krishnan, H. Liu, H.S. Udaykumar, Sharp interface Cartesian grid method I: An easily implemented technique for $3 \mathrm{D}$ moving boundary computations, J. Comput. Phys. 210 (2005) 1-31.

[31] T. Menard, S. Tanguy, A. Berlemont, Coupling level set/VOF/Ghost-Fluid methods: Validation and application to 3D simulation of the primary break-up of a liquid jet, Int. J. of Multiphase Flow 33 (2007) 510-524.

[32] R. Mittal and G. Iaccarino. Immersed boudary methods. Annu. Rev. Fluid. Mech, (2005) 239-261. 
[33] R. Mittal, H. Dong, M. Bozkurttas, F.M. Najjar, A. Vargas, and A. von Loebbecke. A versatile sharp interface immersed boundary method for incompressible flows with complex boundaries, J. Comput. Phys. 227 (2008) 4825-4852.

[34] Y.T. Ng, C. Min, F. Gibou, An efficient fluidsolid coupling algorithm for single-phase flows, J. Comput. Phys. 228 (2009) 8807-8829.

[35] E. Noel, A. Berlemont, J. Cousin, T. Menard, Application of the immersed boundary method to simulate flows inside and outside the nozzles, ICLASS 2012, $12^{\text {th }}$ Triennial International Conference on Liquid Atomization and Spray Systems, Heidelberg, Germany, September 2-6, 2012

[36] S. Osher, J.A. Sethian, Fronts propagating with curvature-dependent speed: algorithms based on HamiltonJacobi formulations, J. Comput. Phys. 79 (1988) 12-49.

[37] C.S. Peskin, Flow patterns around heart valves: A numerical method, J. Comput. Phys. 10 (1977) 252-271.

[38] S. Popinet, S. Zaleski, A front-tracking algorithm for accurate representation of surface tension, Int. J. Numer. Methods Fluids 30 (1999) 775-793.

[39] N. Rasmussen, D. Enright, D. Nguyen, S. Marino, N. Geiger, S. Hoon and R. Fedkiw, Directible Photorealistic Liquids, ACM SIGGRAPH/ Eurographics Symposium on Computer Animation (SCA), edited by R. Boulic and D. Pai (2004) pp 193-202.

[40] F.W. Roos and W.W. Willmarth, Some experimental results on sphere and disk drag, AIAA J., 9 (1971) 285-291.

[41] D. Rossinelli, B. Hejazialhosseini, W. Van Rees, M. Gazzola, M. Bergdorf, P. Koumoutsakos, MRAG-I2D: Multi-resolution adapted grids for remeshed vortex methods on multicore architectures, J. Comput. Phys. 288 (2015) 1-18.

[42] R. Scardovelli, S. Zaleski, Direct numerical simulation of free-surface and interfacial flow, Annu. Rev. Fluid Mech. 31 (1999) 567-603.

[43] S. Sikalo,H.-D. Wilhelm, I.V. Roisman, S Jakirlic, C. Tropea, Dynamic contact angle of spreading droplets: Experiments and simulations, Physics of Fluids, 17 (205) 62103-62116.

[44] J.H. Snoeijer, B. Andreotti, Moving Contact Lines: Scales, Regimes, and Dynamical Transitions, Annual Review of Fluid Mechanics 45 (2013) 269-292.

[45] P. D.M. Spelt, A level-set approach for simulations of flows with multiple moving contact lines with hysteresis, J. Comput. Phys. 207 (2005) 389-404.

[46] Y Sui, H Ding, P.D.M. Spelt, Numerical simulations of flows with moving contact lines, Annual Review of Fluid Mechanics 46 (2014) 97-119.

[47] M. Sussman, P. Smereka, S. Osher, A Level Set approach for computing solutions to incompressible two-phase flow, J. Comput. Phys. 114 (1994) 146-159.

[48] M. Sussman, K.M. Smith, M.Y. Hussaini, M. Ohta, R. Zhi-Wei, A sharp interface method for incompressible two-phase flows, J. Comput. Phys. 221 (2007) 469-505. 
[49] S. Taneda, Experimental investigation of the wake behind a sphere at low Reynolds numbers, J. Phys. Soc. Japan 11 (1956) 1104-1108.

[50] S. Tanguy, T. Menard, A. Berlemont, A Level-Set Method for vaporizing two-phase flows, J. Comput. Phys. 221 (2007) 837-853.

[51] S. Tanguy, M. Sagan, B. Lalanne, F. Couderc, C. Colin, Benchmarks and numerical methods for the simulation of boiling flows, J. Comput. Phys. 264 (2014) 1-22.

[52] G. Tryggvason, B. Bunner, A. Esmaeeli, D. Juric, N. Al-Rawahi, W. Tauber, J. Han, S. Nas, Y.-J. Jan, A Front-Tracking Method for the Computation of Multiphase Flow, J. Comput. Phys. 169 (2001) 708-759.

[53] D.J. Tritton, Physical Fluid Dynamics, Oxford Science Publications, 1988.

[54] Y. Tseng, J. H. Ferziger, A ghost-cell immersed boundary method for flow in complex geometry, J. Comput. Phys. 192 (2003) 593-623.

[55] M. Uhlmann, An immersed boundary method with direct forcing for the simulation of particulate flows, J. Comput. Phys. 209 (2005) 448-476.

[56] S. Vincent, A. Sarthou, J.P. Caltagirone, F. Sonilhac, P. Fevrier, C. Mignot, G. Pianet, Augmented Lagrangian and penalty methods for the simulation of two-phase flows interacting with moving solids. Application to hydroplaning flows interacting with real tire tread patterns, J. Comput. Phys. 230 (2011) 956-983.

[57] S. Vincent, J.C. Brandle de Motta, A. Sarthou, J.L. Estivalezes, O. Simonin, E. Climent, A lagrangian VOF tensorial penalty method for the DNS of resolved particle-laden flows, J. Comput. Phys. 256 (2014) 582-614.

[58] W. Xie and P.E. Desjardin, A level set embedded interface method for conjugate heat transfer simulations of low speed 2D flow, Computers \& Fluids, 37 (2008) 1262-1275.

[59] S. Xu, Z.J. Wang, An immersed interface method for simulating the interaction of a fluid with moving boundaries, J. Comput. Phys. 216 (2) (2006) 454493.

[60] S. Xu, The immersed interface method for simulating prescribed motion of rigid objects in an incompressible viscous flow, J. Comput. Phys. 227 (2008) 5045-5071.

[61] J. Yang, E. Balaras, An embedded-boundary formulation for large-eddy simulation of turbulent flows interacting with moving boundaries,J. Comput. Phys. 215 (2006) 12-40.

[62] T. Ye, R. Mittal, H.S. Udaykumar, and W. Shyy, An accurate cartesien grid method for viscous incompressible flows with complex immersed boundaries, J. Comput. Phys. 156 (1999) 209-240.

[63] K. Yokoi, F. Xiao, H. Liu, K. Fukasaku, Three-dimensional numerical simulation of flows with complex geometries in a regular Cartesian grid and its application to blood flow in cerebral artery with multiple aneurysms, J. Comput. Phys. 202 (2005) 1-19.

[64] L. Zhilin, An overview of the immersed interface method and its applications, Taiwanese Journal of Mathematics, 7 (2003) 1-49. 